\title{
Topics in Proton Structure: BSM Answers to its Radius Puzzle and Lattice Subtleties within its Momentum Distribution
}

Michael Chaim Freid

William \& Mary - Arts \& Sciences, mcfreid@gmail.com

Follow this and additional works at: https://scholarworks.wm.edu/etd

Part of the Physics Commons

\section{Recommended Citation}

Freid, Michael Chaim, "Topics in Proton Structure: BSM Answers to its Radius Puzzle and Lattice Subtleties within its Momentum Distribution" (2019). Dissertations, Theses, and Masters Projects. Paper 1563898994.

http://dx.doi.org/10.21220/s2-vs74-nv62

This Dissertation is brought to you for free and open access by the Theses, Dissertations, \& Master Projects at W\&M ScholarWorks. It has been accepted for inclusion in Dissertations, Theses, and Masters Projects by an authorized administrator of W\&M ScholarWorks. For more information, please contact scholarworks@wm.edu. 
Topics in Proton Structure: BSM answers to its radius puzzle and lattice subtleties within its momentum distribution

\author{
Michael Chaim Freid \\ Williamsburg, VA
}

MS Physics, College of William \& Mary, 2013

BS Physics and Computer Science, Rensselaer Polytechnic Institute, 2011

A Dissertation presented to the Graduate Faculty of The College of William \& Mary in Candidacy for the Degree of Doctor of Philosophy

Department of Physics

College of William \& Mary

May 2019 
(C) 2019

Michael Chaim Freid

All Rights Reserved 


\section{APPROVAL PAGE}

This dissertation is submitted in partial fulfillment of the requirements for the degree of
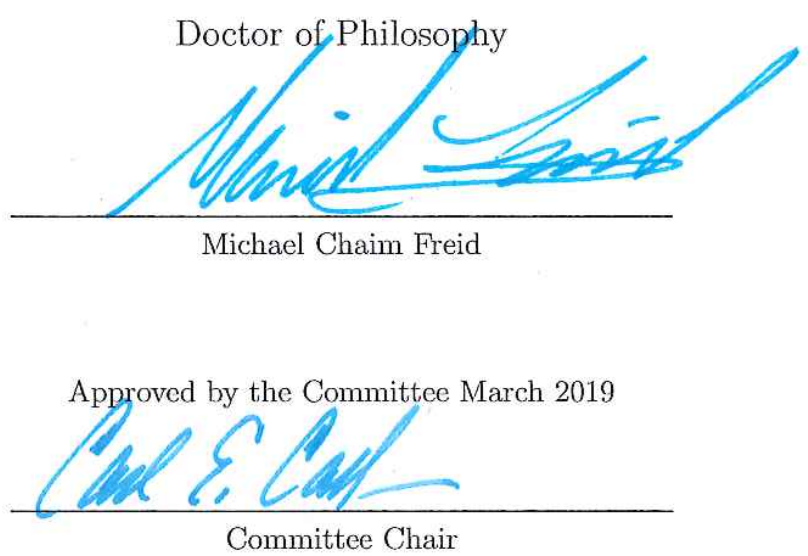

Professor Carl Carlson, Physics

College of William \& Mary

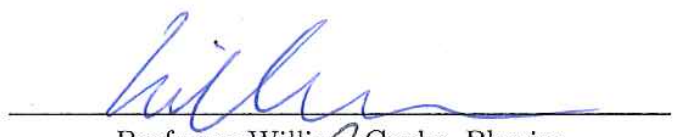

Professor-Willian Cooke, Physics

Cohlege of William \& Mary

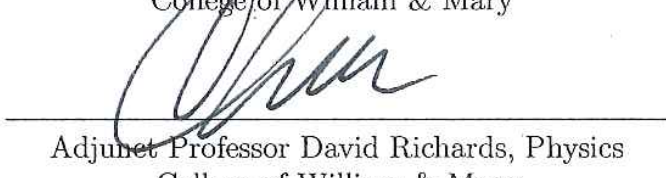

College of William \& Mary

Senior Staff Scientist, Thomas Jefferson National Accelerator Facility
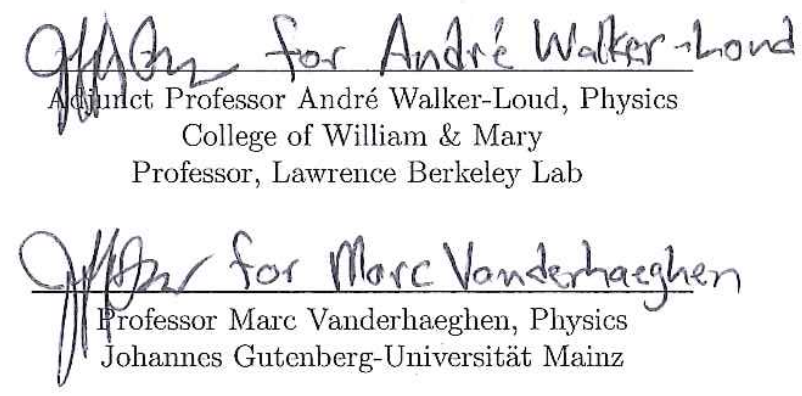


\begin{abstract}
This thesis covers two subjects, briefly outlined below, of hadronic physics.

The proton radius puzzle and the muon anomalous magnetic moment discrepancy point to possible signs of lepton-universality violation. We introduce the context and background necessary to understand these two problems. And we analyze two suitable beyond-the-standard-model solutions, one vector-based and one scalar-based, which simultaneously solves both issues. Furthermore, we demonstrate that the parameter space of the respective solutions can be chosen so as to not be completely forbidden by considered experimental constraints. Specifically, we show that certain violations, analyzed by other authors in the context of similar solutions to those proposed, are ameliorated if one adds to the proposed solutions particular criteria required for renormalizability.

We also explore the field of lattice perturbation theory in the context of deep inelastic scattering. In particular, we study the connection between lattice-based Euclidean parton distributions and their experiment-based Minkowskian equivalents. These parton distributions are extracted from deep inelastic scattering processes in which leptons are scattered of hadronic targets at energy levels high enough to resolve the internal hadronic structure. To synchronize experiment with theory, one must find the proper matching criteria to transform approximations of parton distributions, known as quasidistributions, as they are resolved on a computer (i.e. the lattice), back to the full parton distributions extracted from experiment. We provide the necessary background to understand context of the above and show the procedures necessary to match experiment with the lattice. We demonstrate how one must tread carefully to properly perform such a matching.
\end{abstract}




\section{Table of Contents}

Acknowledgements $\quad$ iii

Dedication $\quad$ iv

List of Figures $\quad$ v

1 Sectors of the Standard Model, and beyond 1

1.1 The electroweak sector . . . . . . . . . . . . . . 2

1.2 The Strong Sector . . . . . . . . . . . . . . . . . . . . . 13

2 The Proton Radius Puzzle 19

2.1 The details of the puzzle . . . . . . . . . . . . . . 22

2.2 A renormalizable vector theory . . . . . . . . . . . . . 26

2.3 Scalar Theory . . . . . . . . . . . . . . . . . . . 37

2.4 Conclusion . . . . . . . . . . . . . . . . . . . . 42

3 Subtleties within the Euclidean Quark Quasidistribution 45

3.1 Introduction to DIS and the quark distribution function . . . . . . 47

3.2 The matching process and an introduction to LPT . . . . . . . 57

3.3 The One-Loop Corrections . . . . . . . . . . . . . . . . . 62

3.4 Poles in the Euclidean Integrals $\ldots \ldots$. . . . . . . . . . . 69

3.5 The Lattice vs. Lattice Perturbation Theory . . . . . . . . . . . . . 71

4 Concluding discussions 76

4.1 Discussions on the proton radius puzzle . . . . . . . . . . . 77 
4.2 Discussions on lattice quark quasidistribution $\ldots \ldots . \ldots 79$

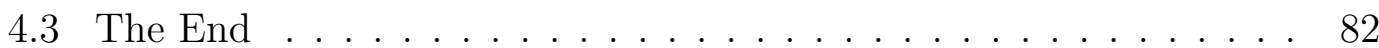

$\begin{array}{lr}\text { Bibliography } & 83\end{array}$

$\begin{array}{ll}\text { Vita } & 87\end{array}$ 


\section{Acknowledgements}

This thesis would not exist if I were not engulfed by overwhelming support. I owe my sincerest thanks to my advisor, Carl Carlson, for his patience, his illuminating intellect, and his storytelling. And to Andre Walker-Loud, for his belief in me when I needed it most. To Marc Sher, who taught me what it means to be a pen \& paper physicist. And to Chris Carone, for not lowering the bar to a common denominator, and thus raising the bar for us all.

I must mention my professor and dear friend Jim Napolitano, who personally went out of his way to assure my future in physics. And my many thanks to David Richards, for first accepting me into the theory scholarship program at Jefferson Lab and for continuing to support me through the years.

Of course, my office must be noted: The Daily Grind. Thank you to Scott for the personality he infused into a coffeehouse, and the characters he found to enliven it.

And I cannot forget Victoria Owen for building an oasis. 
Dedicated to our secretaries, whose personalities fill the room. 


\section{List of Figures}

1.1 1-dimensional cross-section of the Higgs potential, with the location of the non-zero VEV shown explicitly. . . . . . . . . . . . .

1.2 The Gell-Mann Goldberger Kroll Low (GGKL) process $\nu \bar{\nu} \rightarrow W^{+} W^{-} 11$

1.3 Freely propogating quark . . . . . . . . . . . . . . 15

1.4 Gluon-loop corrections to the quark propagator . . . . . . . . . . 15

1.5 Gluonic Self Interactions . . . . . . . . . . . . . . . . . . . 16

2.1 Rutherford Scattering: an incoming electron with energy E elastically scatters off a point-like proton target with scattering angle 

2.2 1st order contribution to releativistic electron-proton scattering, the result of which is the Rosenbluth formula. An electron with incoming momentum $l$, and outgoing momentum $l^{\prime}$, scatters off a proton with incoming momentum $p$, and outgoing momentum $p^{\prime}$. . . . .

2.3 The $\left(C_{V}, C_{A}, m_{\phi}\right)$ parameter space necessary to solve the proton radius puzzle and muonic $g-2$ discrepancy while satisfying the considered experimental constraints. The regions bordered be solid black lines refer to constraints on $C_{V}^{\mu}$. The band bordered by dashed lines refer to constraints on $C_{A}^{\mu}$. The green band, outlined by solid black lines, is the region of $\left(C_{V}^{\mu}, m_{\phi}\right)$ required to solve the proton radius problem $( \pm 2 \sigma)$. The shaded red region, bordered below by the black line, is the excluded region of $\left(C_{V}^{\mu}, m_{\phi}\right)$ due to the constraint that the branching ratio for $W$ goes to $\mu \nu \phi_{V}+\mu \nu \phi_{A}$ must be less than $4 \%$ (under the assumption that $C_{A}^{\mu}$ is set to solve the muonic $g-2$ problem). The shaded orange region is the restricted region on $\left(C_{V}^{\mu}, m_{\phi}\right)$ due to energy splittings in muonic $\mathrm{Mg}$ and $\mathrm{Si}$ at $2 \sigma$. The green band, outlined by dashed lines, is the desired region of $\left(C_{A}^{\mu}, m_{\phi}\right)$ which solves solve the muonic $g-2$ problem $( \pm 2 \sigma)$ (under the assumption that $C_{V}^{\mu}$ is set to solve the proton radius problem $( \pm 2 \sigma)) \ldots \ldots \ldots \ldots \ldots \ldots \ldots \ldots$

2.4 Lowest-order contributions to the muon's anamlous magnetic moment from QED, weak, and hadronic interactions respectively. . . .

2.5 BSM contributions to the muon's anomalous magnetic moment. Here $\phi$ repeseents the addition of both vector and axial-vector diagrams. . . . . . . . . . . . . . . . . . . . 
2.6 BSM contributions to $W$-decay $W \rightarrow \mu \nu \phi$, including the shadow $W$ branch. . . . . . . . . . . . . . . . . . 33

2.7 BSM contributions to Z-decay: $Z \rightarrow \mu^{-} \mu^{+} \phi \ldots \ldots \ldots$

2.8 BSM contributions to the muon's anomalous magnetic moment. Here $\phi$ repeseents the addition of both vector and axial-vector diagrams. . . . . . . . . . . . . . . . . .

2.9 The $\left(C_{S}, C_{P}, m_{S}\right)$ parameter space necessary to solve the proton radius puzzle and muonic $g-2$ discrepancy while satisfying the considered experimental constraints. The regions bordered be solid black lines refer to constraints on $C_{S}^{\mu}$. The dashed lines represent constraints on $C_{P}^{\mu}$. The green band, outlined by solid black lines, is the region of $\left(C_{S}^{\mu}, m_{S}\right)$ required to solve the proton radius problem $( \pm 2 \sigma)$. The shaded red region, bordered below by the black line, is the excluded region of $\left(C_{S}^{\mu}, m_{S}\right)$ due to the constraint that the branching ratio for $W$ goes to $\mu \nu \phi_{S}+\mu \nu \phi_{P}$ must be less than $4 \%$ (under the assumption that $C_{P}^{\mu}$ is set to solve the muonic $g-2$ problem). The shaded orange region is the restricted region on $\left(C_{S}^{\mu}, m_{S}\right)$ due to energy splittings in muonic $\mathrm{Mg}$ and $\mathrm{Si}$ at $2 \sigma$. The dashed green line is the desired parameter space of $\left(C_{P}^{\mu}, m_{S}\right)$ which solves solve the muonic $g-2$ problem (under the assumption that $C_{S}^{\mu}$ is set to solve the proton radius problem). . . . . . . .

2.10 Scalar BSM contribution to the $W$-decay $W \rightarrow \mu \nu \phi_{S}+\mu \nu \phi_{P}$. In the figure, $\phi$ represents the addition of the scalar and pseudoscalar contributions. . . . . . . . . . . . . . . . .

3.1 Deep Inelastic Scattering (DIS): a lepton with momentum $l$ is scattered off a proton with momentum $p$ resulting in a shower of hadronic states $X$. The inelasticity of DIS results from a sufficient energy transfer $q$, such that the lepton interacts with an individual quark of momentum $k$. In light-cone coordinates $k$ is a fraction of the proton's momentum $p \ldots \ldots \ldots \ldots \ldots \ldots$

3.2 In the infinite momentum frame, the proton moving with very large momentum in the $z$ direcion becomes Lorentz contracted and time dilated. The constituent quarks and gluons become spread out and frozen. The photon probe with momentum $q$ also moving in he $z$ direction interacts with a free quark unaffected by low-energy bound-state interactions. . . . . . . . . . . . . .

3.3 An on-shell, zero mass, free constituent quark of momentum $k$ moving in the $z$ direction scatters off photon with momentum $q$ also moving in the $z$ direction, resulting in a quark of momentum $k^{\prime}$ with $k_{z}=-k_{z}^{\prime} \ldots \ldots \ldots \ldots \ldots \ldots \ldots$ 
3.4 The lightcone coordinates analog of Fig. 3.3. An on-shell, zero mass, free constituent quark of momentum $k$ moving in the + direction scatters off photon with momentum $q$, resulting in a quark of momentum $k^{\prime}$ moving in the - direction such that $k^{+}=k^{\prime-} \ldots \ldots$.

3.5 The one-loop corrections to the quark distribution function. Immediately, one notes that diagrams (c) and (d) can be gauged away by choosing light-front (or Axial in the case of the quasidistribution) gauge. Diagram (a) is the vertex correction. And diagram (b) is the wave-function renormalization. . . . . . . . . . . . .

3.6 The transverse momentum integration region containing the UV divergence in the one-loop vertex correction on the lattice. We can extract the form of the UV divergence by integrating in polar coordinates. The lost terms from the corners will be small for small $a$. Note that the circle inscribed by the rectangle shown is not to scale. To capture the UV divergence correctly, one can show that the circle's radius is $n / a>\pi / a$ where $n=3.17591 \ldots \ldots$. . .

3.7 The vertex correction to the quark quasidistribution, normalized by $\alpha_{s} C_{F} /(2 \pi)$, calculated in Minkowski space (blue dashed line) versus the same calculated via LPT in Euclidean space (orange solid line) to leading order in $\mathrm{m}^{2} / p_{z}^{2}$ for momentum fraction $x$ between 0 and 1. Here $p_{z}$ is $2 \mathrm{GeV}$, and the quark mass $m$ is $0.02 \mathrm{GeV}$. $\Lambda \leftrightarrow n / a$ is $2 \mathrm{GeV}$. When $\Lambda<p_{z}$ the sign of the $x \rightarrow 1$ pole flips for the LPT (orange solid line) result while the Minkoski result remains qualitatively unaffected. The red bar denotes the region where $x=m / p_{z}$. To the left of this line, the expansion in $m^{2} / p_{z}^{2}$ is not valid.

3.8 Locations of the $k^{0}$ poles within the Minkowskian integral $I_{1 M}$. Crosses represent the two poles arising from the quark propagator, and circles represent the two poles from the gluon propagator. The relative locations of each pole with respect to one another is of importance and is shown accurately within this plot. The vertical dashed line demonstrates a given location of the Wick rotation

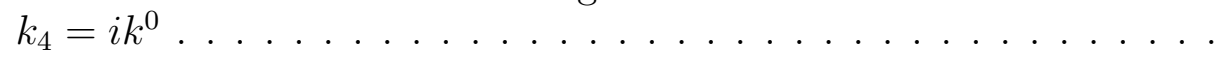


The limits of my language mean the limits of my world. Ludwig Wittgenstein 


\section{Chapter 1}

\section{Sectors of the Standard Model, and beyond}

nenomena which occur under certain conditions, will recur in the same man-
mentalists collaborate to uncover nature's inner workings. The work herein will be twofold. One, analyze and consider additions to the theoretical framework that predicts, possibly incompletely, certain observed phenomena from a given set of conditions. Two, properly understand and control the assumptions embedded within theory so that we may correctly calculate its predictions.

This is no trivial task and we build upon the works of giants. The current mountain of knowledge regarding nature, as seen through the lens of modern physical experiment, can be summed up in what we now call the "standard model."

The standard model encompasses the strong interactions and quarks, which form the bound states of baryons, mesons, and nuclei; and the electroweak interactions and leptons which are responsible for the bound states of our atoms, the radioactive decay of heavy nuclei, and in a more practical sense, what we perceive as "solid" and "visible." For convenience, we ignore gravity.

Beyond-standard-model (BSM) physics is the extension of our knowledge beyond the standard model. Therefore, before we begin this extension of knowledge, we must first sufficiently understand that which the standard model already predicts.

The standard model is built upon least-action-principle assumption. That is, the matter of the considered theory will interact in such as a way as to minimize a defined quantity we call the "action"

$$
S=\int d^{4} x \mathcal{L}\left(\psi, \partial_{\mu} \psi\right)
$$


where infinitesimal changes to the fields $\psi$ leave the action unchanged. That is,

$$
\delta S=0
$$

The least-action-principle accounts for the kinematic motion of fields through spacetime and for the dynamics of interacting fields. An interested reader should refer to [1].

Of course, so far, all we have done in regards to defining the details of the theory is move the burden onto defining the Lagrangian $\mathcal{L}$.

The Lagrangian is a function of quantum mechanical fields, which operate on a Hilbert space to create and destroy particles, and which satisfy translation, rotation, and Lorentz symmetry in addition to certain local gauge symmetries which will account for the "interactive" nature of matter.

We will study the standard model through the lens of local gauge symmetries - that is the gauge-group of transforming fields which leave the theory (i.e., the action, and by proxy the Lagrangian - up to a constant) unchanged. In section 1.1, we study the foundations of the electroweak $\left(\mathrm{SU}(2)_{L} \times \mathrm{U}(1)\right)$ gauge group as it is this sector of the standard model which we wish to extend first in chapter 2 . In section 1.2 , we will study the strong interaction $\mathrm{SU}(3)$ to build-up to a particular use case in chapter 3.

\subsection{The electroweak sector}

Electroweak theory is the study of interacting fermion (spin-half) and boson (integer spin) fields which covariantly transform under the local gauge symmetry $\left(\mathrm{SU}(2)_{L} \mathrm{x}\right.$ $\mathrm{U}(1)$ ) such that the action of the theory is left undisturbed.

All the dynamics of electromagnetism (i.e. Maxwell's equations) can be accounted for by building a $\mathrm{U}(1)$ scalar, denoted $B_{\mu \nu} B^{\mu \nu}$, made up of interacting $\mathrm{U}(1)$ fields $B_{\mu}$, placing it into the Lagrangian, and applying the consequences of the least-actionprinciple. The tensor $B_{\mu \nu}$ we call the "field stength tensor" and define it as

$$
B_{\mu \nu} \equiv \partial_{\mu} B_{\nu}-\partial_{\nu} B_{\mu}
$$

We now have the basis for a theory which predicts light-like behavior. On its own, fields which behave like electromagnetism cannot predict notable subatomic phenomena such as nuclear fission.

To account for radioactive beta decay, we consider fields which transform under $\mathrm{SU}(2)$ and include in the Lagrangian their corresponding field strength tensor

$$
W_{\mu \nu}^{i} \equiv \partial_{\mu} W_{\nu}^{i}-\partial_{\nu} W_{\mu}^{i}+g \epsilon^{i j k} W_{\mu}^{j} W_{\nu}^{k}
$$


where $i$ runs from one to three. As standard, repeated indices are contracted. Here, $g$ is the coupling strength of $\mathrm{SU}(2)$.

The $\mathrm{U}(1)$ and $\mathrm{SU}(2)$ fields propagate through free space and interact amongst themselves within the kinetic piece of the Lagrangian,

$$
\mathcal{L}_{\text {Kinet. }}=-\frac{1}{4} B_{\mu \nu} B^{\mu \nu}-\frac{1}{4} W_{\mu \nu}^{i} W_{i}^{\mu \nu}
$$

such that $\mathcal{L}_{\text {Kinet. }}$ is invariant under local gauge transformations

$$
\begin{aligned}
B_{\mu} & \rightarrow B_{\mu}+\frac{1}{g^{\prime}} \partial_{\mu} \beta(x) \\
\frac{\sigma_{i}}{2} W_{\mu}^{i} & \rightarrow U(x) \frac{\sigma_{i}}{2} W_{\mu}^{i} U^{\dagger}(x)-\frac{i}{g}\left(\partial_{\mu} U(x)\right) U^{\dagger}(x)
\end{aligned}
$$

where $\beta(x)$ is an arbitrary scalar function of position with $g^{\prime}$ being the coupling strength of the $\mathrm{U}(1)$ field, and where $g$ is the coupling strength of the $\mathrm{SU}(2)$ fields with $\sigma_{i}$ denoting the three Pauli matrices. And where the $\mathrm{SU}(2)$ transformation is

$$
U(x) \equiv \exp \left\{i \frac{\sigma^{i}}{2} \alpha_{i}(x)\right\}
$$

with $\alpha_{i}$ denoting three arbitrary scalar functions of position in space-time.

We now add matter in the form of fermions, denoted $\psi$, where $\psi$ is a 4 -component Dirac spinor with $\frac{1}{2}$ spin angular momentum. That is,

$$
S^{2} \psi=\left(\frac{1}{2}\right)^{2} \psi
$$

where $\vec{S}$ is the spin operator

$$
\vec{S}=\left(\begin{array}{cc}
\vec{\sigma} / 2 & 0 \\
0 & \vec{\sigma} / 2
\end{array}\right)
$$

As spin one-half fields, the fermions can be naturally split into right and left-handed forms via the chiral projection operators

$$
\begin{aligned}
& P_{R}=\frac{1}{2}\left(1+\gamma_{5}\right) \\
& P_{L}=\frac{1}{2}\left(1-\gamma_{5}\right),
\end{aligned}
$$

where

$$
\gamma_{5}=i \gamma_{0} \gamma_{1} \gamma_{2} \gamma_{3}
$$

such that we can write the right-handed and left-handed fermions respectively as

$$
\begin{aligned}
& \psi_{R}=P_{R} \psi \\
& \psi_{L}=P_{L} \psi
\end{aligned}
$$


In nature, we observe only the left-handed fermions coupling to the $\mathrm{SU}(2)$ fields as $\mathrm{SU}(2)$ doublets. With interactions, the electroweak Lagrangian is then

$$
\begin{aligned}
\mathcal{L}_{\mathrm{EW}} & =i \bar{\psi}_{L} \not D_{\mathrm{L}} \psi_{L}+i \bar{\psi}_{R}^{a} \not D_{\mathrm{R}} \psi_{R}^{a} \\
& -\frac{1}{4} B_{\mu \nu} B^{\mu \nu}-\frac{1}{4} W_{\mu \nu}^{i} W_{i}^{\mu \nu}
\end{aligned}
$$

where the covariant derivatives for the left-handed and right-handed fields respectively are

$$
\begin{aligned}
& \not D_{\mathrm{L}}=\gamma^{\mu}\left[\partial_{\mu}+i g \frac{\sigma_{i}}{2} W_{\mu}^{i}+i g^{\prime} \frac{Y}{2} B_{\mu}\right] \\
& \not D_{\mathrm{R}}=\gamma^{\mu}\left[\partial_{\mu}+i g^{\prime} \frac{Y}{2} B_{\mu}\right],
\end{aligned}
$$

and

$$
\bar{\psi} \equiv \psi^{\dagger} \gamma^{0}
$$

The index $a$ on the right-handed fields runs from one to two and corresponds with the two fermions encapsulated within the left-handed doublet. It is understood that we implicitly sum over all generations of fermions in $\mathcal{L}_{\mathrm{EW}}$. For instance, if we are interested in the 1st generation of leptons, then

$$
\begin{aligned}
& \psi_{L}=\left(\begin{array}{c}
\nu_{e} \\
e
\end{array}\right)_{L} \\
& \psi_{R}^{1}=\nu_{e, R} \\
& \psi_{R}^{2}=e_{R},
\end{aligned}
$$

where we've grouped the left-handed fields, such as the electron and electron-neutrion, into a 2-component spinor (with 8 components total), and left the right-handed fields as standalone 4-component spinors.

To maintain the invariance of the Lagrangian under the local $\mathrm{SU}(2)_{L}$ transformations of $W$ and the local $\mathrm{U}(1)$ transformations of $B$, the fermion fields must correspondingly transform as

$$
\begin{aligned}
& \psi_{L} \rightarrow e^{i Y \beta(x)} U(x) \psi_{L} \\
& \psi_{R} \rightarrow e^{i Y \beta(x)} \psi_{R} .
\end{aligned}
$$

Note that because the $\mathrm{U}(1)$ fields $B_{\mu}$ do not couple to themselves, we have additional freedom in the choice of the hypercharge $Y$ for each fermion $\psi$ whereas the weak charge for each fermion is fixed by $\frac{\sigma_{i}}{2}$. 
Though we now have a complete $\mathrm{SU}(2)_{L} \mathrm{x} U(1)$ theory, we are missing one essential ingredient to have the theory in order to correspond with reality: mass. Directly adding in a fermion mass term such as $m \bar{\psi} \psi$ into the Lagrangian mixes the left-handed and right-handed fields and breaks its gauge invariance as the left-handed fields transform under $S U(2)$ while the right-handed fields do not.

To see this explicitly, consider an fermion mass term of the form $m_{\psi} \bar{\psi} \psi$. From (1.13), we can rewrite the mass term as

$$
\begin{aligned}
m_{\psi} \bar{\psi} \psi & =m_{\psi}\left[\frac{1}{4} \bar{\psi}\left(1+\gamma_{5}\right)\left(1+\gamma_{5}\right) \psi+\frac{1}{4} \bar{\psi}\left(1-\gamma_{5}\right)\left(1-\gamma_{5}\right) \psi\right] \\
& =m_{\psi}\left[\bar{\psi}_{L} \psi_{R}+\bar{\psi}_{R} \psi_{L}\right]
\end{aligned}
$$

where we have used the $\gamma_{5}$ identities

$$
\begin{array}{r}
\gamma_{5}^{2}=1 \\
\left\{\gamma_{5}, \gamma_{\mu}\right\}=0
\end{array}
$$

and noted that the left-handed (right-handed) anti-fields $\bar{\psi}_{\mathrm{L} / \mathrm{R}}$ are obtained by taking the transpose complex-conjugate of their respective regular fields, after being operated on by the left-handed (right-handed) projection operators. That is,

$$
\begin{aligned}
\bar{\psi}_{L} & =\left(\frac{1}{2}\left(1-\gamma_{5}\right) \psi\right)^{\dagger} \gamma_{0} \\
& =\left(\psi^{\dagger} \frac{1}{2}\left(1-\gamma_{5}\right)\right) \gamma_{0} \\
& =\bar{\psi} \frac{1}{2}\left(1+\gamma_{5}\right) \\
& =\bar{\psi} P_{R}
\end{aligned}
$$

Glancing at (1.22), we now see that the two mass terms in (1.24) are not invariant under the local transformation rules (1.22). Similarly, boson mass terms such as $m_{B} B_{\mu} B^{\mu}$ and $m_{W} W^{\mu} W_{\mu}$ will also break gauge invariance under the fields respective local transformations (1.6).

To simultaneously solve both of these issues, we introduce the scalar isospin doublet boson known as the Higgs field, denoted $\phi$,

$$
\phi=\frac{1}{\sqrt{2}}\left(\begin{array}{c}
\phi_{1}+i \phi_{2} \\
\phi_{3}+i \phi_{4}
\end{array}\right)
$$

with 4 degrees of freedom given by the real fields $\phi_{i}$. Being an isospin doublet, the Higgs field transforms in a way analogous to the left-handed fermions.

$$
\phi \rightarrow U(x) \phi
$$


where, $U(x)$ is given in (1.8).

Furthermore, we choose the potential for the Higgs field such that there exists a non-zero vacuum expectation value (VEV). That is, the ground state of the Lagrangian occurs at a non-zero Higgs field value where the ground state of the Lagrangian is defined by the minimum-value of the Hamiltonian, where, for a constant field, the Hamiltonian is

$$
\left.\mathcal{H}\right|_{\text {Constant Fields }}=-\mathcal{L}
$$

The electroweak Lagrangian becomes $\mathcal{L}_{\mathrm{EW}} \rightarrow \mathcal{L}_{\mathrm{EW}}+\mathcal{L}_{\text {Higgs }}$ where

$$
\mathcal{L}_{\mathrm{Higgs}}=\left(D_{\mu} \phi\right)^{\dagger}\left(D^{\mu} \phi\right)-\mu^{2} \phi^{\dagger} \phi-\lambda\left(\phi^{\dagger} \phi\right)^{2},
$$

in which the first term is the Higg's kinetic term and the remaining two terms form the Higg's potential.

We choose $\mu^{2}<0$ such that there exists a non-zero minimum within $\mu^{2} \phi^{\dagger} \phi+\lambda\left(\phi^{\dagger} \phi\right)^{2}$. This potential is 4-dimensional and difficult to conceptualize. In order to understand the shape, consider a 1-dimensional cross-section,

$$
V\left(\phi_{1}\right)=\mu^{2} \phi_{1}^{2}+\lambda \phi_{1}^{4}
$$

which we plot in Figure. 1.1 


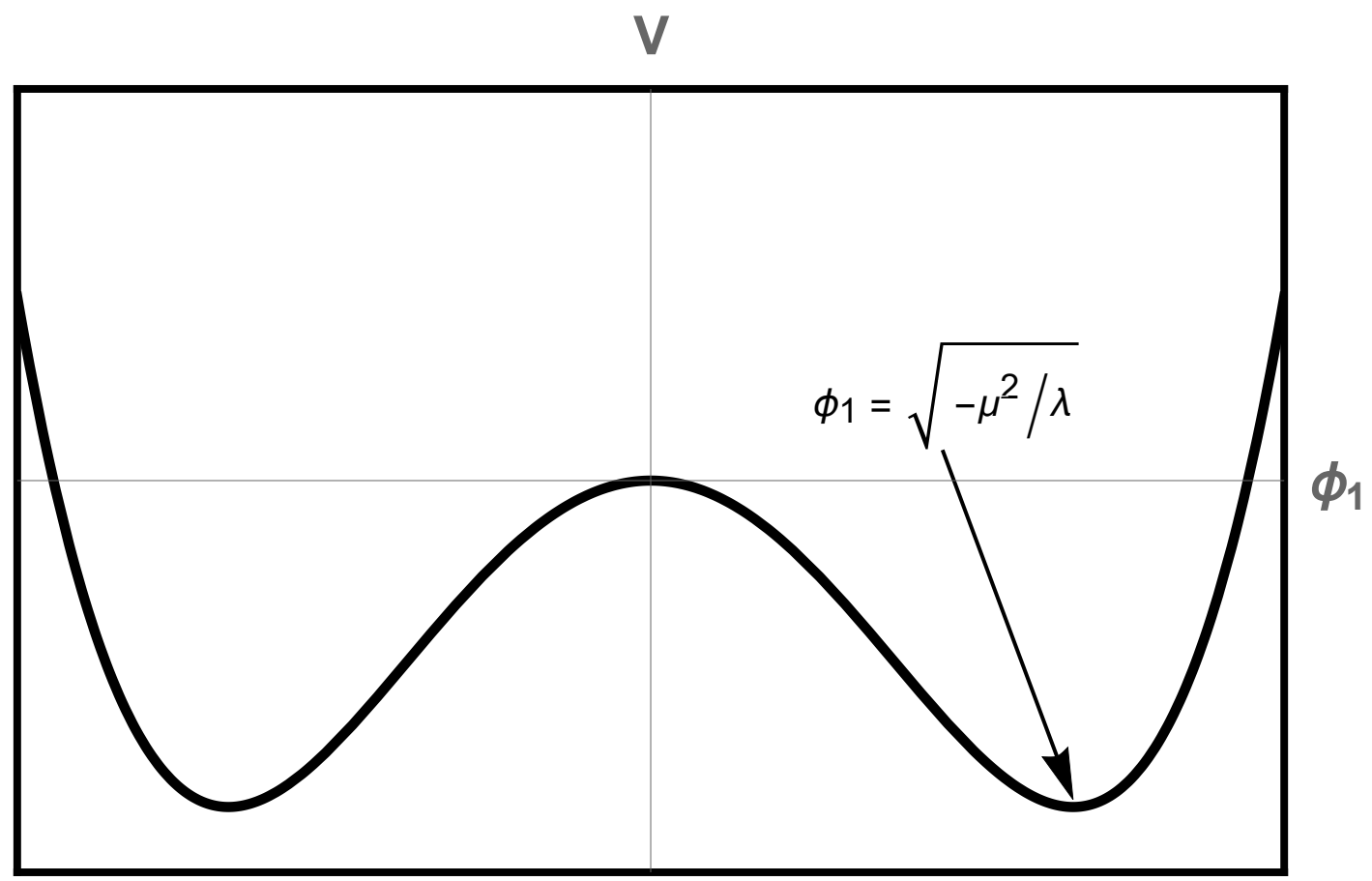

Figure 1.1: 1-dimensional cross-section of the Higgs potential, with the location of the non-zero VEV shown explicitly.

We shift $\phi$ such that its minimum potential energy occurs when the field is zero; thereby allowing us to freely apply perturbation theory as deviations from the Higg's minimum potential value. There are a continuous infinity of shifts within $\phi$ that accomplish this. To arrive at a definite result, we choose

$$
\phi \rightarrow \frac{1}{\sqrt{2}}\left(\begin{array}{c}
0 \\
\nu+h
\end{array}\right)
$$

where $\nu \equiv \sqrt{-\mu^{2} / \lambda}$, the VEV, is a real number and $h$ is the leftover real-valued Higgs field. Note that, by shifting $\phi$ around this VEV, 3 degrees of freedom

$$
\phi_{1}=\phi_{2}=\phi_{4} \rightarrow 0
$$

go to zero for the Higgs field that minimizes the potential given in (1.31). Though these degrees of freedom are zero in the static/chosen Higgs field, they have not disappeared from the Lagrangian. They will become manifest in the longitudinal polarization now available to the three gauge bosons which acquire mass (as shown below).

To see how this mass is acquired, and, in doing so, deriving the physical gauge boson fields, we take a careful look at the kinetic piece of the Higgs field $\left(D_{\mu} \phi\right)^{\dagger}\left(D^{\mu} \phi\right)$. Within this calculation, we will not be interested in the Higgs interactions and so we simply take $\phi \rightarrow \frac{1}{\sqrt{2}}\left(\begin{array}{l}0 \\ \nu\end{array}\right)$ in the steps below. 


$$
\begin{aligned}
\left.\left(D_{\mu} \phi\right)^{\dagger}\left(D^{\mu} \phi\right)\right|_{\phi=\left(\begin{array}{c}
0 \\
\nu
\end{array}\right)} & =\frac{1}{2}\left(\left[\partial_{\mu}-g \frac{\sigma_{i}}{2} W_{\mu}^{i}-\frac{g^{\prime} Y}{2} B_{\mu}\right] \phi\right)^{\dagger}\left[\partial_{\mu}-g \frac{\sigma_{i}}{2} W_{\mu}^{i}-\frac{g^{\prime} Y}{2} B_{\mu}\right] \phi \\
& =\left[\frac{i \nu}{\sqrt{8}}\left(\begin{array}{c}
g\left(W_{1, \mu}-i W_{2, \mu}\right) \\
-g W_{3, \mu}+g^{\prime} Y B_{\mu}
\end{array}\right)\right]^{\dagger}\left[\frac{i \nu}{\sqrt{8}}\left(\begin{array}{c}
g\left(W_{1}^{\mu}-i W_{2}^{\mu}\right) \\
-g W_{3}^{\mu}+g^{\prime} Y B^{\mu}
\end{array}\right)\right] \\
& =\frac{\nu^{2}}{8}\left[g^{2}\left(W_{1}^{2}+W_{2}^{2}\right)+\left(g^{\prime} Y B_{\mu}-g W_{3, \mu}\right)^{2}\right] \\
& =\frac{1}{2} M_{W}^{2} W^{+} W^{-}+\frac{1}{2} M_{Z}^{2} Z^{2}+0 \cdot A^{2}
\end{aligned}
$$

where, in the last step with the Higg's hypercharge $Y$ set to 1 , we have identified the mass eigenstates as

$$
\begin{aligned}
W^{ \pm} & =\frac{1}{\sqrt{2}}\left(W_{1} \mp i W_{2}\right) \\
Z & =\frac{1}{\sqrt{g^{2}+g^{\prime 2}}}\left(g W_{3}-g^{\prime} B\right) \\
A & =\frac{1}{\sqrt{g^{2}+g^{\prime 2}}}\left(g^{\prime} W_{3}+g B\right)
\end{aligned}
$$

with masses

$$
\begin{aligned}
M_{W} & =\frac{\nu g}{2} \\
M_{Z} & =\frac{\nu \sqrt{g^{2}+g^{\prime 2}}}{2} \\
M_{A} & =0
\end{aligned}
$$

such that we give the gauge fields a physical interpretation. As expected, three of the gauge bosons have acquired mass, while one, the photon $A$, remains massless. It will be useful to recognize the convenient parametrization of the coupling strengths $g$ and $g^{\prime}$ in terms of a mixing angle denoted $\theta_{W}$, that is

$$
\tan \theta_{W} \equiv \frac{g^{\prime}}{g}
$$

With this parameterization, we identify the mass eigenstates $Z$ and $A$ as rotations of the neutral $W^{3}$ and $B$ fields such that

$$
\begin{aligned}
& Z=\cos \theta_{W} W^{3}-\sin \theta_{W} B \\
& A=\sin \theta_{W} W^{3}+\cos \theta_{W} B .
\end{aligned}
$$

Furthermore, we see the amount of rotation determines the mass ratio between the charged $W^{ \pm}$fields and the neutral $Z$ field from

$$
\frac{M_{W}^{2}}{M_{Z}^{2}}=\cos ^{2} \theta_{W}
$$


As with the gauge bosons, we also wish to give masses to the fermion fields through a Higgs interaction. We accomplish this by adding to our Lagrangian the gauge-invariant Yukawa couplings

$$
-\lambda_{F}\left(\bar{\psi}_{L} \phi \psi_{R}+\text { h.c. }\right)
$$

where h.c. denotes the Hermitian conjugate of the previous term. Note that the addition of $\phi$ field, which transforms like an $\mathrm{SU}(2)$ doublet, fixes the gauge-invariance caused by the left-handed fermions in mass terms of the form $m_{\phi} \bar{\psi}_{L} \psi_{R}$.

To see how this coupling generates a fermion mass term, again we take $\phi \rightarrow \frac{1}{\sqrt{2}}\left(\begin{array}{l}0 \\ \nu\end{array}\right)$, such that the static piece of the Yukawa coupling for the first generation leptons becomes

$$
\begin{aligned}
-\lambda_{F} \frac{\nu}{\sqrt{2}}\left(\bar{e}_{L} e_{R}+\text { h.c. }\right) & =-\lambda_{F} \frac{\nu}{\sqrt{2}} \bar{e} e \\
& =-m_{e} \bar{e} e
\end{aligned}
$$

and

$$
m_{e} \equiv \lambda_{F} \frac{\nu}{\sqrt{2}}
$$

Note that the respective first generation neutrino remains massless.

Now that we have the physical masses for the fermions and gauge bosons, we must reevaluate their respective electroweak interactions in terms of the physical gauge fields to derive the electroweak Feynman rules. Consider again the covariant derivatives within the kinematic term of the fermion fields

$$
\begin{aligned}
& \not D_{\mathrm{L}}=\gamma^{\mu}\left[\partial_{\mu}+i g \frac{\sigma_{i}}{2} W_{\mu}^{i}+i \frac{g^{\prime} Y}{2} B_{\mu}\right] \\
& \not \mathrm{R}_{\mathrm{R}}=\gamma^{\mu}\left[\partial_{\mu}+i \frac{g^{\prime} Y}{2} B_{\mu}\right] .
\end{aligned}
$$

We must rewrite the gauge couplings in terms of the physical gauge bosons $W^{ \pm}, Z$, and $A$.

Let us begin with the neutral piece of the covariant derivative for the left-handed fields

$$
\begin{aligned}
i g \frac{\sigma_{3}}{2} W_{\mu}^{3}+\frac{i g^{\prime} Y}{2} B_{\mu} & =i g \frac{\sigma_{3}}{2}\left(\sin \theta_{W} A+\cos \theta_{W} Z\right)+\frac{i g^{\prime} Y}{2}\left(\cos \theta_{W} A-\sin \theta_{W} Z\right) \\
& =i\left(g \frac{\sigma_{3}}{2} \sin \theta_{W}+\frac{g^{\prime} Y}{2} \cos \theta_{W}\right) A+i\left(g \frac{\sigma_{3}}{2} \cos \theta_{W}-\frac{g^{\prime} Y}{2} \sin \theta_{W}\right) Z
\end{aligned}
$$

and similarly for the right-handed fields. 
Therefore, a given lepton $l$ (with hypercharge $Y=-1$ for the left-handed field and -2 for the right-handed field) will have the following neutral electroweak interactions:

$$
\begin{aligned}
-\bar{l}_{L} & {\left[\left(-g \frac{1}{2} \sin \theta_{W}+\frac{g^{\prime}(-1)}{2} \cos \theta_{W}\right) \not A+\left(-g \frac{1}{2} \cos \theta_{W}-\frac{g^{\prime}(-1)}{2} \sin \theta_{W}\right) \not\right] l_{l} } \\
& -\bar{l}_{R} \frac{g^{\prime}(-2)}{2}\left(\cos \theta_{W} \mathcal{A}-\sin \theta_{W} \not\right) l_{R} \\
& =-e A_{\mu} J_{E M}^{\mu}-\frac{e}{2 \sin \theta_{W} \cos \theta_{W}} Z_{\mu} J_{Z}^{\mu}
\end{aligned}
$$

where the electromagnetic current $J_{E M}^{\mu}$ is

$$
J_{E M}^{\mu} \equiv \bar{l} \gamma^{\mu} l
$$

with the electromagnetic charge $e$ defined by

$$
e \equiv g \sin \theta_{W}=g^{\prime} \cos \theta_{W}
$$

and where the neutral weak current $J_{Z}^{\mu}$ is

$$
J_{Z}^{\mu} \equiv \bar{l} \gamma^{\mu}\left(v_{L}-a_{L} \gamma^{5}\right) l
$$

with

$$
\begin{aligned}
v_{L} & \equiv \frac{1}{2}\left(1-4 \sin ^{2} \theta_{W}\right) \\
a_{L} & \equiv \frac{1}{2} .
\end{aligned}
$$

Similarly we can derive the neutral weak current for the neutrinos and quarks (using their respective hypercharges).

We finish these derivations with the charged weak current term. The charged piece of the covariant derivative for the left-handed fields is

$$
i g \frac{\sigma_{1}}{2} W_{\mu}^{1}+i g \frac{\sigma_{2}}{2} W_{\mu}^{2}=\frac{i g}{\sqrt{2}}\left(\begin{array}{cc}
0 & W_{\mu}^{+} \\
W_{\mu}^{-} & 0
\end{array}\right) .
$$

Thus, the full charged weak lepton interaction term within the Lagrangian is

$$
\begin{aligned}
-\frac{g}{\sqrt{2}}\left(\begin{array}{c}
\overline{\nu_{e}} \\
\bar{e}
\end{array}\right)_{L}^{T}\left(\begin{array}{cc}
0 & W^{+} \\
W^{-} & 0
\end{array}\right)\left(\begin{array}{c}
\nu_{e} \\
e
\end{array}\right)_{L} & =-\frac{g}{2 \sqrt{2}}\left(\overline{\nu_{e}} W^{+}\left(1-\gamma^{5}\right) e+\text { h.c. }\right) \\
& =-\frac{g}{2 \sqrt{2}}\left(W_{\mu}^{+} J_{C C}^{\mu}+\text { h.c. }\right)
\end{aligned}
$$

where the charged weak current $J_{C C}^{\mu}$ is

$$
J_{C C}^{\mu} \equiv \overline{\nu_{e}} \gamma^{\mu}\left(1-\gamma^{5}\right) e
$$

In the late 1960s, many authors were looking at electroweak theories similar to those written down above but only containing the charged bosons $W^{ \pm}$and no neutral $Z$. At 
the time, these theories were developed to account for weak-like interactions (i.e. those who violate parity through a vector minus axial-vector coupling to fermions). But, the theories suffered from non-physical behaviors which could be ascribed to nonrenormalizability. In a series of seminal papers [2, 3], Weinberg and others showed how the addition of well-controlled triple-boson interactions could cancel-out the non-desirable behavior in such a away that the theory would become renormalizable, unitary, and gauge invariant.

In particular, Gell-Mann, Goldberger, Kroll, and Low (GGKL) analyzed neutrino to $W$ annihilation [4]. A leading-order contribution to the process comes from the weak interaction (see diagram (a) of Figure 1.2). A theory which includes only weaklyinteracting charged-bosons was found to violate unitarity through the neutrino to $W$ annihilation. In particular, one looks at the partial-wave amplitudes for the process and finds that some of them grow with the center-of-mass energy (a direct violation of unitary) such that, for sufficiently large energy, there becomes a larger than onehundred percent probability of the annihilation occurring. Of course, such a theory does not correspond with reality within and beyond the energy scale at which unitarity is violated.

Weinberg demonstrated how the addition of a weakly interacting neutral-boson would cancel out the poorly behaving pieces of the partial-wave amplitudes and, in doing so, restore the unitarity of the electroweak sector. Below, as we show how this is achieved, we will further obtain a useful illustration for future work in Chapter 2. The process is $\bar{\nu}_{e} \nu_{e} \rightarrow W^{+} W^{-}$and is shown in Figure 1.2.
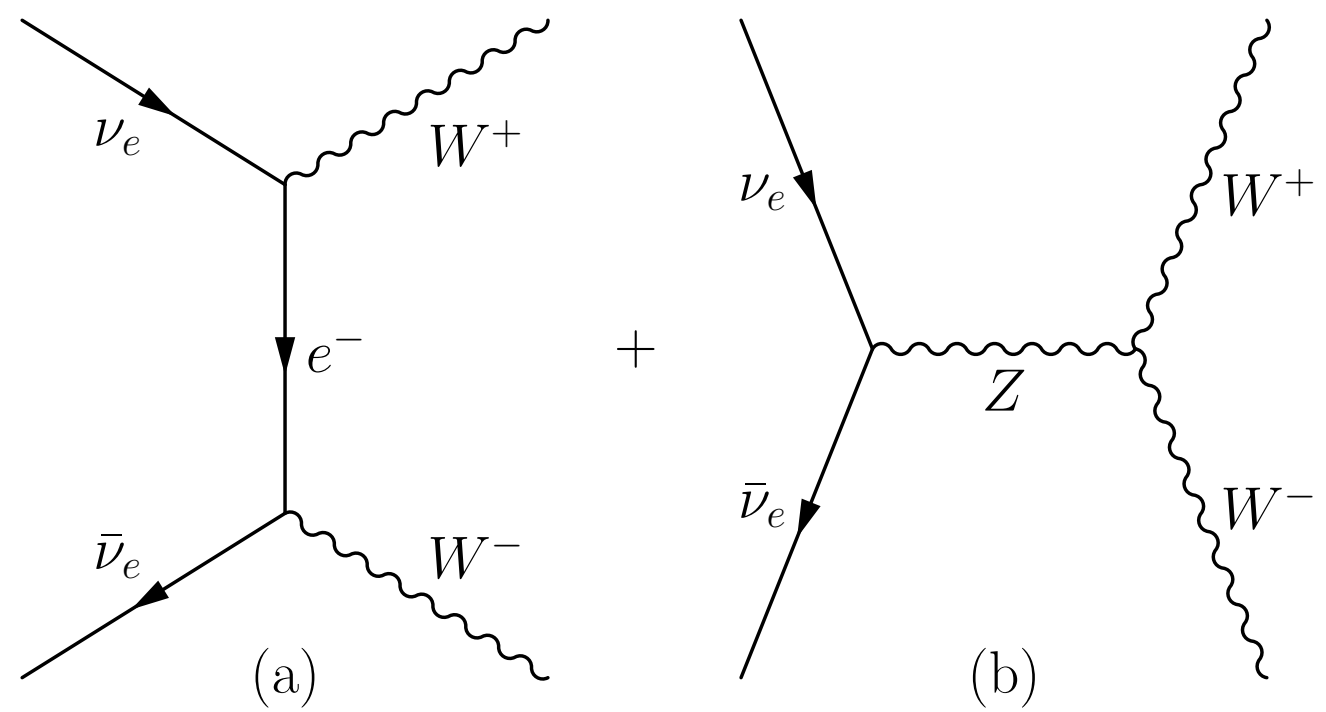

Figure 1.2: The Gell-Mann Goldberger Kroll Low (GGKL) process $\nu \bar{\nu} \rightarrow W^{+} W^{-}$

In the center-of-mass $(\mathrm{COM})$ frame with $W^{+}$energy $E$ and momentum $p$, the 
amplitude squared for (a) solely is

$$
\left|\mathcal{M}_{\mathrm{GGKL},(\mathrm{a})}\right|^{2}=128 G_{F}^{2} p^{2} E^{2} \sin ^{2} \theta\left[\frac{1-(E / p) \cos \theta-m_{W}^{2} /\left(2 E^{2}\right)}{1-(p / E) \cos \theta-m_{W}^{2} /\left(2 E^{2}\right)}\right]^{2},
$$

where

$$
G_{F} \equiv \frac{\sqrt{2} g^{2}}{8 m_{W}^{2}}
$$

is the Fermi coupling constant and $\theta$ is the polar scattering angle of the $W^{+}$relative to the neutrino 3 -momentum (N.B. the process is symmetric in azimuthal angle $\phi$ and we have neglected the electron's mass). At high energy $E$, the (a) differential cross section

$$
\frac{d \sigma}{d \Omega}=\frac{p}{64 \pi^{2} E^{3}}|\mathcal{M}|^{2}
$$

goes like $p^{3} / E \sim E^{2}$. The total cross section, and correspondingly the probability of the (a)-only GGKL process to occur, would then grow like the energy squared. Thus, this (a)-only process violates unitarity.

Put another way, the differential cross section can always be written as a summation of partial squared waves which are intrinsically bounded by their trigonometric phase. Thus, an unbounded process which grows ad infinitum with energy cannot correspond with reality, and violates unitarity. In order to restore unitarity, the full GGKL process must be finite at high energy.

Fortunately, the inclusion of diagram (b), with the neutral $Z$ mediator, saves this dilemma by cancelling out the linear behavior in $E$ within the scattering amplitude, for high energy, such that the full amplitude squared for $(a)+(b)$ is

$$
\begin{aligned}
\mathcal{M}_{\mathrm{GGKL}}^{2} & =128 G_{F}^{2} p^{2} E^{2} \sin ^{2} \theta\left[-\frac{1-(E / p) \cos \theta-m_{W}^{2} /\left(2 E^{2}\right)}{1-(p / E) \cos \theta-m_{W}^{2} /\left(2 E^{2}\right)}+\frac{1+m_{W}^{2} /\left(2 E^{2}\right)}{1-m_{Z}^{2} /\left(4 E^{2}\right)}\right]^{2} \\
& \rightarrow 72 m_{W}^{4} G_{F}^{2} \sin ^{2} \theta, E^{2} \gg m_{W}^{2},
\end{aligned}
$$

which is finite as $E \rightarrow \infty$.

This is one example of how a fully renormalizable Yang-Mills theory requires specific triple-boson interactions to rein in any unitarity violations. In particular, we have seen that not only is a Yang-Mills coupling between the gauge bosons and a non-zero VEV Higgs field necessary to generate mass, but we also see that this coupling simultaneously dictates the exact and particular masses and coupling strength necessary to cancel out high-energy behavior in processes like (1.58). 
This relation between Yang-Mills interaction terms and unitarity was analyzed by Llewelyn-Smith [5]. In general, any phenomenological model that includes triple-bosoninteraction terms, and which hopes to eventually be embedded into a fully renormalizable theory, must satisfy certain criteria. That is, one cannot simply throw in an additional gauge boson interaction without immediately running into unitary problems as with (1.55). As we will see in chapter 2 , the inclusion of a hypothetical neutral muonspecific interaction in order to solve the proton radius problem will also necessitate the inclusion of a hypothetical charged W-like boson.

\subsection{The Strong Sector}

The internal makeup of atomic nuclei strongly depends on a non-electroweak-based interaction we cleverly name the strong force. At short distances (roughly less than 1 femtometer), such as those inside of the proton, the strong force is two orders of magnitude larger in effect than QED. Without the binding force of the strong interaction, baryons, such as protons and neutrons, and mesons would not exist.

Analogous to electroweak, we build the theory of the strong force by adding to the Lagrangian a scalar formed from a set of eight massless spin-1 boson fields $G_{\mu \nu}^{a}$, which we call gluons, corresponding with the $N^{2}-1$ generators of our soon-to-be-imposed $\mathrm{SU}(3)$ symmetry where $N=3$. The scalar we consider is $G_{\mu \nu}^{a} G_{a}^{\mu \nu}$ whose respective eight field-strength tensors, $G_{\mu \nu}^{a}$, are built from the gluon fields which we define to transform under the non-abelian special-unitary gauge group $\mathrm{SU}(3)$. That is,

$$
G_{\mu \nu}^{a}=\partial_{\mu} G_{\nu}^{a}-\partial_{\nu} G_{\mu}^{a}+g_{s} f^{a b c} G_{\mu}^{b} G_{\nu}^{c}
$$

As with the SU(2) fields, the gluons interact amongst each other with a magnitude of the field-strength $g_{s}$ and with a "direction" dependent on the SU(3) structure constants $f^{a b c}$ defined by

$$
\left[T^{a}, T^{b}\right]=i f^{a b c} T^{c}
$$

where

$$
T^{a}=\frac{\lambda^{a}}{2}
$$

and were $\lambda^{a}$ denote the well-known three-by-three Gell-Mann matrices, the $\mathrm{SU}(3)$ equivalent of $\sigma_{i}$, the Pauli matrices of $\mathrm{SU}(2)$. 
The gluons move through free-space and interact with each other through the cubic and quadratic terms in the kinetic piece of the Lagrangian,

$$
\mathcal{L}_{\text {Kinet. }}=-\frac{1}{4} G_{\mu \nu}^{a} G_{a}^{\mu \nu}
$$

where, as before, repeated indices are implicitly summed over. And where, as before, to maintain gauge invariance, the fields must transform as

$$
T^{a} G_{\mu}^{a} \rightarrow U\left[T^{a} G_{\mu}^{a}-\frac{i}{g_{s}} U^{\dagger} \partial_{\mu} U\right] U^{\dagger}
$$

where the rotations in $\mathrm{SU}(3)$ are given by

$$
U(x) \equiv \exp \left[-i T_{a} \alpha^{a}(x)\right]
$$

with eight arbitrary functions $\alpha^{a}(x)$ of space-time position $x$.

Though this self-interacting $\mathrm{SU}(3)$ theory is plenty interesting on its own, we now add interactions with the constituent particles of strongly-bound matter, the quarks, in order to reflect nature. Like leptons, the quarks are spin $1 / 2$ fermions.

Unlike leptons, each quark comes in three varieties of "color". We choose a simple basis for the quarks in this three-dimensional color space, such that each quark can be "color-charged" with three colors (e.g. red, green, and blue; corresponding to $\left(\begin{array}{lll}1 & 0 & 0\end{array}\right)$, $\left(\begin{array}{lll}0 & 1 & 0\end{array}\right)$, and $\left.\left(\begin{array}{lll}0 & 0 & 1\end{array}\right)\right)$.

To add interactions, we modify the covariant derivative within the interaction-piece of the Lagrangian

$$
\mathcal{L}_{\text {Int. }}=\bar{\psi}(i \not D-m) \psi
$$

in the standard way

$$
D_{\mu} \rightarrow \partial_{\mu}-i g_{s} T_{a} G_{\mu}^{a}
$$

where $m$ is the mass for each quark (and can be generated via the Higgs mechanism as described in the previous section 1.1).

Note that, as with $\mathrm{SU}(2)$, the field strength $g_{s}$ within the field-strength tensor (1.59) removes any freedom we have to define the magnitude of color-charge for each quark.

Of course, as the covariant derivative varies under transformations of the gluon field, the fermions must also transform in a similarly covariant manner as

$$
\psi(x) \rightarrow U(x) \psi(x)
$$

where the unitary transformation $U$ is defined in (1.64). 
We have a complete theory of the strong force, which we now call Quantum Chromodynamics (QCD), whose Lagragian is

$$
\mathcal{L}_{\mathrm{QCD}}=i \bar{\psi}(\not D-m) \psi-\frac{1}{4} G_{\mu \nu}^{a} G_{a}^{\mu \nu}
$$

Like leptons, it is understood we implicitly sum over all generations of quarks.

We move on to look at some unique properties of QCD which will entirely motivate our work in Chapter 3. Consider a freely propagating quark shown in Figure 1.3.

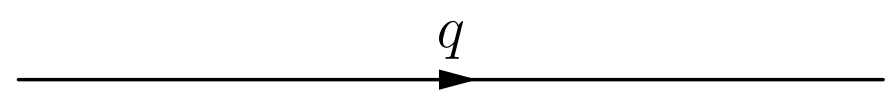

Figure 1.3: Freely propogating quark

As with the leptons in QED, the quark interacts with itself by emitting virtual gluons (along with virtual photons, of course) as shown in Figure 1.4. These virtual gluons, emit virtual quarks again, which reabsorb back into virtual gluons, and so on, eventually being reabsorbed back into the physical quark. The entire effect of these virtual gluon-quark loops, just as in QED, will be to screen the overall color-charge of the quark as anti-color virtual quarks will align themselves closer to the quark, and like-color virtual quarks further away, such that a probe at a long-distance will see an overall-color slightly less than the bare real quark's.

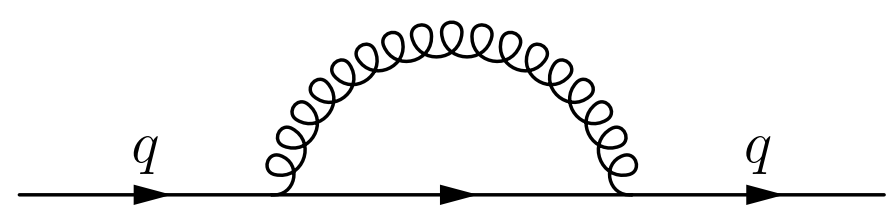

Figure 1.4: Gluon-loop corrections to the quark propagator 
If these gluon-quark loops were the only corrections to the quark propagator, we would then expect the QCD analog of the fine structure constant

$$
\alpha_{s} \equiv \frac{g_{s}}{4 \pi}
$$

to become small and finite over long-distance interactions (i.e. low-energy interactions) just as in QED and infinite at short-distances (high-energy). That is, at one-loop,

$$
\alpha_{s}\left(Q^{2}\right) \sim \alpha_{s}(0)\left\{1+\frac{\alpha_{s}(0)}{3 \pi} f\left(Q^{2} / m_{q}^{2}\right)\right\}
$$

where $Q^{2}$ is the squared four-momentum-transfer. Assuming only gluon-quark loop corrections, the function $f$ increases as $Q^{2}$ increases, thus codifying the our naive expectation of the high-energy running of the QCD coupling constant as in QED.

This would be very nice indeed as we would be able to calculate the so-called "bare" coupling constant at low-energy (a region experimentalists find far more cost-effective to probe than high-energy), obtain a value for $\alpha_{s}$, and use standard renormalization techniques at high-energy (i.e. taking the difference between two theoretical values to cancel any encountered infinities).

But, unlike QED, the virtual gluon emitted by the freely propagating quark also interacts with itself through the triple-boson and quadruple-boson vertices encountered within the QCD field strength tensor (1.62) (see Figure 1.5).

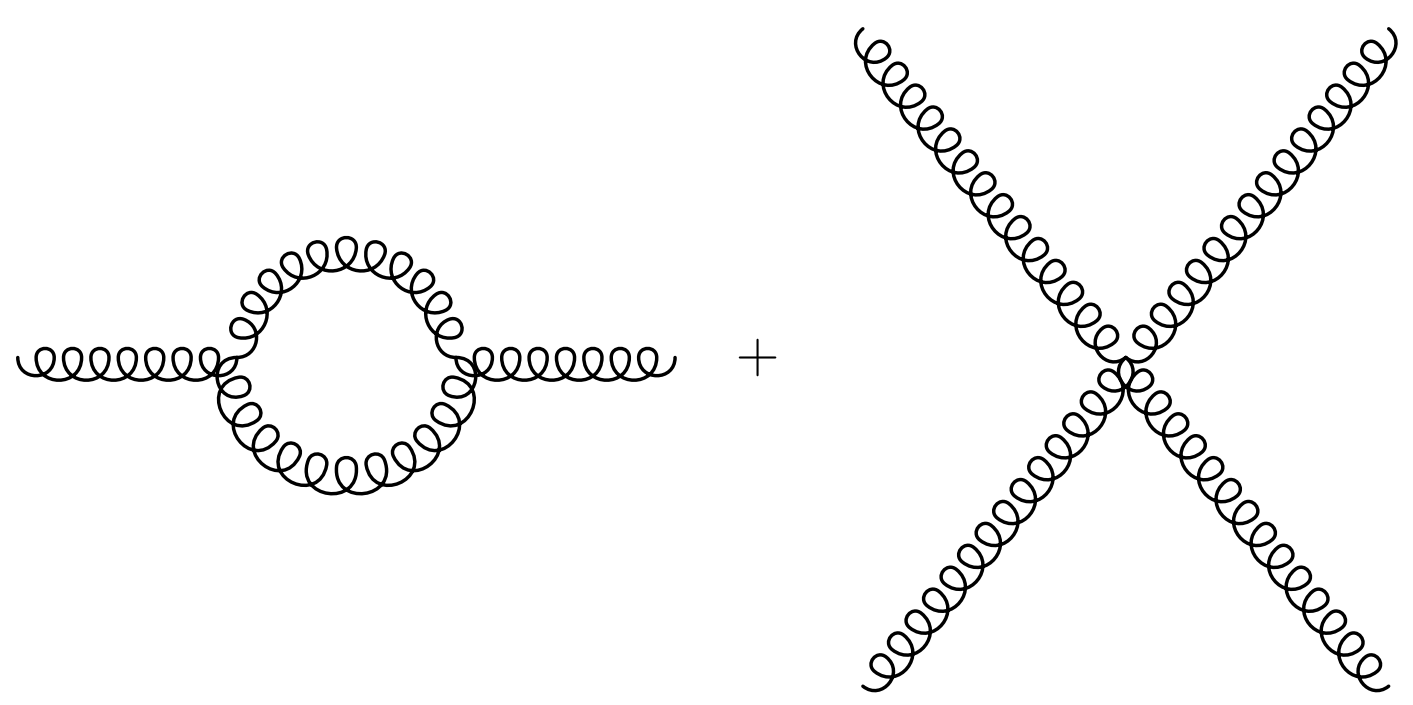

Figure 1.5: Gluonic Self Interactions

Furthermore, the loop corrections to the quark propagator from gluonic self interactions have the opposite effect in screening the effective color-charge of the physical quark. And, this anti-screening effect is stronger than the QED-like screening effect. That is, for long-distance interactions, the effective coupling constant becomes large. 
And for short-distance (high-energy) interactions, the effective coupling constant becomes small, eventually zero as $Q^{2} \rightarrow 0$.

This immediately brings to light a fundamental issue with our naive expectation for the behavior of $\alpha_{s}$ written in equation (1.70). That is, we cannot use a first-order (or any order) calculation to derive the behavior of $\alpha_{s}$ since $\alpha_{s}$ grows large for small $Q^{2}$ and thus $\alpha_{s}$ is not a small quantity around which we can apply perturbation theory to understand its behavior.

Instead, we write the full non-perturbative expectation for the behavior of $\alpha_{s}$ based on the perturbative findings that $\alpha_{s}$ grows large for small $Q^{2}$ and becomes asymptotically small for large $Q^{2}$. Furthermore, since $\alpha_{s}(0)$ is no longer a well-defined quantity, we need to choose another scale, denoted $\Lambda^{2}$, around which we can safely define the behavior of $\alpha_{s}$. That is,

$$
\alpha_{s}\left(Q^{2}\right)=\frac{\alpha_{s}\left(\Lambda^{2}\right)}{1+\alpha_{s}\left(\Lambda^{2}\right) f\left(Q^{2} / \Lambda^{2}\right)},
$$

where we've now codified all loop corrections for small $\alpha_{s}\left(\Lambda^{2}\right)$ through the Taylor series $1 /\left(1+\alpha_{s}\left(\Lambda^{2}\right) f\left(Q^{2} / \Lambda^{2}\right)\right)$.

N.B. This final manipulation requires carefully calculating one-loop gluon corrections to the quark propagator and from this inferring that $f(x) \sim \ln (x)$.

To gain an understanding of the scale $\Lambda$, let us consider the limit where $\Lambda$ is chosen such that

$$
\alpha_{s}\left(Q^{2}\right)=1 / f\left(Q^{2} / \Lambda^{2}\right)
$$

This is the scale where $\alpha_{s}\left(\Lambda^{2}\right)=\infty$. We will denote this scale $\Lambda_{\mathrm{QCD}}$, the QCD "scale parameter", where

$$
\Lambda_{\mathrm{QCD}} \sim 200 \mathrm{MeV} .
$$

For $Q^{2}$ above $\Lambda_{\mathrm{QCD}}$, perturbation theory can be safely applied as $\alpha_{s}$ is less than one. Below $\Lambda_{\mathrm{QCD}}$, QCD is non-perturbative.

We are left with two consequences. One, for small-distances (less than about 1 femtometer), the strong interaction becomes perturbative. Furthermore, for high-energy interactions such as those in deeply-inelastic scattering, the effective-coupling constant becomes small such that we can effectively ignore QCD effects at leading order. In this regime, we say the quarks are "asymptotically free".

And two, over large-distances (more than 1 femtometer), the effective coupling constant becomes larger than one and we can no longer understand QCD through 
perturbative techniques. In this regime, the scale of the interactions dominates over the bare Lagrangian coupling constant $g_{s}$. And the tending-towards-infinite attraction between the quarks results in "confinement." It is for this reason quarks are always observed in bound states of baryons and mesons.

N.B. one may ask why, in this regime, two quarks always appear to be attracted towards one another rather than repulsed. Unfortunately, because the regime is nonperturbative, we do not yet have a clear intuition for why this occurs - though perturbative calculations up to the QCD scale demonstrate the attraction between a quark and an antiquark with one-gluon exchange and how three quarks will align themselves in such a way that two-quarks become color "positive" and the remaining single quark, color "negative", in order to generate an attraction if a single quark is pulled away.

The non-perturbative nature of QCD at low-energies is a difficult hurdle for theorists to overcome. The low-energy regime is the preferred regime of experimentalists. In order to gain an understanding of nature at this scale, experimentalists must be able to subtract the "known" behavior of nature in order to reveal the unknown. If theory cannot tell us anything about the "known" behavior, experimentalists cannot easily discover new physics.

Fortunately, in the recent decades, numerous non-perturbative techniques have been developed to calculate observables from QCD at the nuclear scale. In Chapter 3, we discuss the subtleties surrounding one such technique, a computation-based approximation known as Lattice Quantum Chromodynamics (LQCD).

The "lattice" is a numerical, four dimensional, finite box within which we place the theory of QCD. Space-time is defined in Euclidean coordinates, in order to convert the integrations over the time-evolution operator into classically statistical quantities, and the continuum discretized onto a grid. There are subtle consequences of this procedure encountered when matching the LQCD observable back to the physical observables found in nature. Chapter 3 contains our analysis of one such encountered subtlety in the context of loop-corrections to the quark momentum distribution within nuclear matter. 


\section{Chapter 2}

\section{The Proton Radius Puzzle}

HE implicit summation over leptonic generations in (1.15) implies the identical nature of each lepton in regards to its interactions. This phenomena we call lepton universality and it has been a long standing benchmark of the standard model. So far as we know, only the leptonic mass generation via the leptonHiggs Yang-Mills interaction (1.41) breaks this pattern. However, recent efforts using muons to measure both the proton's electric charge radius $[6,7,8]$ (denoted $R_{E}$ ) and the muon's anomalous magnetic moment (denoted $\left.a_{\mu}\right)[9,10,11,12]$ have prompted an examination of the consistency of lepton universality.

Historically experiments have measured the proton radius via electron scattering and ordinary hydrogen atomic level spectroscopy (for example, a non-point-like proton will affect the size of the Lamb shift, in addition to other observables, of ordinary hydrogen due to the decrease of the hydrogen bound state energy within the $2 S$ orbital from a non-zero charge radius of the proton causing the electron to experience less attraction while "inside" the proton) [13]. All pre-2016 experiments are consistent with one another within their respective statistical and systematic uncertainties. One of the more recent and precise measures of the proton radius comes from electron-proton scattering experiments at Mainz, in which the experimentalists themselves infer the proton radius to be $[8,7]$

$$
R_{E}^{\mathrm{Mainz} .}=0.879(8) \mathrm{fm}
$$

The 2014 CODATA recommended values, along with the 2018 Particle Data Group values, summarize the electric proton radius from electron-proton interactions to be [14, $15]$ 


$$
R_{E}^{\mathrm{PDG}, 2018} \equiv R_{E}^{e}=0.8751(61) \mathrm{fm}
$$

Throughout this work, we will take (2.2) to be the referred to and accepted value of the proton's electric-charge radius as measured by electron-proton interactions.

Recent improvements in methods and technology have allowed experimentalists to measure the same proton radius by observing the Lamb shift within muonic hydrogen rather than ordinary hydrogen. Muons, being about two-hundred times heavier than electrons, are bound closer to the proton and thus are affected by its size more dramatically; allowing experimentalists to achieve new levels of precision. This high-precision experiment resulted in an approximately $4 \%$ smaller proton radius of $[16,6,17]$

$$
R_{E}^{\mu}=0.84087(39) \mathrm{fm} .
$$

As with (2.2), we will take (2.3) as the accepted value for the proton's electric-charge radius as measured by muon-proton interactions.

From (2.2) and (2.3), and with a confidence of $5.6 \sigma$, the proton radius as measured by muon-proton interactions is

$$
\delta R_{E}=0.0342(61) \mathrm{fm}
$$

smaller than the proton radius as measured by electron-proton interactions. The origin of the discrepancy remains an open question.

This is not the only example of a discrepancy involving muon interactions. There is a $3.5 \sigma$ difference between the measured anomalous magnetic moment of the muon [18, 19, 20, 21, 13, 15, 22],

$$
a_{\mu}^{\text {Exp. }}=11659209.1(5.4)_{\text {Stat. }}(3.3)_{\text {Syst. }} \cdot 10^{-10}
$$

by experiment and that predicted by the standard model $[9,12,15]$,

$$
a_{\mu}^{\mathrm{SM}}=116591823(43) \cdot 10^{-11}
$$

In conjunction with the proton electric-charge radius discrepancy (2.4), there exists a hint of possible leptonic universality violation. Jumping at any opportunity to explore new physics, theorists have proposed several beyond-the-standard-model (BSM) models (see [23] for a review) to resolve both problems simultaneously.

One straightforward solution to the proton radius puzzle is the introduction of a new force whose coupling differs between the generations of leptons. In particular, such 
a force must be weakly coupled to the electron compared to the muon. Though the idea is straightforward, known experiments place a plethora of constraints [24, 25, 26] on even the smallest whiff of lepton-universality violation.

A simple scheme involving BSM force carriers that only couple to muons on the leptonic side and only couple to first generation quarks on the hadronic side (additional couplings are taken to be zero or small), has been proposed and studied by Batell et al. [27, 28]. The particular details of these models create corrections to the muonium hyperfine splitting $[29,26]$ and the ${ }^{133} \mathrm{Cs}$ weak charge, as we shall discuss later. These BSM corrections provide experimental constraints on such models. However, the constraints can be avoided by modifying the particular pattern of parity violation in conjunction with a smaller electron coupling.

A new BSM force carrier that couples to muons will alter the muon's one-loop vertex correction, relevant to the $g-2$ discrepancy (to be further discussed in detail within Section 2.1). The degree to which the one-loop vertex is corrected is suppressed by the mass of the new BSM mediator. For instance, a light BSM mediator, of about 1 $\mathrm{MeV}$, can account for the proton radius discprenacy and simultaneously the muon's anomalous magnetic moment discrepancy [28]. A heavy mediator will necessitate the need for an additional BSM force carrier to solve the muon $g-2$ puzzle, and some fine tuning to assure the required cancellation to keep the size of the correction at the proper order of magnitude [27, 30]. Either way, employing at least one new BSM force carrier to solve the proton radius puzzle has the benefit of providing a possible solution the muon's anomalous magnetic moment discrepancy simultaneously.

Eventually, any new BSM model will have to be combined with the Standard Model in order to create a more complete theory. This necessitates that the scattering or decay amplitudes involving the new BSM mediator do not grow with energy. That is, the theory must be gauge invariant and renormalizable.

Karshenboim et al brought attention to this requirement by studying radiative corrections to heavy particle decays such as $W \rightarrow \mu \nu$ [26], which provide constraints on parameters of BSM bosons that may create new channels of decay. Such decays are already well predicted by the standard model with an uncertainty of about $2 \%$.

The result of Karshenboim's work showed that models only involving new BSM low mass (one to one-hundred $\mathrm{MeV}$ ) vector mesons that couple only to muons and protons are ruled out by constraints by radiative corrections to the $W$ 's decay width. An analysis of their work, to be discussed shortly, reveals that the $W$ 's decay width constrains such models because such models lead to corrections to the $W$ decay that grow with energy 
(not only does this violate experimental $W$-decay constraints, but also perturbative unitarity, as the energy grows - to be discussed more in the following section). Thus, a complete solution to the proton radius puzzle and the muonic $g-2$ discrepancy requires additional couplings, along with perhaps additional fields, to give a renormalizable theory whose contributions to scattering amplitudes do not grow with energy for light BSM mediators (i.e. the new theory is unitary and potentially renormablizable).

In 2.1 we review the necessary background to understand the proton radius sufficiently before going on to introduce a vector (plus axial-vector) based solution in 2.2. Additionally, we provide analysis of an alternative scalar (plus pseudoscalar) based solution in 2.3. And in 2.4, we conclude our discussions on potential BSM solutions to the proton radius and muon anomalous magnetic moment discrepancies.

\subsection{The details of the puzzle}

Herein, we dissect the context within which our puzzle exists. We start with what is commonly called the proton's electric charge radius, denoted $R_{E}$. Intuitively, we imagine the proton's constituent quarks occupying a probabilistic "cloud" of space determined by their respective wavefunctions. An incoming particle scattering off the proton will differ in behavior whether inside or outside this constituent-quark cloud. Sufficiently outside this region, the proton can be represented as a point-like charge just as in classical electromagnetism. Inside the region, we must consider the specific, and likely complex, distribution of quarks bound together within the proton. We now translate this intuition of proton size into formal scattering theory.

Consider an electron with low energy non-relativistically scattering off a point-like proton (Figure 2.1). The cross section for this process is the classical Rutherford cross section

$$
\left.\frac{d \sigma}{d \Omega}\right|_{\text {Rutherford }}=\left(\frac{\alpha}{4 E \sin ^{2}(\theta / 2)}\right)^{2}
$$

where

$$
\alpha \equiv \frac{e^{2}}{4 \pi}
$$

is the electromagnetic fine-structure constant, $E$ is the incoming electron's energy, and $\theta$ is the relative scattering angle between the incoming and outgoing electron. 


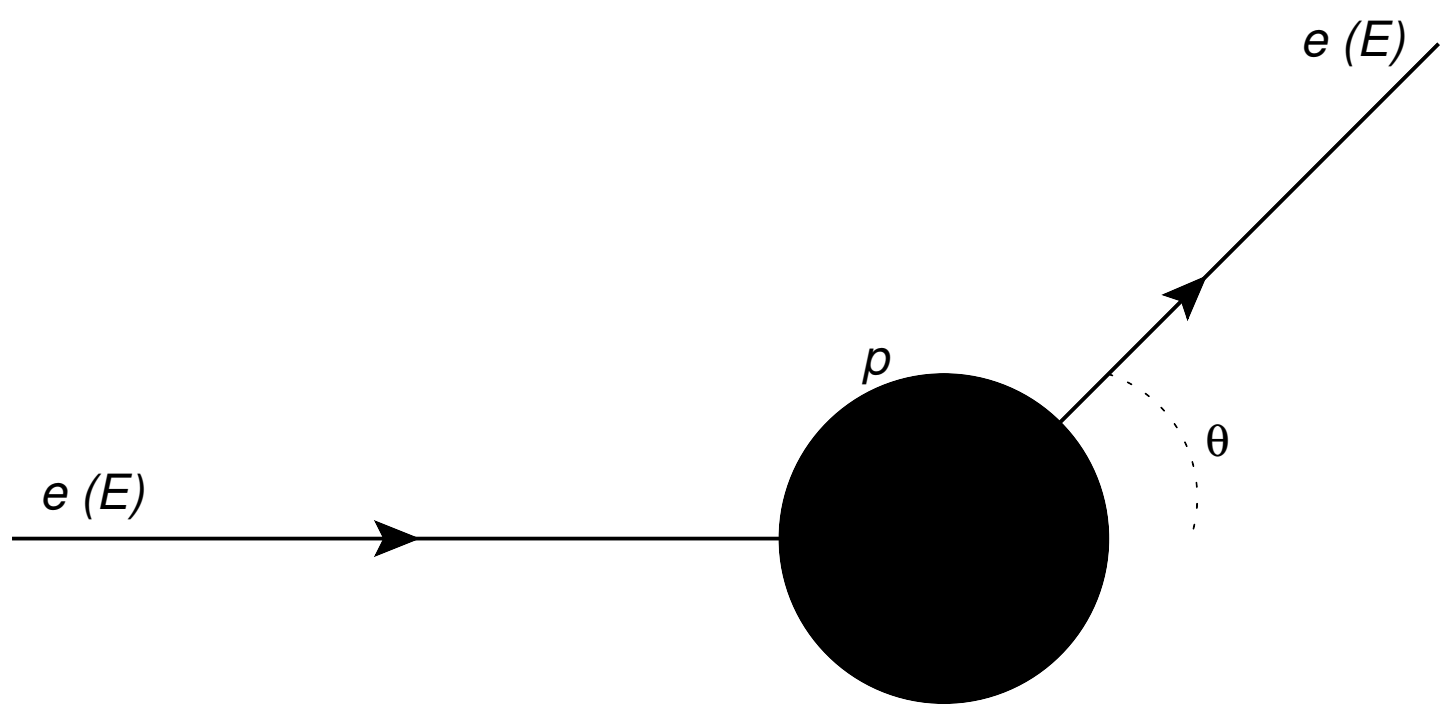

Figure 2.1: Rutherford Scattering: an incoming electron with energy E elastically scatters off a point-like proton target with scattering angle $\theta$.

We expect that as we increase the amount of momentum transfer $Q$, the electron becomes more and more likely to interact within the region encompassed by the constituent quarks. We thus modify our Rutherford differential cross section by a form factor denoted $G\left(Q^{2}\right)$ which has this $Q^{2}$ dependence embedded, that is

$$
\frac{d \sigma}{d \Omega}=\left.G^{2}\left(Q^{2}\right) \frac{d \sigma}{d \Omega}\right|_{\text {Rutherford }} .
$$

In the nonrelativistic limit, the form factor is the Fourier transform of the respective proton's wave function, such that

$$
G\left(Q^{2}\right)=\int d^{3} r e^{i \vec{Q} \cdot \vec{r}}|\psi(r)|^{2},
$$

where $\psi(r)$ describes a spherically-symmetric electromagnetic charge density of the proton as a function of radial distance.

Expanding the form factor for low $Q^{2}$, we write

$$
G\left(Q^{2}\right)=1-\frac{1}{6} r^{2} Q^{2}+\ldots
$$

From this expansion, we thus come across a natural definition for the radius of the proton:

$$
R^{2} \equiv r^{2}=-\left.6 \frac{d G}{d Q^{2}}\right|_{Q^{2}=0}
$$

The energies at which scattering experiments operate require the consideration of the full relativistic process. We thus write down at first order (i.e. one-photon exchange), the relativistic scattering amplitude shown in Figure 2.2 for an incoming electron of with 4-momentum $l$ scattering off a proton with 4-momentum $p$ resulting in an 
outgoing electron with 4-momentum $l^{\prime}$ and outgoing proton with 4-momentum $p^{\prime}$,

$$
\mathcal{M}=\frac{e^{2}}{Q^{2}}\left\langle l^{\prime}\left|j_{\mu}(0)\right| l\right\rangle\left\langle p^{\prime}\left|j^{\mu}(0)\right| p\right\rangle .
$$

Furthermore, the nucleon vertex $\left\langle p^{\prime}\left|j^{\mu}(0)\right| p\right\rangle$ can be written in terms of the Dirac and Pauli form factors ( $F_{1}$ and $F_{2}$ respectively),

$$
\left\langle p^{\prime}\left|j^{\mu}(0)\right| p\right\rangle=\bar{u}\left(p^{\prime}\right)\left[\gamma^{\mu} F_{1}\left(Q^{2}\right)+i \sigma^{\mu \nu} \frac{q_{\nu}}{2 M} F_{2}\left(Q^{2}\right)\right] u(p),
$$

where $\sigma^{\mu \nu}=\frac{i}{2}\left[\gamma^{\mu}, \gamma^{\nu}\right]$. To obtain a physical interpretation for the radius of the proton, we rewrite the Dirac and Pauli form factors in terms of the Sachs form factors denoted $G_{E}$ and $G_{M}$ and respectively defined as

$$
\begin{gathered}
G_{E}=F_{1}-\tau F_{2}, \\
G_{M}=F_{1}+F_{2},
\end{gathered}
$$

which can non-relativisticly be interpreted as the electronic and magnetic charge distributions respectively in the Breit frame (the zero-energy-transfer frame). Here $\tau$ is defined as

$$
\tau \equiv \frac{Q^{2}}{4 M^{2}}
$$

The differential cross section, at leading order, for process shown in Figure 2.2 (also known as the Rosenbluth formula [31]) is

$$
\frac{d \sigma}{d \Omega}=\left.\frac{\left(G_{E}^{2}\left(Q^{2}\right)+G_{M}^{2}\left(Q^{2}\right) \tau / \epsilon\right)}{1+\tau} \frac{d \sigma}{d \Omega}\right|_{\mathrm{Mott}},
$$

where the Mott differential cross section describes the process for a electron scattering relativistically off a point-like proton, and where

$$
\epsilon^{-1} \equiv 1+2(1+\tau) \tan ^{2} \frac{\theta}{2} .
$$

From the first-order differential cross section, we identify two natural definitions for a proton "size" depending on whether we are interested in its electric or magnetic charge distribution.

Herein we are concerned with the electric radius and so we define

$$
R_{E}^{2} \equiv r^{2}=-\left.6 \frac{d G_{E}}{d Q^{2}}\right|_{Q^{2}=0} .
$$

With sufficient understanding of the proton's electric charge radius, we move on to deriving the necessary tools which allow us to measure the radius; and, in doing so, 


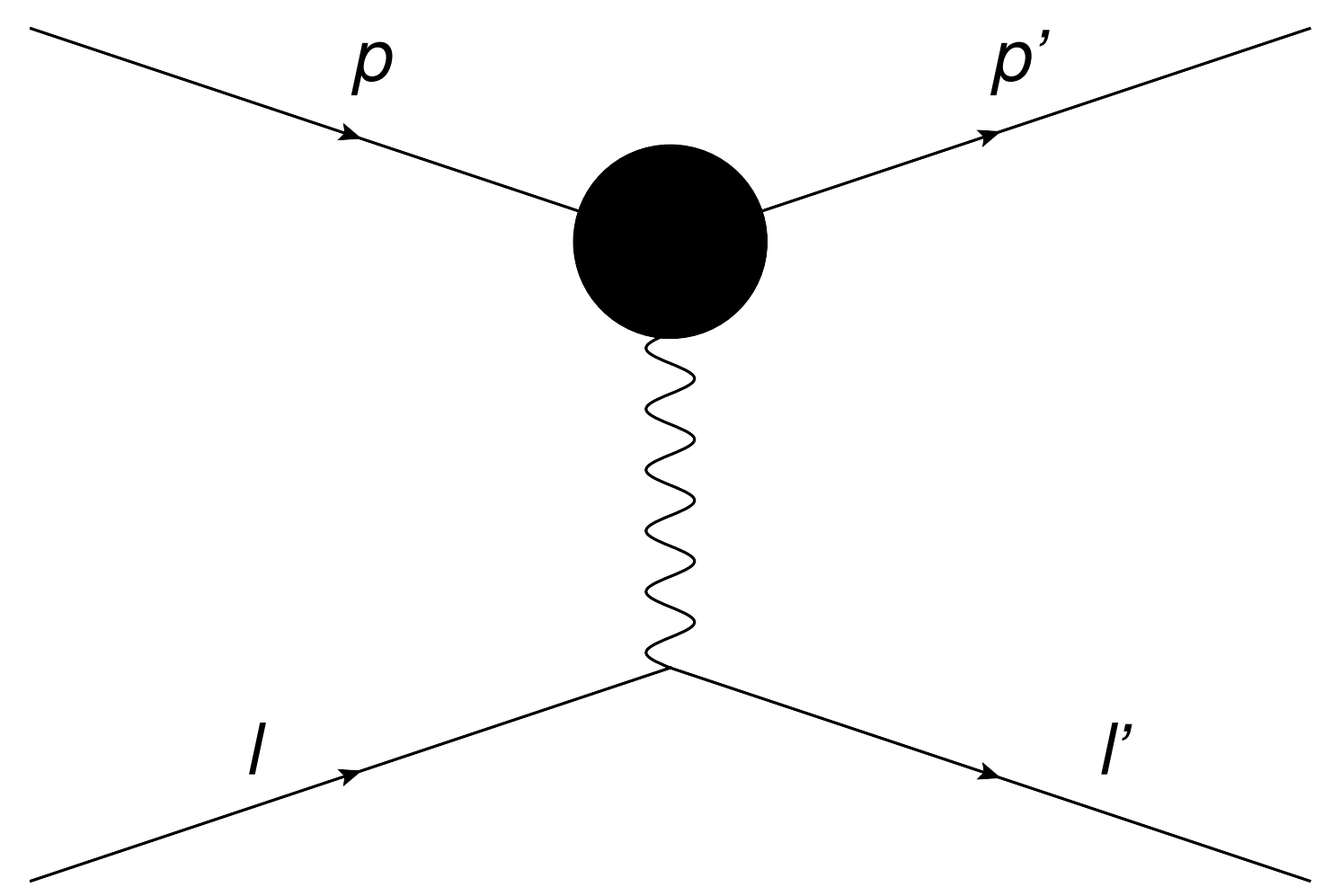

Figure 2.2: 1st order contribution to releativistic electron-proton scattering, the result of which is the Rosenbluth formula. An electron with incoming momentum $l$, and outgoing momentum $l^{\prime}$, scatters off a proton with incoming momentum $p$, and outgoing momentum $p^{\prime}$.

will demonstrate how BSM interactions may mask themselves as discrepancies in the proton radius.

One of the methods used to measure the proton radius is through the $R_{E}$-dependent energy shift in the $S$ state of the proton which reveals itself, for example, when observing the $2 S-2 P$ Lamb shift. In the $S$ orbital, unlike non- $S$ orbitals, the electron has a high probability of being close to the proton and thus its interactions will be noticeably affected by the proton's size. This affect is even further amplified in muonic hydrogen, since the muon, being orders of magnitude heavier than the electron, orbits the proton orders of magnitude closer; thus leading to some of the incredible precision recent muonic-hydrogen-based experiments have achieved [16].

One can calculate with basic quantum mechanics or standard perturbation theory the energy shift of the $2 S$ state resulting from a non-point-like proton with radius $R_{E}[32]$. The shift positively increases (correlating with our intuition that the hydrogen atom will be less bound as the proton's size increases) the energy $2 S$ state by

$$
\Delta E_{S}=\frac{2 \alpha}{3}\left(\frac{m_{r} \alpha}{2}\right)^{3} R_{E}^{2}
$$


where $m_{r}$ is the reduced mass of the bound system and where $R_{E}$ can be shown to have the fully relativistic definition of $(2.17)$ [33, 34].

In 2010, a high precision muonic hydrogen spectroscopy experiment and a flurry of accompanying analytics $[6,8,16]$ found $\Delta E_{S}$ to be approximately $310 \mu \mathrm{eV}$ smaller than that observed in ordinary hydrogen.

In this work, we will analyze one plausible explanation for this discrepancy by introducing a BSM attractive force between the muon and proton which masques itself as lowering $\Delta E_{S}$ by the necessary $310 \mu \mathrm{eV}$.

For example, if we consider an attractive $\mu$-e Yukawa interaction with coupling strength $C$, then there will be an addition shift in the $2 S$ energy state by $[28,27,30]$

$$
\Delta E_{\mathrm{BSM}}=-\frac{C^{2}}{4 \pi} \frac{m_{\phi}^{2}\left(m_{r} \alpha\right)^{3}}{2\left(m_{\phi}+m_{r} \alpha\right)^{4}},
$$

where $m_{\phi}$ is the mass of our BSM mediator. One needs only then to analyze the $\left(C, m_{\phi}\right)$ parameter space necessary to set

$$
\Delta E_{\mathrm{BSM}}=-310 \mu \mathrm{eV}
$$

and consider the problem solved. Of course, it is not that simple. This explains one phenomenon and we must consider the full range of experiments.

\subsection{A renormalizable vector theory}

We begin by introducing two new mediators that couple to the muon and proton explicitly. The first mediator is a vector boson denoted $\phi_{V}$ with coupling strengths $C_{V}^{\mu}$ for the muon and $C_{V}^{p}$ for the proton. The second mediator is an axial vector boson with the same notation as its vector partner, taking $V \leftrightarrow A$. The notation $\phi$ (or $C$ ) without any subscript is understood to represent the vector or axial vector bosons (or couplings) within this section.

To make the decay $W \rightarrow \mu \nu \phi$ gauge invariant, we introduce a third boson, so-named the "shadow W" and denoted $W_{s}$, which interacts with the $\phi$ and the ordinary $W$. The coupling strengths of this 3-boson interaction must be equal to $C^{\mu}$. Furthermore, for gauge invariance, we must also have $m_{W_{s}}=m_{W}$ (hence the name "shadow W").

The additional interactions involving the fields above we write as an addition to the electroweak of standard model Lagrangian

$$
\mathcal{L} \supset \mathcal{L}_{E W}+\mathcal{L}_{\text {int }}
$$


where

$$
\begin{aligned}
\mathcal{L}_{i n t}= & -\phi_{\lambda}^{V}\left[C_{V}^{\mu} \bar{\psi}_{\mu} \gamma^{\lambda} \psi_{\mu}+C_{V}^{p} \bar{\psi}_{p} \gamma^{\lambda} \psi_{p}\right] \\
& -\phi_{\lambda}^{A}\left[C_{A}^{\mu} \bar{\psi}_{\mu} \gamma^{\lambda} \gamma_{5} \psi_{\mu}+C_{A}^{p} \bar{\psi}_{p} \gamma^{\lambda} \gamma_{5} \psi_{p}\right] \\
& -i C_{V}^{\mu} \epsilon_{i j k} W_{\alpha}^{\prime i} W_{\beta}^{\prime j} \partial^{\alpha} W^{\prime k, \beta}+i\left\{C_{A}^{\mu} \text { terms }\right\} \\
& -\frac{g}{2 \sqrt{2}} \bar{\psi}_{\mu} \gamma^{\lambda}\left(1-\gamma_{5}\right) \psi_{\nu} W_{s, \lambda}^{-}+\text {h.c. },
\end{aligned}
$$

and where $\mathcal{L}_{E W}$ is defined in (1.15).

In this notation, within the $C_{V}^{\mu}$ terms, we denote

$$
\begin{aligned}
W_{\alpha}^{\prime 1} & \leftrightarrow W_{\alpha}^{-}, \\
W_{\alpha}^{\prime 2} & \leftrightarrow W_{s, \alpha}^{+}, \\
W_{\alpha}^{\prime 3} & \leftrightarrow \phi_{\alpha}^{V},
\end{aligned}
$$

and with $V \rightarrow A$ for the $C_{A}^{\mu}$ terms. As standard, $\epsilon_{i j k}$ is the antisymmetric Levi-Civita symbol. Note that in this vector plus axial-vector theory, we only require one shadow $W$ for gauge invariance. Furthermore the difference in sign between the the $C_{V}^{\mu}$ and $C_{A}^{\mu}$ Yang-Mills terms is additionally required for gauge invariance. As the last necessity for gauge invariance, we include an interaction term between the $W_{s}$ and charge changing muon current just as is required for the $Z$ and the neutrino current (see (1.58)). We neglect additional interactions between the shadow $W$ and other lepton generations as they are not required in the present context.

In order to produce an attractive force between the muon and proton that would have been mistakenly identified as a decrease in the proton's radius, we fix the relative sign of $C_{V}^{\mu}$ and $C_{V}^{p}$ to be opposite one another such that the muon and proton's interaction with $\phi_{V}$ produces an attraction. A Yukawa attractive force between the muon and proton with a potential of the form

$$
\mathrm{V}_{\mathrm{Y}}(r)=-\frac{C_{V}^{\mu} C_{V}^{P}}{4 \pi} \frac{e^{-m_{\phi} r}}{r}
$$

will contribute to the Lamb shift by

$$
\Delta E(2 S-2 P)=\int_{0}^{\infty} \operatorname{dr} r^{2} \mathrm{~V}_{\mathrm{Y}}(r)\left[R_{20}^{2}(r)-R_{21}^{2}(r)\right]
$$

where $m_{\phi}$ is the mass of the BSM mediator and

$$
\begin{aligned}
& R_{20}(r)=\left(\frac{1}{8}\right)^{1 / 2}\left(\alpha m_{r}\right)^{3 / 2}\left(2-r \alpha m_{r}\right) e^{-r \alpha m_{r} / 2} \\
& R_{21}(r)=\left(\frac{1}{24}\right)^{1 / 2}\left(\alpha m_{r}\right)^{3 / 2}\left(r \alpha m_{r}\right) e^{-r \alpha m_{r} / 2}
\end{aligned}
$$


are the radial pieces of the muonic hydrogen's wave function at the $2 S$ and $2 P$ energy levels respectively with

$$
m_{r} \equiv \frac{m_{\mu} m_{p}}{m_{\mu}+m_{p}}
$$

defined as the muonic hydrogen's reduced mass. The size of this $2 S-2 P$ Lamb shift is $[28,27,30]$

$$
\Delta E(2 S-2 P)=-\frac{\left|C_{V}^{\mu} C_{V}^{p}\right|}{4 \pi} \frac{m_{\phi}^{2}\left(m_{r} \alpha\right)^{3}}{2\left(m_{\phi}+m_{r} \alpha\right)^{4}},
$$

where $\alpha$ is the fine-structure constant (see equation (2.8)).

Note that there is an additional shift in the $2 S$ bound state from the axial coupling $C_{A}$. However, cancellations severely suppress the magnitude of the axial coupling contribution compared to the vector coupling $C_{V}$, even when the coupling coefficients are within similar orders of magnitude.

Not including any BSM forces, we can sum all the QED bound state and finite-size contributions to the Lamb shift, resulting in [17]

$$
E(2 S-2 P)=209.9779(49)-5.2252 R_{E}^{2}+0.0347 R_{E}^{3} \mathrm{meV},
$$

with the radius measured in femtometers.

For example, the original muonic-hydrogen experiment published in Nature by Pohl, Antogini, et al. measured the $2 S$ to $2 P$ transition within muonic hydrogen to be [6],

$$
E_{\text {Exp. }}(2 S-2 P)=206.2949(32) \mathrm{meV} \text {, }
$$

and extracted the proton electric-charge radius as

$$
R_{E}=0.84184(67) \mathrm{fm}
$$

The proton radius as measured by muonic hydrogen is about $0.035 \mathrm{fm}$ smaller than the proton radius as measured by electronic hydrogen. We wish to find the $\Delta E(2 S-2 P)$ that alleviates this discrepancy by letting

$$
\begin{aligned}
E(2 S-2 P) & \rightarrow 209.9779(49)-5.2252\left(R_{E}^{\mu}\right)^{2}+0.0347\left(R_{E}^{\mu}\right)^{3}+\Delta E(2 S-2 P) \\
& =209.9779(49)-5.2252\left(R_{E}^{e}\right)^{2}+0.0347\left(R_{E}^{e}\right)^{3} \mathrm{meV} .
\end{aligned}
$$

From the experimental electric-charge radius values, $R_{E}^{e}$ and $R_{E}^{\mu}$, as measured by electron and muon interactions respectively (see (2.3) and (2.2)), we find

$$
\Delta E(2 S-2 P)=-0.308(14) \mathrm{meV} .
$$


Thus, in order to align the electronic and muonic results for the proton radius, we must decrease the muonic-hydrogen Lamb Shift by about $310 \mu \mathrm{eV}[6,8]$. From (2.29), we plot the parameter space of $\left(C_{V}, m_{\phi}\right)$ necessary to account for the $310 \mu \mathrm{eV}$ reduction assuming

$$
\left|C_{V}^{\mu}\right|=\left|C_{V}^{p}\right|=C_{V}
$$

for simplicity (and similarly for $C_{A}$ ). This parameter space, within two standard deviations, is shown as the green band outlined by solid lines in Figure 2.3 (the green band outlined by dashed lines, and the shaded red and orange regions, will be explained shortly). 


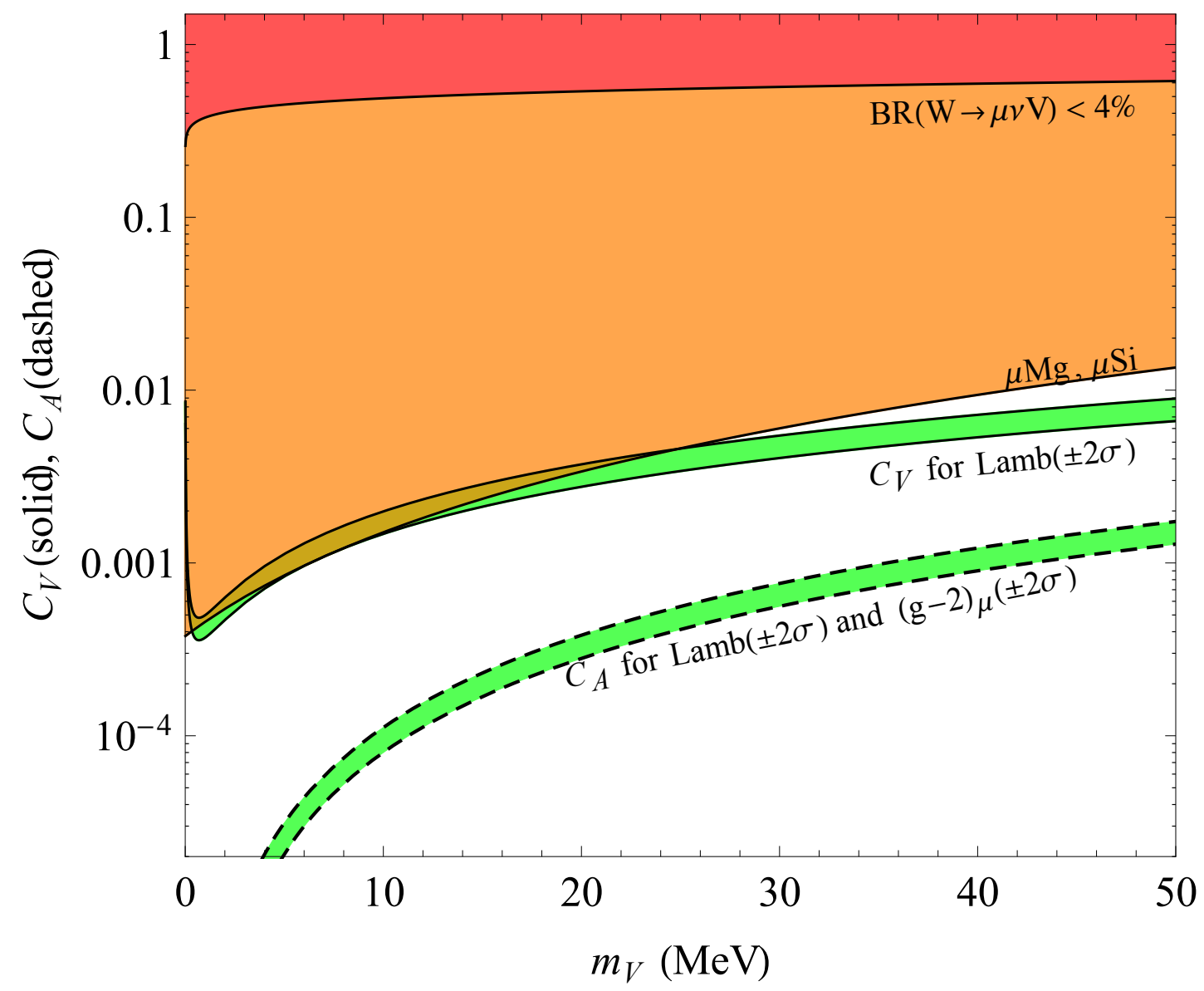

Figure 2.3: The $\left(C_{V}, C_{A}, m_{\phi}\right)$ parameter space necessary to solve the proton radius puzzle and muonic $g-2$ discrepancy while satisfying the considered experimental constraints. The regions bordered be solid black lines refer to constraints on $C_{V}^{\mu}$. The band bordered by dashed lines refer to constraints on $C_{A}^{\mu}$. The green band, outlined by solid black lines, is the region of $\left(C_{V}^{\mu}, m_{\phi}\right)$ required to solve the proton radius problem $( \pm 2 \sigma)$. The shaded red region, bordered below by the black line, is the excluded region of $\left(C_{V}^{\mu}, m_{\phi}\right)$ due to the constraint that the branching ratio for $W$ goes to $\mu \nu \phi_{V}+\mu \nu \phi_{A}$ must be less than $4 \%$ (under the assumption that $C_{A}^{\mu}$ is set to solve the muonic $g-2$ problem). The shaded orange region is the restricted region on $\left(C_{V}^{\mu}, m_{\phi}\right)$ due to energy splittings in muonic $\mathrm{Mg}$ and $\mathrm{Si}$ at $2 \sigma$. The green band, outlined by dashed lines, is the desired region of $\left(C_{A}^{\mu}, m_{\phi}\right)$ which solves solve the muonic $g-2$ problem $( \pm 2 \sigma)$ (under the assumption that $C_{V}^{\mu}$ is set to solve the proton radius problem $( \pm 2 \sigma)$ ).

Furthermore, the introduction of the BSM vector and axial-vector interactions with the muon will shift the muon anomalous magnetic moment

$$
a_{\mu}=\frac{g_{\mu}-2}{2} .
$$

where $g_{\mu}$ is the standard gyromagnetic ratio for muon. At zero'th order (the Dirac equation for the muon), we expect $g_{\mu}=2$. From this expectation, we define $a_{\mu}$ above to capture the small shifts away from $g_{\mu}=2$ due to quantum loop corrections.

Conveniently, we can use these contributions to tune away the known discrepancy from experiment and theory. To understand how this can be accomplished, let us first 
compare $a_{\mu}$ from experiment $[18,19,20,21,13,15,22]$,

$$
a_{\mu}^{\text {Exp. }}=11659209.1(5.4)_{\text {Stat. }}(3.3)_{\text {Syst. }} \cdot 10^{-10},
$$

and that predicted by the theory (the standard model) $[9,12,15]$,

$$
a_{\mu}^{\mathrm{SM}}=116591823(43) \cdot 10^{-11},
$$

with the result from theory being a summation of the QED, weak, and hadronic lowestorder contributions shown respectively in Fig. 2.4 (see [15] for a review).
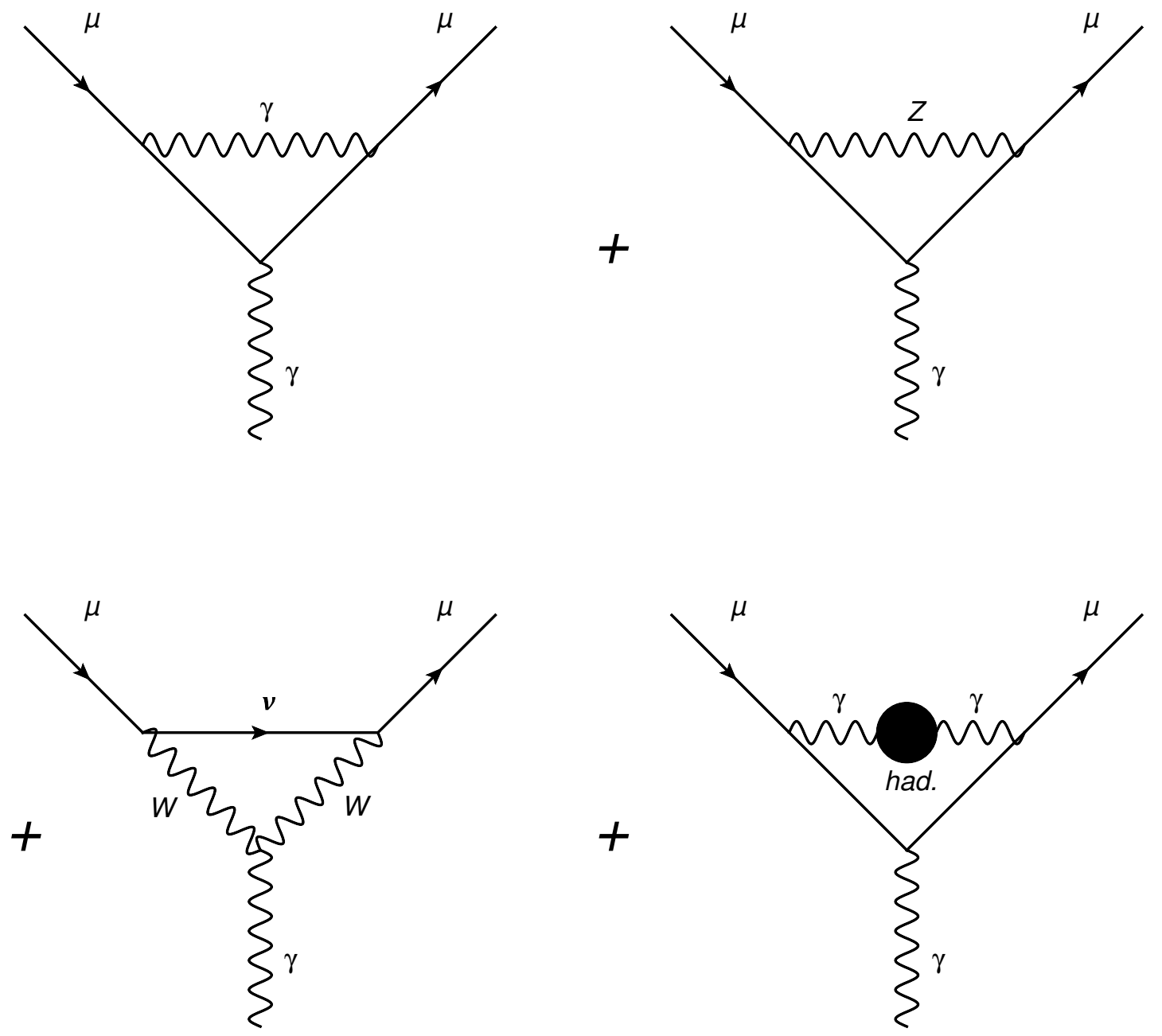

Figure 2.4: Lowest-order contributions to the muon's anamlous magnetic moment from QED, weak, and hadronic interactions respectively.

The muon anomalous magnetic moment discrepancy is then

$$
\begin{aligned}
\delta a_{\mu} & =a_{\mu}^{\mathrm{Exp} .}-a_{\mu}^{\mathrm{SM}} \\
& =268(76) \times 10^{-11} .
\end{aligned}
$$

The introduction of muon-specific vector and axial-vector couplings contributes to the muon's anomalous magnetic moment, shown in Figure 2.5. 


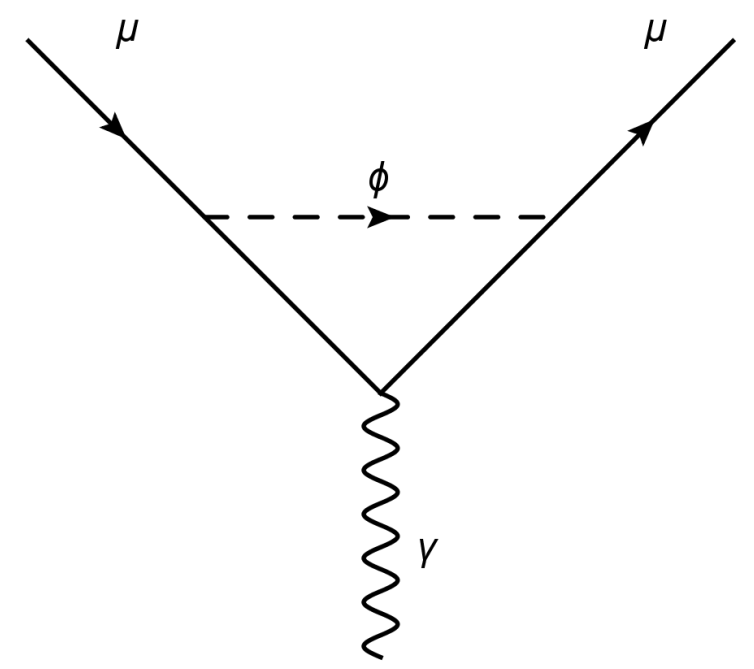

Figure 2.5: BSM contributions to the muon's anomalous magnetic moment. Here $\phi$ repeseents the addition of both vector and axial-vector diagrams.

The size of the contribution is $[35,36]$

$$
\begin{aligned}
\delta a_{\mu}^{\phi} & =\frac{m_{\mu}^{2}}{4 \pi^{2}} \int_{0}^{1} \mathrm{~d} z \frac{C_{V}^{2} z^{2}(1-z)-C_{A}^{2}\left[z(1-z)(4-z)+2 m_{\mu}^{2} z^{3} / m_{\phi}^{2}\right]}{z^{2} m_{\mu}^{2}+(1-z) m_{\phi}^{2}} \\
& =\frac{1}{4 \pi^{2}}\left[C_{V}^{2} H_{V}\left(m_{\phi}^{2} / m_{\mu}^{2}\right)-C_{A}^{2} H_{A}\left(m_{\phi}^{2} / m_{\mu}^{2}\right)\right]
\end{aligned}
$$

where we have assumed equal masses for the vector and axial vector mediators, that is

$$
m_{\phi} \equiv m_{V}=m_{A}
$$

and where

$$
H_{V}(r) \equiv \frac{1-2 r}{2}+\frac{r(r-2)}{2} \ln r-\frac{\sqrt{r}\left(r^{2}-4 r+2\right)}{\sqrt{r-4}} \ln \left(\frac{\sqrt{r}+\sqrt{r-4}}{2}\right)
$$

and

$$
H_{A}(r) \equiv \frac{1}{r}+\frac{2 r-5}{2}-\frac{r^{2}-4 r+2}{2} \ln r+(r-2) \sqrt{r(r-4)} \ln \left(\frac{\sqrt{r}+\sqrt{r-4}}{2}\right),
$$

with

$$
r \equiv m_{\phi}^{2} / m_{\mu}^{2}
$$

Note that the equations (2.42) and (2.43) above continue smoothly for $0<r<4$.

The vector and axial vector couplings affect the anomalous moment with opposite signs in (2.40) and thus they can be tuned to account for the known discrepancy between theory and experiment of muonic $g-2[30]$. Additionally, note how the change to the muon's anomalous magnetic moment (2.40) is suppressed by $1 / m_{\mu}^{2}$ compared to the 
change the Lamb shift (2.29). This allows for a light BSM mediator to contribute to the Lamb shift while negligibly affecting the anomalous magnetic moment.

With $C_{V}$ set to satisfy and constrained by the proton radius problem (2.29), we can then solve for $C_{A}$ in (2.40) by equating $\delta a_{\mu}^{\phi}$ to (2.61).

This desired region for $C_{A}$ that eliminates from the muon $g-2$ discrepancy is described by the green band outlined by dashed lines in Fig. 2.3.

Fortunately, none of the aforementioned constraints forbid the introduction of BSM mediators necessary to solve the proton radius problem and muon anomalous magnetic moment discrepancy. Unfortunately, in 2014, Karshenboim et al. [26], demonstrated that the high-energy behavior within $W$-decay forbids the existence of these BSM mediators (for a given model containing only BSM bosons such as our $\phi_{V}$ and $\phi_{A}$ ).

Specifically, $W$-decay experiments constrain the branching ratio of $W \rightarrow \mu \nu \phi_{V}$ plus $W \rightarrow \mu \nu \phi_{A}$.

Our most precise $W$-decay width measurements come from the Tevatron [37, 38, 39] where experimentalists found the total $W$-decay width to be

$$
\Gamma_{W}=2.085 \pm 0.042 \mathrm{GeV}
$$

Following Karshenboim, we conservatively allow for the $W$-decay width to shift by up to twice the experimental error from the addition of $W \rightarrow \mu \nu \phi$. That is,

$$
B\left(W \rightarrow \mu \nu \phi_{V}+\mu \nu \phi_{A}\right)<4 \%
$$

Without a shadow $W$ and its corresponding triple-boson-interactions, this limit would disallow the region of the $\left(C_{V}^{\mu}, m_{\phi}\right)$ parameter space required to explain the proton radius puzzle (as shown by Karshenboim).

With the shadow $W$, we now calculate the $W \rightarrow \mu \nu \phi$ branching ratio from the Feynman diagrams shown in Fig. 2.6.

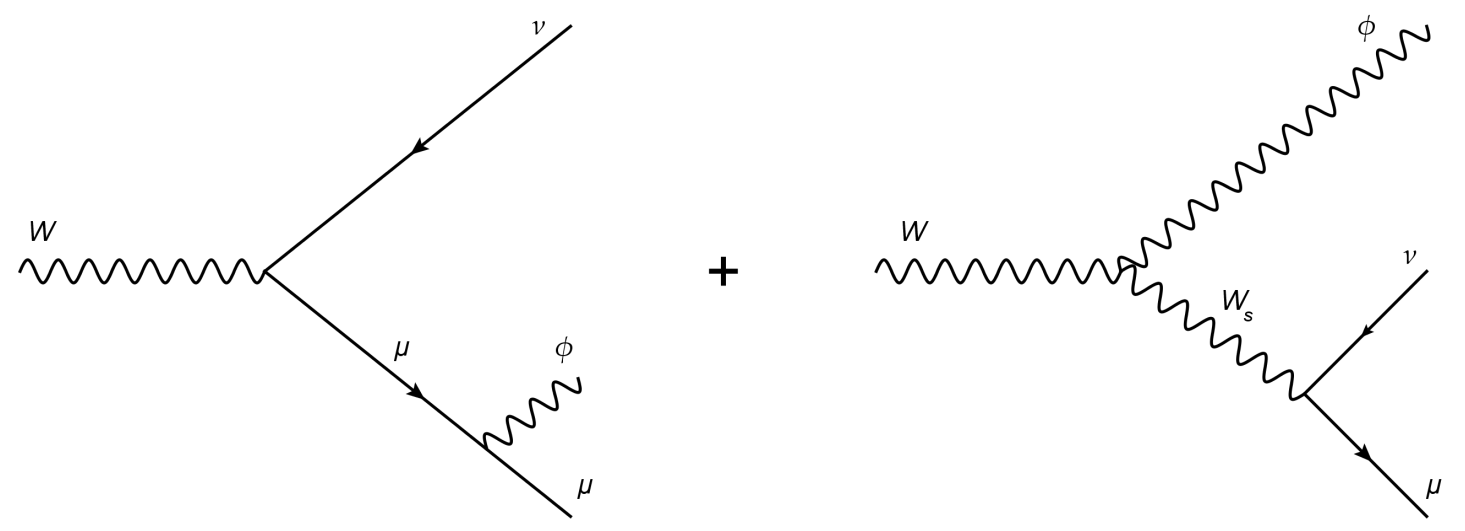

Figure 2.6: BSM contributions to $W$-decay $W \rightarrow \mu \nu \phi$, including the shadow $W$ branch. 
Using the triple-boson interaction Lagrangian given in (2.22) we compute the decay amplitude in 2.6 as

$$
\begin{aligned}
i \mathcal{M}\left(W \rightarrow \mu \nu \phi_{V}\right) & =\frac{i C_{V}^{\mu} g_{W}}{2 \sqrt{2}} \epsilon_{\alpha}(k) \epsilon_{\beta}^{*}\left(p_{3}\right) \bar{u}\left(p_{1}\right)\left\{\frac{\gamma^{\beta}\left(\not p_{1}+\not p_{3}\right)}{\left(p_{1}+p_{3}\right)^{2}} \gamma^{\alpha}\left(1-\gamma^{5}\right)\right. \\
& -\gamma^{\mu}\left(1-\gamma^{5}\right)\left(\frac{g_{\mu \nu}-\frac{\left(p_{1}+p_{2}\right)_{\mu}\left(p_{1}+p_{2}\right)_{\nu}}{m_{W}^{2}}}{\left(p_{1}+p_{2}\right)^{2}-m_{W}^{2}}\right) \\
& \times\left(g^{\alpha \beta}\left(k+p_{3}\right)^{\nu}+g^{\beta \nu}\left(-p_{3}+p_{1}+p_{2}\right)^{\alpha}\right. \\
& \left.\left.+g^{\alpha \nu}\left(-p_{1}-p_{2}-k\right)^{\beta}\right)\right\} \nu\left(p_{2}\right)
\end{aligned}
$$

where $k$ is the $W$ 4-momentum, $p_{1}$ is the muon 4-momentum, $p_{2}$ is the neutrino 4 momentum, and $p_{3}$ is the $\phi_{V}$ 4-momentum. The above decay amplitude is calculated only for an outgoing vector boson $\phi_{V}$. The $W \rightarrow \mu \nu \phi_{A}$ amplitude is equivalent modulo a negative sign, which is squared away within the physically relevant decay width.

For negligible muon and neutrino mass we find

$$
\begin{aligned}
\Gamma_{W}(W \rightarrow \mu \nu \phi) & =\frac{G_{F} m_{W}^{3}\left[\left(C_{V}^{\mu}\right)^{2}+\left(C_{A}^{\mu}\right)^{2}\right]}{96 \sqrt{2} \pi^{3}} \\
& \times\left\{\log ^{2} \frac{m_{W}^{2}}{m_{\phi}^{2}}-5 \log \frac{m_{W}^{2}}{m_{\phi}^{2}}+\frac{37}{3}-\frac{\pi^{2}}{3}\right\},
\end{aligned}
$$

to leading order in $m_{\phi} / m_{W}$. Note additional corrections can occur from non-zero muon mass as multiplicative corrections to the coefficients like $\left(1+\mathcal{O}\left(m_{\mu}^{2} / m_{W}^{2}\right)\right)$.

Without the additional $Z$-like diagram involving the shadow $W$ in Figure 2.6, the decay width would be [26]

$$
\Gamma_{W}(W \rightarrow \mu \nu \phi)=\frac{G_{F}\left[\left(C_{V}^{\mu}\right)^{2}+\left(C_{A}^{\mu}\right)^{2}\right]}{512 \sqrt{2} \pi^{3}} \frac{m_{W}^{5}}{m_{\phi}^{2}}
$$

The decay width in (2.49) contains a $1 / m_{\phi}^{2}$ infrared divergence. Not only does this decay width push the desired parameter space of $C_{V}^{\mu}$ and $m_{\phi}$ far into the excluded region from the experimental $W$-decay constraints (see Figure 6 in [26]), but this decay width also points to violations in unitarity and renormalizibility as the partial wave amplitude which contributes grows with energy.

However, at leading order in $m_{\phi} / m_{W}$, the process which includes the shadow $W$ diagram, as seen in Figure 2.6 and calculated in (2.48), has a logarithimic rather than inverse-polynomial infrared divergence. That is, the $1 / m_{\phi}^{2}$ divergence, which arises from the longitudal polarization of $\phi$, is cancelled by the $W_{s}$ propagator in the 2 nd diagram of Figure 2.6. This cancellation of the infrared divergence simultaneously 
pushes experimentally excluded region away from the allowed parameter space (see Figure 2.3) while eliminating concerns regarding unitarity and renormalizibility.

Furthermore, the axial-vector coupling $C_{A}^{\mu}$ in (2.48) can be obtained in terms of $C_{V}^{\mu}$ by applying the constraint from $(g-2)$ in $(2.40)$. Thus the constraint resulting from the $W$ decay branching ratio can be applied to $C_{V}^{\mu}$ and $m_{\phi}$ exclusively, assuming $C_{A}^{\mu}$ is set to solve the muon anomalous magnetic moment discrepancy. This constraint from $W$ decay which eliminates the allowed parameter space of $\left(C_{V}^{\mu}, m_{\phi}\right)$ is drawn as the shaded red region above the top curve in Fig. 2.3. The values of $C_{V}^{\mu}$ below this red region are allowed within the experimental context of $W$-decay.

An additional constraint on the $\left(C_{V}^{\mu}, m_{\phi}\right)$ parameter space arises as a result of transitions between $3 d$ and $2 p$ orbitals in muonic magnessium ${ }^{24} \mathrm{Mg}$ and muonic silicon ${ }^{28} \mathrm{Si}[40,24,26]$. One precision experiment in the mid 1980s explored beyond-QED and CPT violation within muon-nucleon interactions. No BSM physics was observed and the results remain today as tight constraints on new physics.

To understand where from these constraints come, let us consider a BSM Yukawa potential of the form seen in (2.24). This potential will shift the energy levels between the $3 d$ and $2 p$ states of a given atom by

$$
\frac{\delta \lambda}{\lambda_{\mathrm{QED}}}=-\frac{8 \pi C_{V}^{\mu} C_{V}^{P} A}{5 \alpha Z}[9 f(2)-4 f(3)]
$$

where

$$
f(n)=\left(1+n m_{\phi} / 2 \alpha Z m_{\mu}\right)^{-2 n},
$$

and where $\delta \lambda$ denotes the difference between the $3 d$ to $2 p$ energy shifts as predicted from standard QED and as predicted from QED plus an additional Yukawa interaction. Furthermore, $A$ represents the Atomic number (e.g. 12 for Magnesium and 14 for Silicon). And $n$ denotes the principle quantum number (e.g. $n=3$ for a $3 d$ state and $n=2$ for a $2 p$ state). The 1985 experiment found [40]

$$
\frac{\lambda_{\text {Exp. }}-\lambda_{\mathrm{QED}}}{\lambda_{\mathrm{QED}}}=-0.2 \pm 3.1 \text { parts per million. }
$$

Letting

$$
\left|C_{V}^{\mu}\right|=\left|C_{V}^{p}\right|=C_{V}
$$

one can equate the experiment constraint (2.52) to the BSM contribution (2.50) in order to exclude a certain region of the $\left(C_{V}, m_{\phi}\right)$ parameter space. This restricted region is plotted as the shaded orange area borderd by a solid black line below in Figure 2.3. Allowed values of $C_{V}$ exist below this region. 
One must also consider a common constraint arising from muonium hyperfine splitting, as discussed in [26]. However, this constraint is not applicable within the sampled theory since $\phi$ does not couple to the electron in (2.22).

Similarly, the constraint on $C_{A}^{\mu}$ from BSM contributions to the weak charge in ${ }^{133} \mathrm{Cs}$ as a result of new parity nonconserving interactions, also discussed in [26], is negligible as the parity-violating interactions only contribute at the two-loop and above level. This lessens the restrictions on the allowed parameter space for $\left(C_{A}, m_{\phi}\right)$ compared to theories which contain these parity-violating interactions at tree-level or 1st order.

Focusing on Figure 2.3, one notes that there exists broad regions of allowed parameter space for which we can find values of $C_{V}, C_{A}$, and $m_{\phi}$ that simultaneously solve the proton radius puzzle and the muonic $g-2$ discrepancy without violating the considered experimental constraints.

For completeness, we also consider radiative corrections to $Z \rightarrow \mu^{+} \mu^{-}$decay, specifically $Z \rightarrow \mu^{-} \mu^{+} \phi_{V}$ and $\mu^{-} \mu^{+} \phi_{A}$ decay as represented in Figure 2.7.
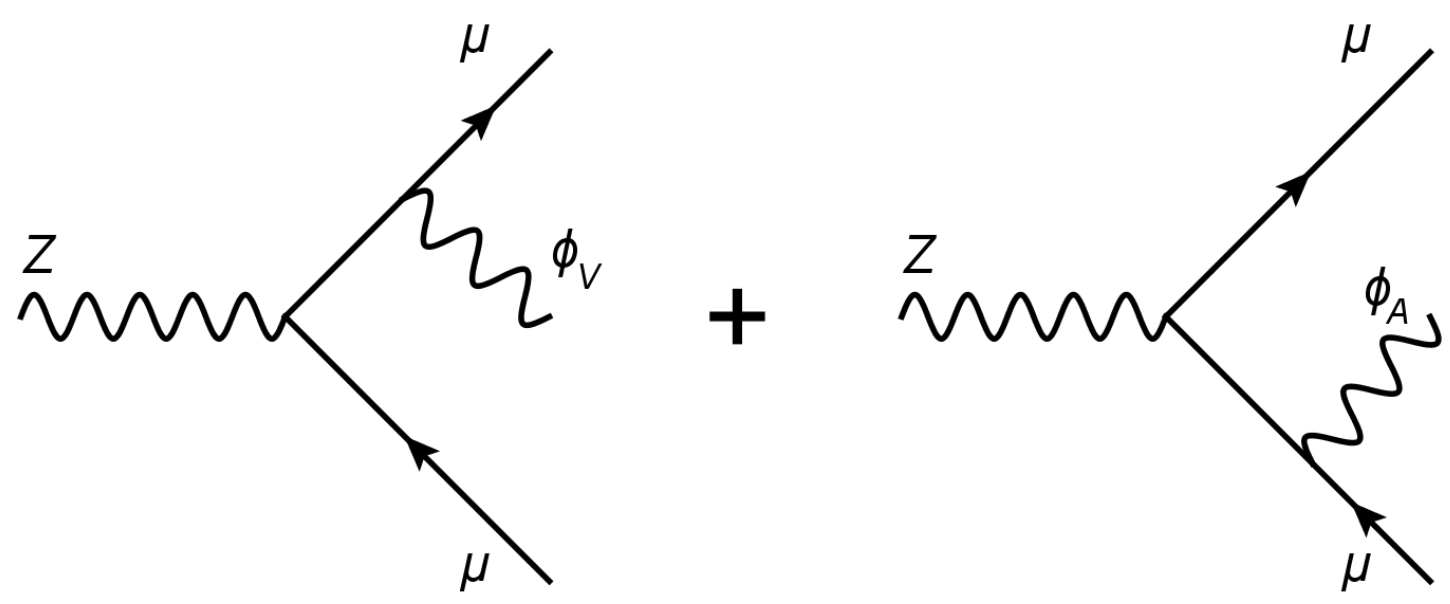

Figure 2.7: BSM contributions to Z-decay: $Z \rightarrow \mu^{-} \mu^{+} \phi$

The decay amplitude for the vector-diagram only is

$$
\begin{aligned}
i \mathcal{M} & =\frac{i}{2} \frac{g_{W}}{\cos \theta_{W}} C_{V}^{\mu} \epsilon_{\alpha}(k) \epsilon_{\beta}^{*}\left(p_{3}\right) \bar{u}\left(p_{1}\right) \\
& \times\left\{\frac{\gamma^{\beta}\left(\not p_{1}+\not p_{3}\right)}{\left(p_{1}+p_{3}\right)^{2}} \gamma^{\alpha}\left(-\frac{1}{2}+2 \sin ^{2} \theta_{W}-\frac{1}{2} \gamma^{5}\right)\right. \\
& \left.-\gamma^{\alpha}\left(-\frac{1}{2}+2 \sin ^{2} \theta_{W}-\frac{1}{2} \gamma^{5}\right) \frac{\left(\not p_{2}+\not p_{3}\right) \gamma^{\beta}}{\left(p_{2}+p_{3}\right)^{2}}\right\} \nu\left(p_{2}\right)
\end{aligned}
$$

where $k$ is the $Z$ 4-momentum, $p_{1}$ is the muon 4 -momentum, $p_{2}$ is the anti-muon 4 momentum, and $p_{3}$ is the $\phi_{V} 4$-momentum. As with the $W$ decay, here we only focus 
on the vector contribution to the $Z$ decay. The axial vector amplitude is equivalent up to an overall minus sign (which is squared away in the observable decay width).

In this case, without any shadow-like contributions (such as those considered in the similar $W$-decay BSM contributions), cancellations between the two diagrams ensure the Ward identity is satisfied. There is no poor behavior at high energies when the $\phi$ is longitudinally polarized. This is seen in the logarithmic dependence of the decay width (2.55) on $1 / m_{\phi}$ (which now resembles that of the $W$ decay (2.48)),

$$
\begin{aligned}
\Gamma_{Z} & =\frac{G_{F} m_{Z}^{3}\left[\left(C_{V}^{\mu}\right)^{2}+\left(C_{A}^{\mu}\right)^{2}\right]\left[\frac{1}{2}-2 \sin ^{2}\left(\theta_{W}\right) \cos \left(2 \theta_{W}\right)\right]}{48 \sqrt{2} \pi^{3}} \\
& \times\left\{\log ^{2} \frac{m_{Z}^{2}}{m_{\phi}^{2}}-4 \log \frac{m_{Z}^{2}}{m_{\phi}^{2}}+5-\frac{\pi^{2}}{3}\right\}
\end{aligned}
$$

Note, we have neglected the muon mass (as in the calculation of the $W \rightarrow \mu \nu \phi$ decay)s, and we have expanded the Z's decay width in (2.55) to leading order in $m_{\phi} / m_{Z}$. These steps are motivated by the arguments provided in the paragraph directly following (2.48).

\subsection{Scalar Theory}

Analogous to the vector theory analyzed in section 2.2, herein we consider the scalar theory equivalent. The scalar theory is conveniently well behaved without the addition of any "shadow" particles, and thus is quite simple in comparison to the previouslydiscussed model. The BSM scalar interaction Lagrangian is

$$
\begin{aligned}
\mathcal{L}_{\text {int }, S} & =\phi_{S}\left[C_{S}^{\mu} \bar{\psi}_{\mu} \psi_{\mu}+C_{S}^{p} \bar{\psi}_{p} \psi_{p}\right] \\
& +\phi_{P}\left[C_{P}^{\mu} \bar{\psi}_{\mu} \gamma^{5} \psi_{\mu}+C_{P}^{p} \bar{\psi}_{p} \gamma^{5} \psi_{p}\right]
\end{aligned}
$$

where $\phi_{S}$ is the scalar field and $\phi_{P}$ is the pseudo-scalar field. The two BSM mediators have, for simplicity, equal mass

$$
m_{\phi_{S}}=m_{\phi_{P}} \equiv m_{\phi}
$$

The constant $C_{S}^{\mu}$ is the coupling strength of the scalar to the muon. The other coupling strengths are given similarly with corresponding superscripts and subscripts denoting the respective standard-model particle BSM particle interaction. Throughout this section, we will often write $\phi$ to represent either $\phi_{S}, \phi_{P}$, or the addition of the two, to be understood in context. 
The BSM scalar plus psuedoscalar theory considered contributes to the Lamb shift of muonic hydrogen by

$$
\Delta E(2 S-2 P)=-\frac{\left|C_{S}^{\mu} C_{S}^{p}\right|}{4 \pi} \frac{m_{\phi}^{2}\left(m_{r} \alpha\right)^{3}}{2\left(m_{\phi}+m_{r} \alpha\right)^{4}},
$$

where $m_{r}$ is the reduced mass of muonic hydrogen (see equation (2.28)) and $\alpha$ is the fine structure constant (see equation (2.8)).

In order to solve the proton radius problem, the change to the Lamb shift must be

$$
\Delta E(2 S-2 P)=-0.308(14) \mathrm{meV} .
$$

See equation (2.29) and explanations surrounding (along with the corresponding references provided) for the derivation of this required $310 \mu \mathrm{eV}$ contribution to the Lamb shift.

From (2.58), we plot the $\left(C_{S}, m_{\phi}\right)$ parameter space necessary to account for the $310 \mu \mathrm{eV}$ reduction of the Lamb shift assuming

$$
\left|C_{S}^{\mu}\right|=\left|C_{S}^{p}\right| \equiv C_{S}
$$

for simplicity (and similarly for $C_{A}$ ). This parameter space, within two standard deviations, is shown as the green band outlined by solid lines in Figure 2.9 (note that the parameter space which solves the proton radius problem for the scalar theory is the same as the parameter space for the vector theory shown in Figure 2.3).

Following a similar analysis to the vector theory, we also consider the shift to the muon's anomalous magnetic moment that the scalar and pseudoscalar interactions will create.

The muon anomalous magnetic moment discrepancy is

$$
\delta a_{\mu}=268(76) \times 10^{-11} .
$$

See equations 2.37 and (2.38) in Section 2.2, along with the arguments supported by references thereabouts, for further explanation.

The introduction of muon-specific scalar and pseudoscalar couplings contributes to the muon's anomalous magnetic moment, shown in Figure 2.8. 


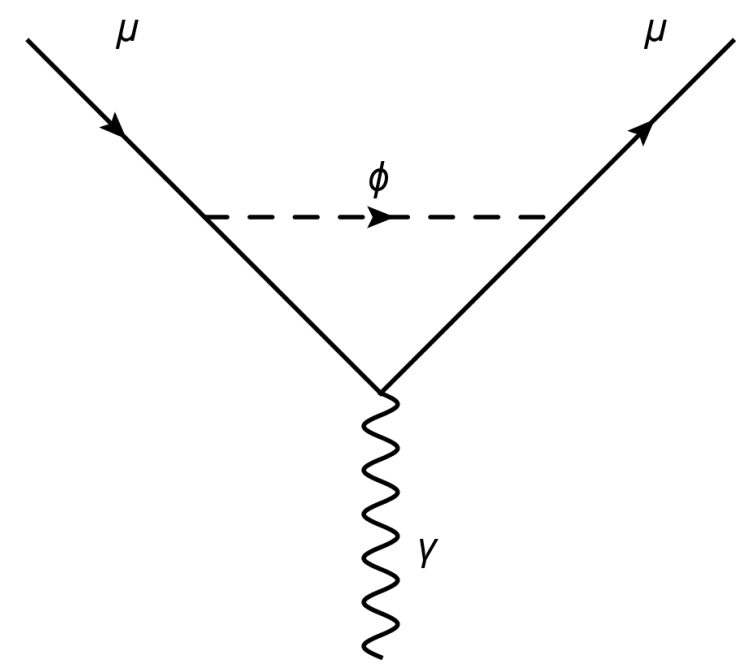

Figure 2.8: BSM contributions to the muon's anomalous magnetic moment. Here $\phi$ repeseents the addition of both vector and axial-vector diagrams.

The BSM scalar plus psuedoscalar contribution from Figure 2.8 is $[35,36]$

$$
\begin{aligned}
\delta a_{\mu}^{\phi} & =\frac{m_{\mu}^{2}}{8 \pi^{2}} \int_{0}^{1} \mathrm{dz} \frac{C_{S}^{2} z^{2}(2-z)-C_{P}^{2} z^{3}}{z^{2} m_{\mu}^{2}+(1-z) m_{\phi}^{2}} \\
& =\frac{1}{8 \pi^{2}}\left[C_{S}^{2} H_{S}\left(m_{\phi}^{2} / m_{\mu}^{2}\right)-C_{P}^{2} H_{P}\left(m_{\phi}^{2} / m_{\mu}^{2}\right)\right],
\end{aligned}
$$

where

$$
H_{S}(r) \equiv \frac{3-2 r}{2}+\frac{r(r-3)}{2} \ln r-(r-1) \sqrt{r(r-4)} \ln \left(\frac{\sqrt{r}+\sqrt{r-4}}{2}\right)
$$

and

$$
H_{P}(r) \equiv-\frac{2 r+1}{2}+\frac{r(r-1)}{2} \ln r-\frac{r^{3 / 2}(r-3)}{\sqrt{r-4}} \ln \left(\frac{\sqrt{r}+\sqrt{r-4}}{2}\right),
$$

with

$$
r \equiv m_{\phi}^{2} / m_{\mu}^{2}
$$

Note that the equations (2.63) and (2.64) above continue smoothly for $0<r<4$.

Assuming $C_{S}$ is set to solve the proton radius problem (by equating (2.58) and $(2.59))$, we then plot the parameter space of $\left(C_{P}, m_{\phi}\right)$ as the dashed green line in Figure 2.9 (for clarity, only the central value is shown). 


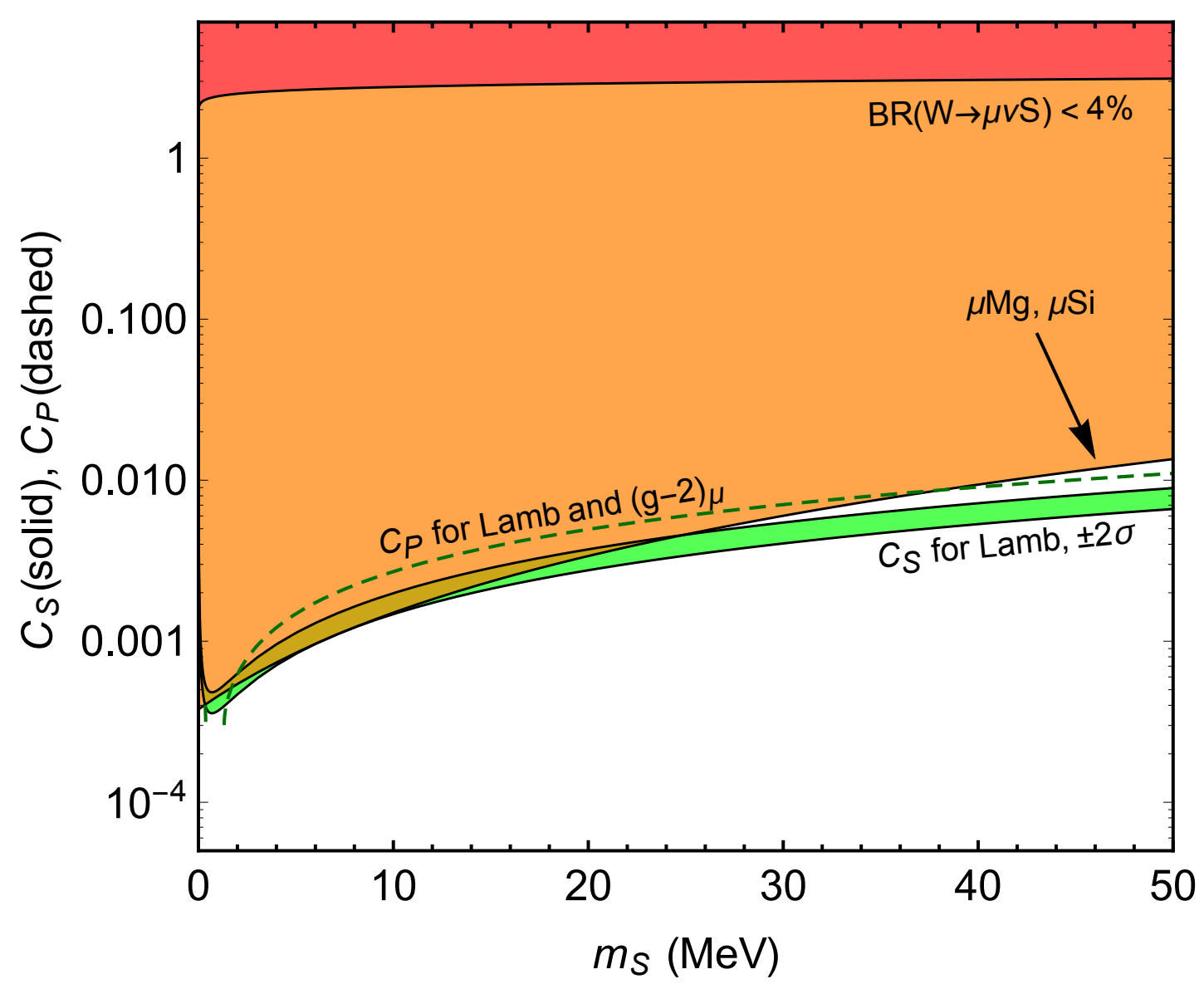

Figure 2.9: The $\left(C_{S}, C_{P}, m_{S}\right)$ parameter space necessary to solve the proton radius puzzle and muonic $g-2$ discrepancy while satisfying the considered experimental constraints. The regions bordered be solid black lines refer to constraints on $C_{S}^{\mu}$. The dashed lines represent constraints on $C_{P}^{\mu}$. The green band, outlined by solid black lines, is the region of $\left(C_{S}^{\mu}, m_{S}\right)$ required to solve the proton radius problem $( \pm 2 \sigma)$. The shaded red region, bordered below by the black line, is the excluded region of $\left(C_{S}^{\mu}, m_{S}\right)$ due to the constraint that the branching ratio for $W$ goes to $\mu \nu \phi_{S}+\mu \nu \phi_{P}$ must be less than $4 \%$ (under the assumption that $C_{P}^{\mu}$ is set to solve the muonic $g-2$ problem). The shaded orange region is the restricted region on $\left(C_{S}^{\mu}, m_{S}\right)$ due to energy splittings in muonic $\mathrm{Mg}$ and $\mathrm{Si}$ at $2 \sigma$. The dashed green line is the desired parameter space of $\left(C_{P}^{\mu}, m_{S}\right)$ which solves solve the muonic $g-2$ problem (under the assumption that $C_{S}^{\mu}$ is set to solve the proton radius problem).

As previously analyzed in Section 2.1, the same $W$-decay constraint on the branching ratio $[26,37,38,39]$

$$
B\left(W \rightarrow \mu \nu \phi_{S}+\mu \nu \phi_{P}\right)<4 \%
$$

similarly restricts the scalar theory.

The decay amplitude for the scalar and psuedoscalar branches is given by the Feynman diagram in Figure 2.10 (note that, compared to the vector theory $W$-decay in Figure 2.6, there is now only one diagram which contributes). 


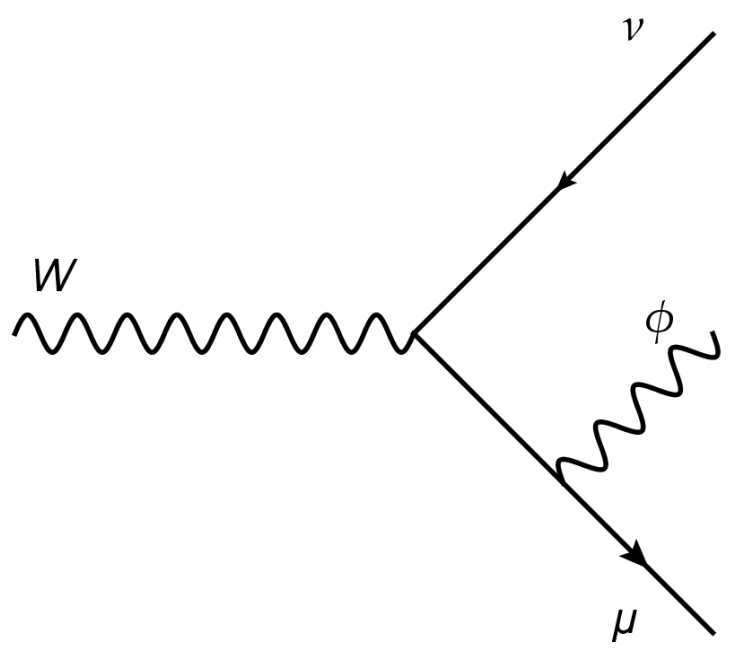

Figure 2.10: Scalar BSM contribution to the $W$-decay $W \rightarrow \mu \nu \phi_{S}+\mu \nu \phi_{P}$. In the figure, $\phi$ represents the addition of the scalar and pseudoscalar contributions.

The decay width is

$$
\Gamma_{W, \text { scalar }}=\frac{G_{F} m_{W}^{3}\left[\left(C_{S}^{\mu}\right)^{2}+\left(C_{P}^{\mu}\right)^{2}\right]}{96 \sqrt{2} \pi^{3}}\left\{\log \left(\frac{m_{W}}{m_{\phi}}\right)-\frac{17}{12}\right\}
$$

where, as with the vector case, we let the muon and neutrino mass go to zero.

The logarithmic dependence in (2.67) pushes the $W$-decay constraint away from the desired parameter space $\left(C_{S}, m_{\phi}\right)$ necessary to solve the proton radius problem. This is seen in the constraint plot Figure 2.9, where the shaded-red region above the black line shows denotes values of $\left(C_{s}, m_{\phi}\right)$ forbidden by this constraint (note that there exists an additional forbidden region, shaded orange, to be described in the paragraphs below). The area in white beneath the black line is the allowed region, and the required parameter space to solve the proton radius problem and the muon anomalous magnetic moment discrepancy (both shown in green) sits mostly inside the allowed region.

the restricted red-shaded area bordered below by the black line sits far above the green-band (representing the preferred parameter space for $C_{S}$ ).

The logarithmic rather than polynomial infrared divergence is similar to that seen in the vector through in (2.48). However, in the vector-theory case, the addition of the so-named "shadow W" was necessary to cancel the undesired infrared divergences previously noted in [26]. For the scalar theory, no such additions are necessary.

The additional constraint resulting from transitions between $3 d$ and $2 p$ orbitals in muonic magnessium ${ }^{24} \mathrm{Mg}$ and muonic silicon ${ }^{28} \mathrm{Si}$ [40, 24, 26] (previously discussed in Section 2.2) also applies to the scalar theory here in exactly the same form. See (2.52) and the surrounding equations and arguments for further insight. 
This constraint excludes a region of the $\left(C_{S}, m_{\phi}\right)$ parameter space and is plotted as the shaded orange area borderd by a solid black line below in Figure 2.9. Allowed values of $C_{S}$ exist below this region.

And, as with the vector-based theory, one should also consider the common constraints arising from muonium hyperfine splitting and from BSM contributions to the weak charge in ${ }^{133} \mathrm{Cs}$ as a result of new parity nonconserving interactions, as discussed in [26]. The former constraint does not apply as the BSM interactions of $\phi$, provided in (2.56), do not include couplings to the electron. And the latter constraint is negligible as the parity-violating interactions only contribute at the two-loop and above level. The lack of these two constraints significantly opens the allowed paramter space of $\left(C_{V / A}, m_{\phi}\right)$, seen in Figure 2.9, compared to theories which contain BSM electron- $\phi$ interactions and/or parity-violating interactions at tree-level or 1st order.

From Figure 2.3, one sees that there exists broad regions of parameter space for which we can find possible values of $C_{S}, C_{P}$, and $m_{\phi}$ that simultaneously solve the proton radius puzzle and the muonic $g-2$ discrepancy without violating the considered experimental constraints.

\subsection{Conclusion}

In the introduction of this chapter, we discussed the proton electric-charge radius discrepancy between recent experiments involving muonic hydrogen spectroscopy and prior experiments involving standard hydrogen spectroscopy and electron-proton scattering. The more-recent muonic-hydrogen-based experiments measured the proton radius to be about $0.034 \mathrm{fm}$ smaller than previously observed, with a confidence level of $5.6 \sigma$ (see equation 2.4 and the references thereabouts).

Furthermore, in the same section, we looked at another closely related puzzle: the $3.5 \sigma$ difference between the muon's anomalous magnetic moment as predicted by the standard model compared to the same as extracted from experiment (see equations 2.5 and 2.6 and the supporting references). The origin of and the solution to the discrepancies became the motivation for the discussed research.

We then provided an overview of possible solutions along with an analysis of some known experimental constraints on the BSM solutions. In particular, we focused on the work of Karshenboim et al. [26]. Therein, it was pointed out that a careful consideration of BSM contributions to the $W$ decay was required, as the decay was likely poorlybehaved in the high-energy limit in addition to being a heavily-restrictive constraint on 
the parameter space of many of the proposed BSM theories.

In order to familiarize the reader with the relevant definitions and derivations, in section 2.1, we analyzed in detail the background theory required to understand the context in which the proton radius puzzle exists.

And, in section 2.2, we brought forth a possible solution to the proton radius puzzle. The solution involved two new BSM mediators, one vector boson and one axial-vector boson, which couple to muons (and not electrons). We also introduced the notion of a "shadow W" boson which behaves analogously to the standard $W$ boson, but only couples only to the muon (in the leptonic sector) and to the other electroweak mediators through a Yang-Mills interaction term. The full interaction lagrangian was given in equation (2.22).

The "shadow W" was a possibly necessary feature for vector-based solutions to the proton radius puzzle, as its interactions reined in the previously-discussed high-energy problems within $W$ decay, which are tied to violations of unitarity and renormalizability. Additionally, the new contribution to $W$ decay, shown in Figure 2.6, pushed the experimentally constraining region away from the desired parameter space to solve the proton radius and anomalous magnetic moment discrepancies. That is, for theories which contain this additional "shadow" Yang-Mills couplings, the non-physical energy dependence of the considered processes is ameliorated, and the constraint arising from BSM contributions to the $W$ decay width no longer causes problems for such models to simultaneously solve the proton radius and muon anomalous magnetic moment puzzles.

The tightest constraint on the vector/axial-vector model considered arises from the measured energy splittings in muonic magnesium and silicon, which are both wellpredicted in the standard model and precisely extracted from experiment. See equation 2.50 and the accompanying arguments and references for further detail. Fortunately, there still remains a large swath of unconstrained $\left(C_{V}, m_{\phi}\right)$ and $\left(C_{A}, m_{\phi}\right)$ parameter space available to solve the two noted discrepancies.

The desired parameter (coded in green) and the experimentally-restricted parameter space (shaded in orange and red) are plotted in Figure 2.3. From this plot, it is fair to conclude that some proposed BSM theories, such as that described by the interaction lagrangian in equation 2.22, which involve new vector and axial-vector bosons, along with at least one additional "shadow" $W$-like boson and an accompanying "shadow" Yang-Mills term, remain a viable phenomenological representation of some underlying complete solution to the proton radius puzzle and the muon anomalous magnetic moment discrepancy. Furthermore, one must consider that the underlying complete so- 
lution which necessarily would be embedded into the standard model must be renormalizable. As observed by Llewellyn Smith [5], the additional Yang-Mills terms provided, involving the "shadow" $W$, is one step in this direction.

In section 2.2, we discuss the scalar/pseudo-scalar analog of the previously considered vector plus axial-vector model. The story is much the same for the scalar theory described by 2.56 , with one notable exception. For the scalar case, the decay width of the $W$ boson does not grow polynomially with energy and thus the scalar theory does not require any additional "shadow" bosons in order to reign in violations of unitarity. The desired parameter, along with the constraints previously discussed, is plotted in Figure 2.9. One notes that just like the vector theory, the scalar theory also remains a viable phenomenological solution to the proton radius puzzle and the muon anomalous magnetic moment discrepancy.

Embedding the vector or scalar phenomenological models into the standard model necessitates new interactions which may lead to additional constraints. For instance, one must include the process through which the new BSM bosons acquire their mass. And one may consider the contribution to the rate of muon-pair production within proton-collider experiments. Furthermore, there will be BSM-loop corrections to the self energy of, for example the $W$. These corrections with loops of BSM particles are correlated with contributions to the Peskin-Takeuchi parameters [41, 42, 43]. The Peskin-Takeuchi parameters are only defined under particular conditions (e.g. for BSM theories involving new heavy particles). But, one can find analogues parameters for theories involving new light particles, such as those so-far considered. And, contributions to these parameters may be calculated and compared against experimental constraints.

Additionally, the new muon-specific interactions will create further radiative corrections beyond those already-discussed in $W$ decay. For instance, one may consider corrections to the decay width of the muon, the pion, and so on. Such decays occur at a significantly lighter mass scale than $W$ decay and thus are less likely to be constrained by known experiment. Nevertheless, one interested in furthering the research discussed may consider such processes.

These additions noted in the two paragraphs above are mentioned here only briefly and are beyond the scope of this thesis. Within the context analyzed, the successes noted by the large region of allowed parameter space in Figure 2.3 and Figure 2.9 point to the remaining viability of these theories as solutions to the proton radius and muon $g-2$ puzzles. 


\section{Chapter 3}

\section{Subtleties within the Euclidean Quark Quasidistribution}

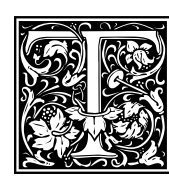

HE Standard Model [44] has had immense success at making accurate predictions of experiment. From the high-precision tests of QED (such as recent lion [45]) to the discovery of the Higgs boson [46, 47, 48, 49], there has been hallmark after hallmark of remarkable achievements. Despite these successes, a notable gap of knowledge remains within our understanding of nuclear and hadronic physics from first principles. In the low energy regime of the strong force (about $200 \mathrm{MeV}$ and below), QCD becomes non-perturbative as the strong coupling constant $\alpha_{s}$ grows larger than one (see Section 3 of Chapter 1 for an introductory review). Our standard set of perturbative-based tools, such as those available in the perturbative high-energy regime [50], are then no longer applicable in making analytic predictions from theory.

Numerous new techniques (e.g. effective field theories such as heavy quark effective theory [51, 52], non-relativistic QCD [53], and soft-collinear effective theory [54]) have been developed to counteract this dilemma. These techniques rely on convoluting shortdistance (high-energy) perturbative effects, calculated by theory, with long-distance (low-energy) non-perturbative effects as measured by precision experiment. Because of the intertwining with experimental output, global QCD analysis is not a first-principles method of predicting nature. We then turn to a modern a priori technique which does not intrinsically rely on experiment.

This non-perturbative technique, known as Lattice Quantum Chromodynamics (LQCD) [55] saw initial success in understanding low-energy quantities such as hadronic mass $[56,57]$ and form factors at near-zero energy transfer $Q^{2}$ [58]. LQCD is fundamentally a numerical algorithm designed to emulate QCD. This is achieved by Wick rotating space-time into Euclidean space such that the field-path integrals become purely statistical quan- 
tities. That is, one can then treat the action in Feynman's path integral formalism [59] as a statistical system.

Furthermore, since LQCD requires numerical computation, space-time must also be discretized and finite in volume. The discretization of space-time provides an intrinsic ultraviolet (short-distance) cutoff which regulates the theory. These features, the Wick rotation and the discretization of space-time onto a finite volume hypercube, place some limitations on the power of LQCD. For example, dynamic systems that explicitly involve interactions over time cannot be readily calculated. Fortunately, much of the QCD we see in nature exists as bound quark states and thus LQCD is sufficient to calculate certain low-energy and time-independent features observed in experiment.

Because LQCD emulates, rather than predicts, the QCD we see in nature, adjustments must be made to the LQCD prediction if we are to compare to experiment. An obvious correction is the extrapolation of discretized space-time onto the continuum, a necessary step in order to remove cut-off / regularization dependence and finite-volume effects. A more subtle correction is the elimination of possible artifacts that may be created from the initial Wick rotation of the action into Euclidean space.

Herein, we explore how to match a particular LQCD observable to its experimental equivalent. Specifically, we demonstrate how to match a lattice calculation of the Euclidean quark quasidistribution function (defined in 3.2) to the physical Minkowskian quark distribution function (defined in 3.2). Along the way, we will discover that the lattice-necessary Wick rotation into Euclidean space causes artifacts in the nonperturbative regime which cannot be corrected for with perturbation theory. Towards the end of this chapter, we will show how these results have motivated recent developments [60] which demonstrate how the statistical nature of the lattice eliminates these perhaps troublesome artifacts.

In 3.1, we begin by reviewing a common probe of QCD physics: deep inelastic scattering (DIS). From the DIS amplitude, we define the parton distribution function and analyze its large-momentum limit, the quasidistribution. In 3.2, we introduce lattice perturbation theory as a tool to analyze the lattice analogues of the parton distribution functions. We further discuss how one can match the lattice to experiment. In 3.3, we calculate the perturbative one-loop corrections to the quasidistribution as a necessary step in matching the lattice to experiment. The discussion of our results in 3.3 continues in 3.4, wherein we analyze the infrared differences between the one-loop quasidistribution corrections in Euclidean space vs. the same in Minkowski space. 3.5 ends this chapter with a discussion of recent work from collaborators which demonstrated a 
resolution to the infrared problems found within the one-loop corrections.

\subsection{Introduction to DIS and the quark distribution func- tion}

Hadrons, such as the protons and neutrons found within a given atom, are bound quark and gluon states. The internal properties of this bound state dictate the external behavior of each hadron just like the makeup of protons, neutrons, and electrons dictate the chemical behaviors of an atom. For example, in principle one can calculate the electromagnetic radius of the proton by calculating the low energy bound state wave function of the quarks and gluons. In practice, such calculations are quite difficult due to the non-perturbative nature of QCD at low energies. Of course, difficulties in theoretical calculations do not necessarily hold back experimentalists from experimenting.

One method experimentalists use to probe the inner structure of hadrons is deep inelastic scattering (DIS). Therein, a lepton beam is scattered off a hadronic target with sufficient four-momentum transfer $\left(Q^{2}>1 \mathrm{GeV}^{2}\right.$ to resolve the individual quarks (see Figure 3.1). From the momenta and directions of the outgoing lepton and hadrons, experimentalists can extract relevant information, such as the distribution of the proton's momentum across its constituent quarks and gluons. The corresponding DIS cross section, proportional to the process' amplitude squared, will also contain the distribution of quark and gluon momenta. DIS then offers both a test of QCD and an insight into its non-perturbative nature.

The DIS amplitude for a lepton $l$ scattering off a proton $p$ with momentum transfer $q$ resulting in an outgoing lepton $l^{\prime}$ and outgoing generalized hadronic state $X$ (to be summed over) is

$$
\mathcal{M}_{\mathrm{DIS}}=-\frac{e^{2}}{q^{2}}\left\langle l^{\prime}\left|j_{\mu}(0)\right| l\right\rangle\left\langle X\left|j^{\mu}(0)\right| p\right\rangle
$$

where $j_{\mu}(0)$ is the electromagnetic current

$$
j_{\mu}(0)=\bar{\psi}(0) \gamma_{\mu} \psi(0)
$$

for the lepton-lepton interaction and for the hadronic interaction (where, for now, we've ignored the constituent quark nature of the hadronic), and where $e$ is the electromagnetic charge of the lepton and proton. The DIS cross section observed in experiment is proportional to $\left|\mathcal{M}_{\mathrm{DIS}}\right|^{2}$. We now demonstrate how the squared amplitude can be written and massaged in such a way as to extract the distribution of quark momenta within it. 


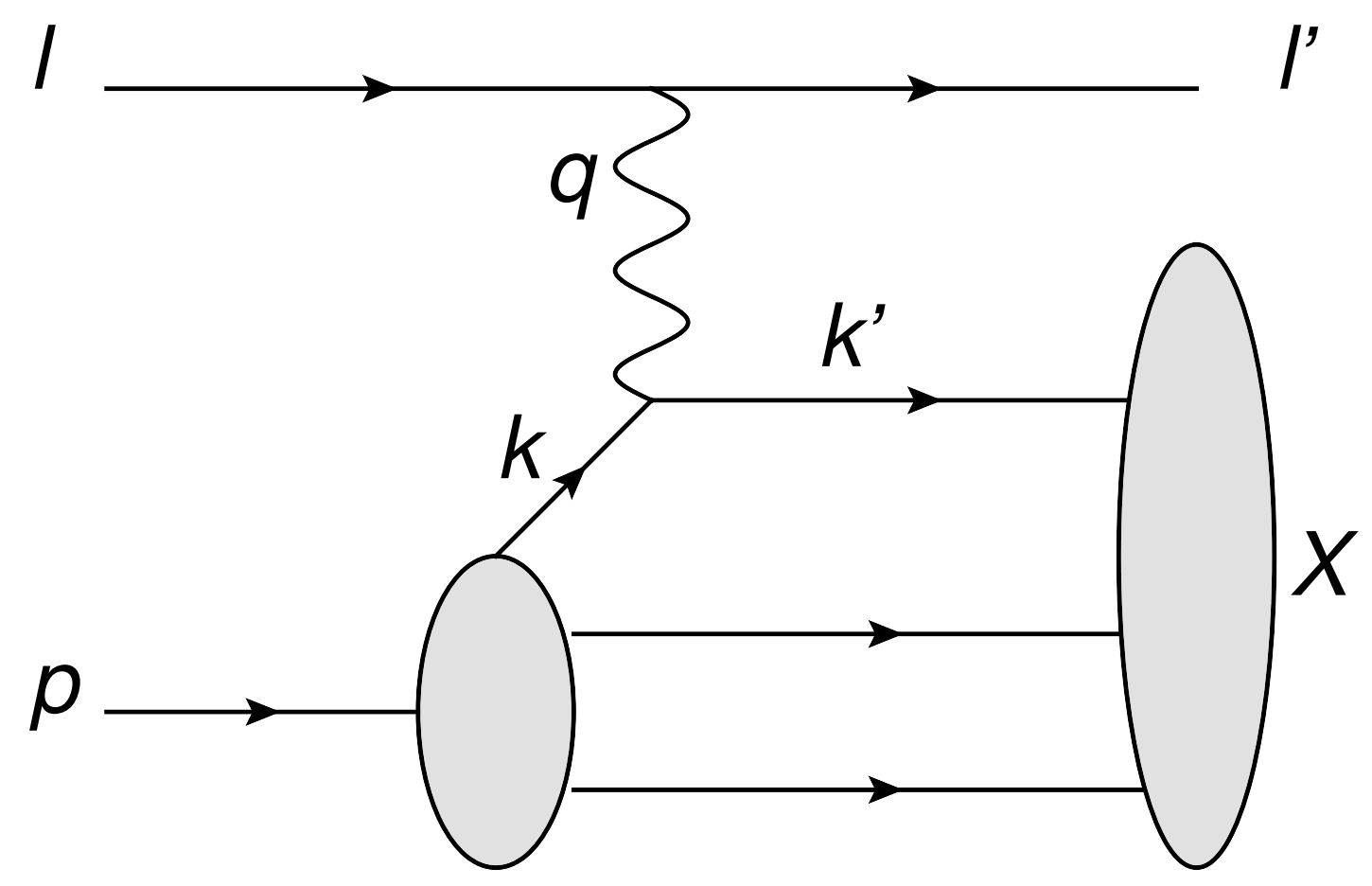

Figure 3.1: Deep Inelastic Scattering (DIS): a lepton with momentum $l$ is scattered off a proton with momentum $p$ resulting in a shower of hadronic states $X$. The inelasticity of DIS results from a sufficient energy transfer $q$, such that the lepton interacts with an individual quark of momentum $k$. In light-cone coordinates $k$ is a fraction of the proton's momentum $p$.

The squared amplitude is

$$
\left|\mathcal{M}_{\mathrm{DIS}}\right|^{2}=\left(\frac{e^{2}}{q^{2}}\right)^{2}\left\langle l\left|j_{\mu}(0)\right| l^{\prime}\right\rangle\left\langle l^{\prime}\left|j_{\nu}(0)\right| l\right\rangle\left\langle p\left|j^{\mu}(0)\right| X\right\rangle\left\langle X\left|j^{\nu}(0)\right| p\right\rangle .
$$

The internal structure of the hadron is probed within the hadronic part $\left\langle p\left|j^{\nu}(0)\right| X\right\rangle\left\langle X\left|j^{\mu}(0)\right| p\right\rangle$ of the DIS amplitude squared. As we are only interested in the structure of the incoming hadron, we sum over all external hadronic states $X$ and, since the cross section contains it, with momentum conservation imposed by $(2 \pi)^{4} \delta\left(q+p-p_{X}\right)$ where $p_{X}$ is the momentum of a given external hadronic state $X$. Normalizing by $4 \pi$, we write this hadronic part as the hadronic tensor $W^{\mu \nu}$

$$
W^{\mu \nu}=\frac{1}{4 \pi} \sum_{X}(2 \pi)^{4} \delta\left(q+p-p_{X}\right)\left\langle p\left|j^{\mu}(0)\right| X\right\rangle\left\langle X\left|j^{\nu}(0)\right| p\right\rangle .
$$

We would like the hadronic tensor written in such a way as to remove the dependence on the external states $X$. We accomplish this in two steps. One, we write the Dirac delta function in it's integral form,

$$
(2 \pi)^{4} \delta\left(q+p-p_{X}\right)=\int d^{4} y e^{i y\left(q+p-p_{X}\right)} .
$$


Two, we transpose the matrix element $\left\langle p\left|j^{\nu}(0)\right| X\right\rangle$ from 0 to $y$ via,

$$
\left\langle p\left|j^{\mu}(0)\right| X\right\rangle=e^{i y\left(p_{X}-p\right)}\left\langle p\left|j^{\mu}(y)\right| X\right\rangle .
$$

Put together, we have

$$
W^{\mu \nu}=\frac{1}{4 \pi} \sum_{X} \int d^{4} y e^{i q y}\left\langle p\left|j^{\mu}(y)\right| X\right\rangle\left\langle X\left|j^{\nu}(0)\right| p\right\rangle .
$$

Now that the summation no longer has $p_{X}$ dependence, we use can freely apply our identity operator

$$
\sum_{X}|X><X|=1
$$

to write

$$
W^{\mu \nu}=\frac{1}{4 \pi} \int d^{4} y e^{i q y}\left\langle p\left|j^{\nu}(y) j^{\mu}(0)\right| p\right\rangle .
$$

Though this form of the hadronic tensor now only depends on the structure of the incoming hadron, we have not yet implemented the constraint that within the standard DIS process, we consider only a single internal quark interacting with the incoming lepton. We thus need to extract this quark (seen in 3.1) of fractional charge $e_{i}$, momentum $k^{\prime}$ and spin $s^{\prime}$ from the external hadronic state $X$ in 3.7 by writing

$$
\left\langle p\left|j^{\nu}(y)\right| X\right\rangle=\left\langle p\left|e_{i} \bar{\psi}(y) \gamma^{\nu} \psi(y)\right| k^{\prime}, s^{\prime}, X^{\prime}\right\rangle=\left\langle p\left|e_{i} \bar{\psi}(y) \gamma^{\nu} e^{-i k^{\prime} y} u\left(k^{\prime}, s^{\prime}\right)\right| X^{\prime}\right\rangle
$$

where $X^{\prime}$ is a hadronic state equal to $X$ without a quark of momentum $k^{\prime}$ and spin $s^{\prime}$. Similarly we write

$$
\left\langle X\left|j^{\mu}(0)\right| p\right\rangle=\left\langle k^{\prime}, s^{\prime}, X^{\prime}\left|e_{i} \bar{\psi}(0) \gamma^{\mu} \psi(0)\right| p\right\rangle=\left\langle X^{\prime}\left|\bar{u}\left(k^{\prime}, s^{\prime}\right) \gamma^{\mu} \psi(y)\right| p\right\rangle .
$$

As before, we now sum over all $X^{\prime}$ states (which yields the identity) in addition to summing/integrating over all on-shell external quark states of momentum $k^{\prime}$, spin $s^{\prime}$, and flavor $i$ such that the hadronic tensor is

$$
\begin{aligned}
W^{\mu \nu} & =\frac{1}{4 \pi} \sum_{i} \sum_{s^{\prime}} \int d^{4} y \int \frac{d^{4} k^{\prime}}{(2 \pi)^{3}} \delta\left(k^{\prime 2}\right) e^{i\left(q-k^{\prime}\right) y}\left\langle p\left|e_{i}^{2} \bar{\psi}(y) \gamma^{\nu} u\left(k^{\prime}, s^{\prime}\right) \bar{u}\left(k^{\prime}, s^{\prime}\right) \gamma^{\mu} \psi(0)\right| p\right\rangle \\
& =\frac{1}{4 \pi} \sum_{i} e_{i}^{2} \int d^{4} y \int \frac{d^{4} k^{\prime}}{(2 \pi)^{3}} \delta\left(k^{\prime 2}\right) e^{i\left(q-k^{\prime}\right) y}\left\langle p\left|\bar{\psi}(y) \gamma^{\nu} \not k^{\prime \prime} \gamma^{\mu} \psi(0)\right| p\right\rangle
\end{aligned}
$$

Note that we have set the quark mass to zero and used the spin-sum identity. We further simplify the hadronic tensor by noting $k^{\prime}=k+q$ (see. Fig. 3.1) such that

$$
\begin{aligned}
W^{\mu \nu} & =\frac{1}{4 \pi} \sum_{i} e_{i}^{2} \int d^{4} y \int \frac{d^{4} k^{\prime}}{(2 \pi)^{3}} \delta\left((k+q)^{2}\right) e^{i\left(q-k^{\prime}\right) y}\left\langle p\left|\bar{\psi}(y) \gamma^{\nu} \not k^{\prime} \gamma^{\mu} \psi(0)\right| p\right\rangle \\
& =\frac{1}{4 \pi} \sum_{i} e_{i}^{2} \int d^{4} y \int \frac{d^{4} k}{(2 \pi)^{3}} \delta\left((k+q)^{2}\right) e^{-i k y}\left\langle p\left|\bar{\psi}(y) \gamma^{\nu}(\not k+\not q) \gamma^{\mu} \psi(0)\right| p\right\rangle
\end{aligned}
$$


where on the last step we've changed our integration variables to from $k^{\prime}$ to $k$ with the same measure.

A particularly useful simplification of $W^{\mu \nu}$ occurs when we view the hadronic tensor from a frame of infinite proton momentum. That is, we will boost the proton's momentum $p$ along an arbitrary direction, say $z$, to such an extent that we can remove components of momentum which become small under such a boost. These small components can be readily identified using light-cone coordinates, which simplifies such boosts. We now take a brief interlude to discuss light-cone coordinates.

In standard Cartesian coordinates, the proton has momentum $p=\left(p^{0}, p^{1}, p^{2}, p^{3}\right)$ where we identify $p^{0}$ as the energy of the proton and $p^{1,2,3}$ as the proton's 3 -momentum in $(\mathrm{x}, \mathrm{y}, \mathrm{z})$ space. In light-cone coordinates, we perform a change of variables such that $p=\left(p^{+}, p^{-}, \mathbf{p}^{T}\right)$ where

$$
\begin{aligned}
p^{+} & =\frac{p^{0}+p^{3}}{\sqrt{2}} \\
p^{-} & =\frac{p^{0}-p^{3}}{\sqrt{2}} \\
\mathbf{p}^{T} & =\left(p^{1}, p^{2}\right)
\end{aligned}
$$

Note the factors of $\sqrt{2}$ are purely convention. If we additionally choose a frame such that the proton and photon are only traveling in the $z$ direction with large (infinite) proton momentum

$$
p^{+} \gg p^{-},
$$

then the dot product between $p$ and, say $q$, is

$$
p \cdot q=p^{+} q^{-}+p^{-} q^{+}+\mathbf{p}^{T} \cdot \mathbf{q}^{T}=p^{+} q^{-},
$$

where we've set the photon energy $q^{0}=0$ such that

$$
q^{-}=-q^{+}
$$

This frame choice becomes particularly useful as its applied to our hadronic tensor below. Note, a more formal treatment of the $p^{+}$large behavior in the light-front formalism and the factorization of bound state physics out of scattering in QCD is available in Collin's "Foundations of Perturbative QCD" [61].

In this frame, the proton is moving fast enough such that time is dilated to the point where the internal interactions amongst the constituent quarks and gluons becomes frozen within the Lorentz contracted proton. 


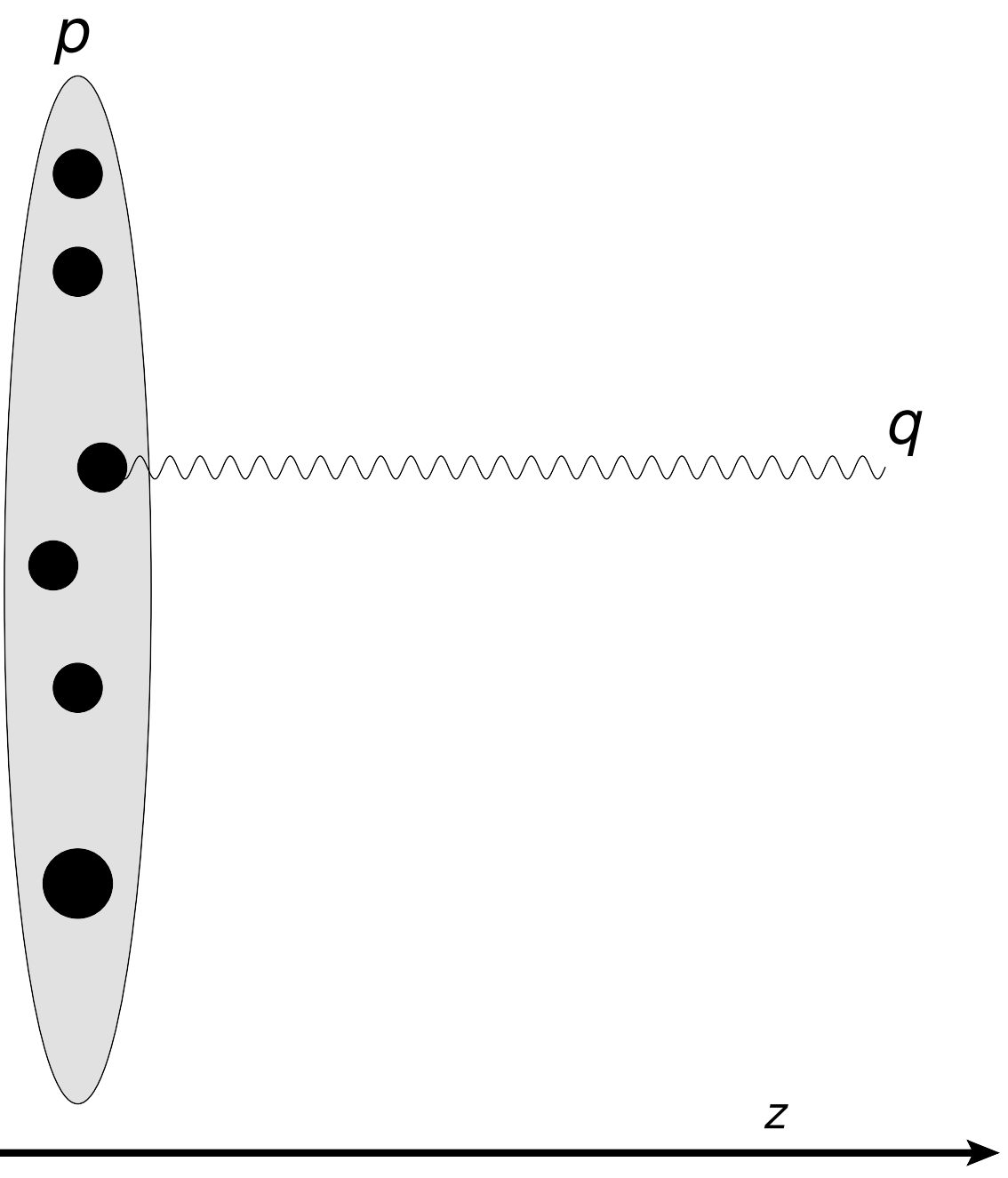

Figure 3.2: In the infinite momentum frame, the proton moving with very large momentum in the $z$ direcion becomes Lorentz contracted and time dilated. The constituent quarks and gluons become spread out and frozen. The photon probe with momentum $q$ also moving in he $z$ direction interacts with a free quark unaffected by low-energy bound-state interactions.

To picture this, consider the proton's radius of about 1 femtometer [62]. As the proton moves in the $z$ direction with large momentum, the proton's size is squished into a disk such that the constituent quarks and gluons become spaced out (see Fig. 3.2). Furthermore, within the proton's rest frame, the quarks and gluons interact with each other on a timescale of roughly 1 femtometer. However, in the lab frame where collisions between protons and electrons occur with center-of-mass energy in the hundreds of $\mathrm{GeV}$, the timescale of the proton's constituents becomes dilated by about 150 times [61].

This time-dilation implies that, at the moment at which the electron is scattered off the proton, the last constituent quark or gluon interaction occurred a long time and distance, about $100 \mathrm{fm}$, away from the electron scattering event. Thus we can ignore its effects. 


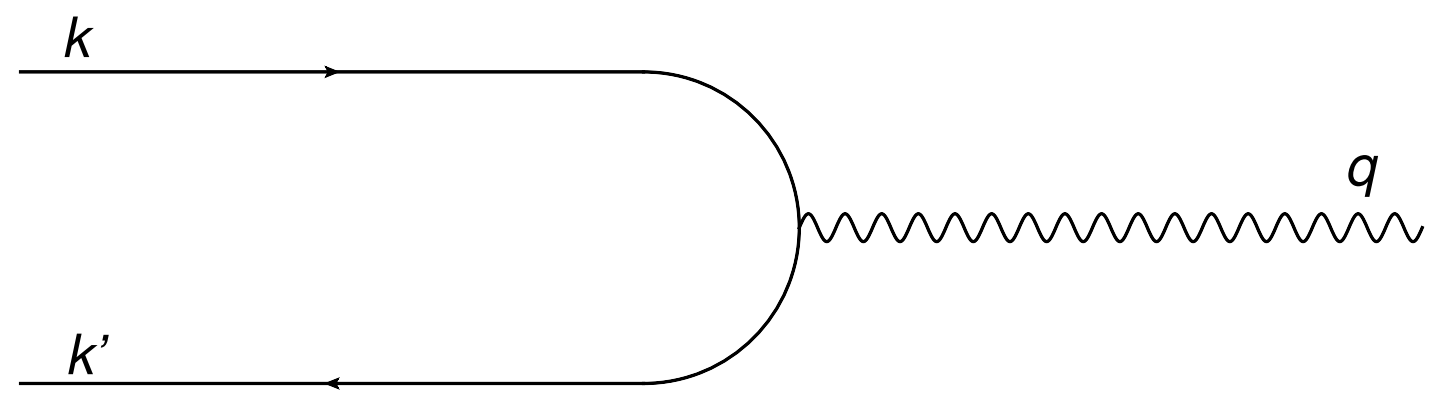

$Z$

Figure 3.3: An on-shell, zero mass, free constituent quark of momentum $k$ moving in the $z$ direction scatters off photon with momentum $q$ also moving in the $z$ direction, resulting in a quark of momentum $k^{\prime}$ with $k_{z}=-k_{z}^{\prime}$.

That is, these quarks and gluons, or "partons", become free, on-shell (with mass $m$, set to zero later on) particles moving in the same direction as their carrier proton. In fact, it is this insight that motivated Feynman's parton model [63] in which we can neglect the interactions which bind the quarks and gluon into baryons, within shortterm scattering events.

We now consider, in more detail, this frame where the proton and photon both move in the $z$ direction with large momentum $p^{+} \gg p^{-}$. The on-shell, zero mass, constituent quarks move with a fraction $x$ of the proton's momentum where the interaction between the incoming and outgoing quarks (with momentum $k$ and $k^{\prime}$ respectively) with the photon of momentum $q$ is shown side-by-side in Cartesian coordinates (Fig. 3.3) and lightcone coordinates (Fig. 3.4).

That is,

$$
\begin{aligned}
p & =\left(p^{+}, \frac{M^{2}}{2 p^{+}}, \mathbf{0}^{T}\right) \\
k & =\left(x p^{+}, 0, \mathbf{0}^{T}\right) \\
k^{\prime} & =\left(0, x p^{+}, \mathbf{0}^{T}\right) \\
q & =k^{\prime}-k=\left(-x p^{+}, x p^{+}, \mathbf{0}^{T}\right),
\end{aligned}
$$

were $M$ is the mass of the proton and

$$
x \equiv \frac{k^{+}}{p^{+}}
$$




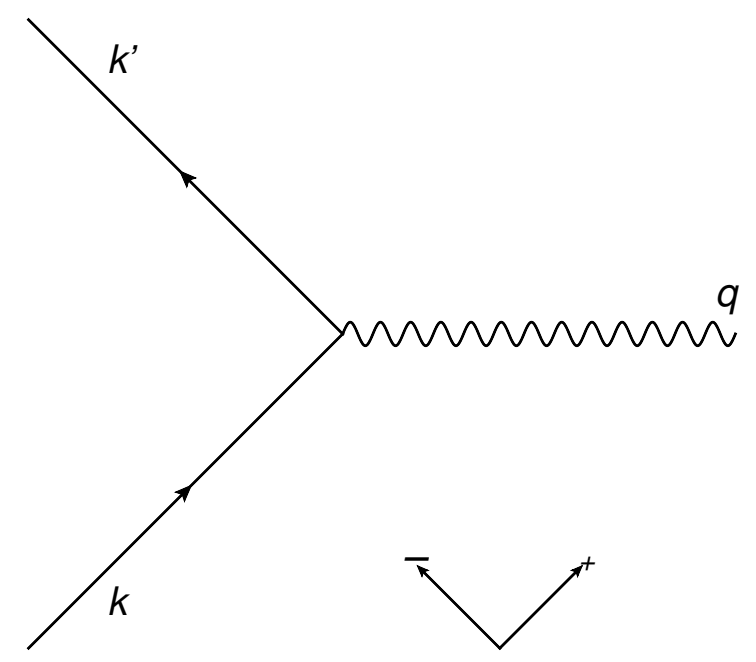

Figure 3.4: The lightcone coordinates analog of Fig. 3.3. An on-shell, zero mass, free constituent quark of momentum $k$ moving in the + direction scatters off photon with momentum $q$, resulting in a quark of momentum $k^{\prime}$ moving in the - direction such that $k^{+}=k^{-}$.

We now see,

$$
\begin{aligned}
& k^{+}=-q^{+} \\
& k^{-}=0 \\
& q^{-}=x p^{+} .
\end{aligned}
$$

Furthermore,

$$
\begin{aligned}
p \cdot q & =x\left(p^{+}\right)^{2}-x \frac{M^{2}}{2} \sim x\left(p^{+}\right)^{2} \\
& \rightarrow x=\frac{p \cdot q}{\left(p^{+}\right)^{2}} .
\end{aligned}
$$

It'll be useful down the line to write $p \cdot q$ in terms of the proton's mass and the photon's energy $\nu$ defined within the proton's rest frame,

$$
p \cdot q \equiv M \nu .
$$

Therefore,

$$
\begin{aligned}
x & =\frac{M \nu}{\left(p^{+}\right)^{2}} \\
q^{2} & =-2 x^{2}\left(p^{+}\right)^{2}=-2 x M \nu \equiv-Q^{2},
\end{aligned}
$$

and

$$
\begin{aligned}
x & =\frac{Q^{2}}{2 M \nu} \\
& =\frac{Q^{2}}{2 p \cdot q} .
\end{aligned}
$$


Thus, for large proton momentum $p^{+}$, we are able to write down the following identities:

$$
\begin{aligned}
\not k+q & =\gamma^{+} q^{-}=\gamma^{+} \frac{M \nu}{p^{+}} \\
(k+q)^{2} & =k^{2}+q^{2}+2 k \cdot q=-2 x M \nu+2 k^{+} q^{-}=2 M \nu\left(\frac{k^{+} q^{-}}{M \nu}-x\right) \\
& =2 M \nu\left(\frac{k^{+}}{p^{+}}-x\right),
\end{aligned}
$$

which simplify our hadronic tensor to

$$
\begin{aligned}
W^{\mu \nu} & =\frac{1}{8 \pi p^{+}} \int d^{4} y \sum_{i} e_{i}^{2} \int \frac{d^{4} k}{(2 \pi)^{3}} e^{-i k y} \delta\left(\frac{k^{+}}{p^{+}}-x\right)\left\langle p\left|\bar{\psi}(y) \gamma^{\mu} \gamma^{+} \gamma^{\nu} \psi(0)\right| p\right\rangle \\
& =\frac{1}{8 \pi} \int d^{4} y \delta\left(y^{+}\right) \delta\left(\mathbf{y}^{T}\right) e^{-i x p^{+} y^{-}} \sum_{i} e_{i}^{2}\left\langle p\left|\bar{\psi}(y) \gamma^{\mu} \gamma^{+} \gamma^{\nu} \psi(0)\right| p\right\rangle
\end{aligned}
$$

We now take a look at the $W^{22}$ diagonal element of the hadronic tensor, which we will find is particularly interesting.

$$
W^{22}=\frac{1}{8 \pi} \int d y^{-} e^{-i x p^{+} y^{-}} \sum_{i} e_{i}^{2}\left\langle p\left|\bar{\psi}\left(y^{-}\right) \gamma^{+} \psi(0)\right| p\right\rangle,
$$

where we've used

$$
\left\{\gamma^{\mu}, \gamma^{\nu}\right\}=2 g^{\mu \nu}
$$

We now identify within the hadronic tensor, the quark distribution function at some scale $\mu^{2}$ denoted $q\left(x, \mu^{2}, P^{+}\right)$and defined as

$$
q\left(x, \mu^{2}, P^{+}\right)=\int_{-\infty}^{\infty} \frac{d \xi^{-}}{4 \pi} e^{-i x \xi^{-} P^{+}}\left\langle P\left|\bar{\psi}\left(\xi^{-}\right) \gamma^{+} 1 \psi(0)\right| P\right\rangle
$$

where $x$, in light-cone coordinates, is the momentum of the constituent quark as a fraction of its encompassing proton's momentum. That is

$$
x=k^{+} / P^{+} .
$$

Note the isolated identity 1 in (3.40). We write this 1 explicitly as it is an artifact of our particular derivation. A more generalized derivation will result in this 1 replaced by a "gauge link" term of the form

$$
W\left(\xi^{-}\right)=\exp \left(-i g \int_{0}^{\xi^{-}} d \eta^{-} A^{+}\left(\eta^{-}\right)\right) .
$$

In the analysis below, we've implicitly chosen to work in light-front gauge $A^{+}=0$ where $A$ is the gluon field. This sets a gauge-link type interaction $W\left(\xi^{-}\right)$to identity, which simplifies one-loop corrections to the quark distribution function. 
The use of light-cone coordinates, which intermix time and space (see 3.14), in defining the quark distribution function causes a dilemma for the lattice. As stated earlier, the lattice is a numerical Euclidean approximation of the real continuum Minkowskian space-time. One rotates to Euclidean space-time from Minkowskian space-time through the standard Wick rotation

$$
t \rightarrow i t
$$

where $t$ is time. This rotation connects the time-evolution operator to statistical mechanics through

$$
e^{i t H} \rightarrow e^{-t H}
$$

A side effect working in light-cone coordinates is that the plus and minus components of space-time become complex after Wick rotating into Euclidean space. That is $\xi^{-}$of (3.40) becomes

$$
\xi^{-}=\frac{1}{\sqrt{2}}\left(\xi^{0}-\xi^{3}\right) \rightarrow \frac{1}{\sqrt{2}}\left(i \xi^{0}-\xi^{3}\right)
$$

Therefore, the $\xi^{-}$integral within quark distribution function becomes an integration over the complex plane. The lattice, being a numerical algorithm, cannot perform calculations over a complex plan.

Lattice theorists can overcome this problem by extracting the quark distribution function from its Mellin moments

$$
a_{n}=\int_{0}^{1} d x x^{n-1} q(x)
$$

where

$$
q(x)=\frac{1}{2 \pi i} \int_{-i \infty}^{+i \infty} d n x^{-n} a_{n}
$$

which can be written as a summation of poles within the contour.

The Mellin moments $a_{n}$ have a convenient feature which removes the complex inte- 
gration over $\xi^{-}$within the Wick-rotated quark distribution. That is,

$$
\begin{aligned}
a_{n} & =\int_{0}^{1} d x x^{n-1} \int_{-\infty}^{\infty} \frac{d \xi^{-}}{4 \pi} e^{-i x \xi^{-} P^{+}}\left\langle P\left|\bar{\psi}\left(\xi^{-}\right) \gamma^{+} \psi(0)\right| P\right\rangle \\
& =\int_{0}^{1} d x x^{n-1} \int_{-\infty}^{\infty} \frac{d \xi^{-}}{4 \pi} e^{i x \xi^{-} P^{+}}\left\langle P\left|\bar{\psi}(0) \gamma^{+} \psi\left(\xi^{-}\right)\right| P\right\rangle \\
& =\int_{0}^{1} d x \int_{-\infty}^{\infty} \frac{d \xi^{-}}{4 \pi}\left[\left(\frac{d}{d\left(\mathrm{i} \xi^{-} P^{+}\right)}\right)^{n-1} e^{i x \xi^{-} P^{+}}\right]\left\langle P\left|\bar{\psi}(0) \gamma^{+} \psi\left(\xi^{-}\right)\right| P\right\rangle \\
& =\left(\frac{1}{P^{+}}\right)^{n-1} \int_{0}^{1} d x \int_{-\infty}^{\infty} \frac{d \xi^{-}}{4 \pi}\left[\left(\frac{-i d}{d \xi^{-}}\right)^{n-1} e^{i x \xi^{-} P^{+}}\right]\left\langle P\left|\bar{\psi}(0) \gamma^{+} \psi\left(\xi^{-}\right)\right| P\right\rangle \\
& =\left(\frac{1}{P^{+}}\right)^{n-1} \int_{0}^{1} d x \int_{-\infty}^{\infty} \frac{d \xi^{-}}{4 \pi} e^{i x \xi^{-} P^{+}}\left\langle P\left|\bar{\psi}(0) \gamma^{+}\left[\left(\frac{i d}{d \xi^{-}}\right)^{n-1} \psi\left(\xi^{-}\right)\right]\right| P\right\rangle,
\end{aligned}
$$

where, on the last step, we've performed an integration by parts, noting the surface term on $\xi^{-}$goes to zero.

We now note that the integrand on $x$ is only the exponential $e^{i x \psi^{-} P^{+}}$. Therefore,

$$
\begin{aligned}
a_{n} & =\left(\frac{1}{P^{+}}\right)^{n-1} \int_{-\infty}^{\infty} \frac{d \xi^{-}}{2} \delta\left(\xi^{-} P^{+}\right)\left\langle P\left|\bar{\psi}(0) \gamma^{+}\left[\left(\frac{i d}{d \xi^{-}}\right)^{n-1} \psi\left(\xi^{-}\right)\right]\right| P\right\rangle \\
& =\left(\frac{1}{P^{+}}\right)^{n} \frac{1}{2}\left\langle P\left|\bar{\psi}(0) \gamma^{+}\left[\left(\frac{i d}{d \xi^{-}}\right)^{n-1} \psi(0)\right]\right| P\right\rangle,
\end{aligned}
$$

where we now have the very convenient result

$$
\left\langle P\left|\bar{\psi}(0) \gamma^{+}\left[\left(i \partial^{+}\right)^{n-1} \psi(0)\right]\right| P\right\rangle=2 a_{n}\left(P^{+}\right)^{n},
$$

from which we can write down the generalized result

$$
\left\langle P\left|\bar{\psi}(0) \gamma^{\mu_{1}} \partial^{\mu_{2}} \ldots \partial^{\mu_{n}} \psi(0)\right| P\right\rangle \sim 2 a_{n} P^{\mu_{1}} \ldots P^{\mu_{n}}
$$

where

$$
\partial^{+} \equiv \frac{\partial}{\partial \xi^{-}}
$$

Thus, we can obtain the Mellin moments $a_{n}$ from the purely real matrix element

$$
\left\langle P\left|\bar{\psi}(0) \gamma^{z}\left(\partial^{z}\right)^{n} \psi(0)\right| P\right\rangle=2 a_{n}\left(P^{z}\right)^{n}
$$

where the left-hand-side can be calculated on the lattice for small $n$.

There are two limitations with this procedure. One, a sufficient number of Mellin moments must be calculated to recreate the distribution function (see (3.47)). And two, sufficient precision must be obtained in the calculation for each Mellin moment. Both of these limitations remain difficult hurdles to overcome. 
A more recent technique to extract PDFs from the lattice has been developed by X. Ji and collaborators $[64,65,66,67,68,69,70,71,72]$. In this method, one takes a large-momentum limit of the quark distribution function from the onset. That is

$$
\begin{aligned}
q\left(x, \Lambda^{2}, P^{z}\right)= & \int_{-\infty}^{\infty} \frac{d z}{4 \pi} e^{i x z P^{z}}\left\langle P\left|\bar{\psi}(z) \gamma^{z} W(z) \psi(0)\right| P\right\rangle \\
& +\mathcal{O}\left(\Lambda^{2} /\left(P^{z}\right)^{2}, M^{2} /\left(P^{z}\right)^{2}\right)
\end{aligned}
$$

where $\Lambda$ is the QCD "scale parameter" $\Lambda_{\mathrm{QCD}}$ (see Section 1.2).

The leading order contribution to $q(x)$ in this limit is defined as the quark quasidistribution

$$
\tilde{q}(x)=\int_{-\infty}^{\infty} \frac{d z}{4 \pi} e^{i x z P^{z}}\left\langle P\left|\bar{\psi}(z) \gamma^{z} W(z) \psi(0)\right| P\right\rangle
$$

The quasidistribution, being independent of time, can be directly calculated on the lattice. Note that the gauge link $W(z)$ can be taken to identity as in the physical quark distribution function by working in Axial gauge $\left(A^{z}=0\right)$.

\subsection{The matching process and an introduction to LPT}

Lattice gauge theory is a space-time discretization of quantum field theory. Scattering amplitudes on the lattice are intrinsically regulated at all orders through the space-time lattice spacing $a$ which introduces an ultraviolet momentum cutoff $1 / a$. Long-range correlations are ignored by placing the lattice on finite-sized hypercube of length $L$.

The granularity of space-time removes the Lorentz symmetries embedded within continuum quantum field theory. But, gauge invariance and internal symmetries can be maintained. This allows the lattice gauge theory to be a fully renormalizable theory which can be written down as discretized analogs of electroweak and the strong interactions.

The numerical discretization of space-time allows the low-energy nonperturbative nature of QCD to be studied computationally. Successes from lattice calculations include the demonstration of confinement within the strong-coupling (long-distance) regime, an understanding of the significant contribution to hadronic mass, and hadronic spin from gluon interactions (as opposed to constituent quark interactions).

Nevertheless, there are inherent limitations on the power of lattice gauge theory. Namely, one most extrapolate to the continuum in order to correspond lattice predictions with physical observables. That is, though the lattice can calculate its version of the quark quasidistribution for a given hadron, this is not the quantity that experimentalists measure. 
In particular, we have no way of extracting out regularization dependence (as a result of space-time discretization) directly from the lattice. Therefore, we cannot match lattice predictions directly to experiment. Fortunately, we can gain an understanding of lattice regularization within the weak-coupling, perturbative, regime.

Within the high-energy regime, we can safely apply perturbative theory to lattice gauge theory, and, in doing so, derive a matching equation which replaces lattice regularization with more standard continuum-based schemes such as MS bar. This is forms the context of our work: match lattice perturbation theory (LPT) to continuum-based renormalization schemes for a given quantity (in this case, the quark quasidistribution).

So far we have only considered the discretization differences between the lattice and the continuum, and have ignored the Euclidean nature of the lattice as opposed to the Minkowskian nature of real spacetime. Our expectation in this neglect is to find no differences between the Euclidean and Minkowskian quasidistributions in the high-energy perturbative regime of the quasidistribution. Though these artifacts could be corrected for at a given order in perturbation theory, they point to problems in the non-perturbative regime which cannot be corrected for. We can then summarize the matching process in the following steps.

Step one: Remove the lattice-based $1 / a$ UV divergences in $\tilde{q}(x)_{\text {Lat. }}$, the lattice equivalent of our quark quasidistribution (see (3.60)) at the order of interest in perturbation theory.

Step two: Add back the continuum-based UV divergences as they exist in $\tilde{q}(x)_{\text {Mink. }}$ for a given scheme.

Step three: After applying steps one and two, confirm $\tilde{q}(x)_{\text {Lat. }}=\tilde{q}(x)_{\text {Mink. }}$ up to removable discretization based terms.

At one loop, this matching process is summarized in the following equation:

$$
\begin{aligned}
q(x, \Lambda)= & \tilde{q}(x)\left(1+Z_{F}(\Lambda)-\tilde{Z}_{F}(\Lambda)\right) \\
& +\int_{x}^{1} \frac{d y}{y}\left(q^{(1)}(x / y, \Lambda)-\tilde{q}^{(1)}(x / y, \Lambda)\right) q(y, \Lambda)+\mathcal{O}\left(\alpha_{s}^{2}\right),
\end{aligned}
$$

where $\Lambda$ is an imposed momentum cutoff necessary to regulate the ultraviolet divergences within.

Here $Z_{F}\left(\tilde{Z}_{F}\right)$ is the wave function renormalization for the quark (quasi)distribution function at one-loop. Similarly $q^{(1)}(x / y, \Lambda)$ is the one-loop vertex correction. In general (that is arbitrary gauge) we then have four diagrams 3.5 to calculate to obtain the required matching. 


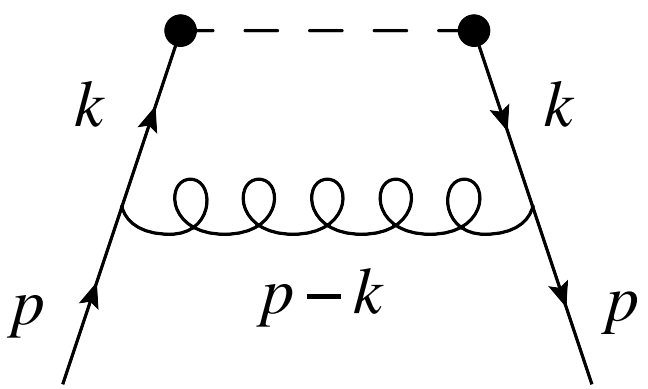

(a)

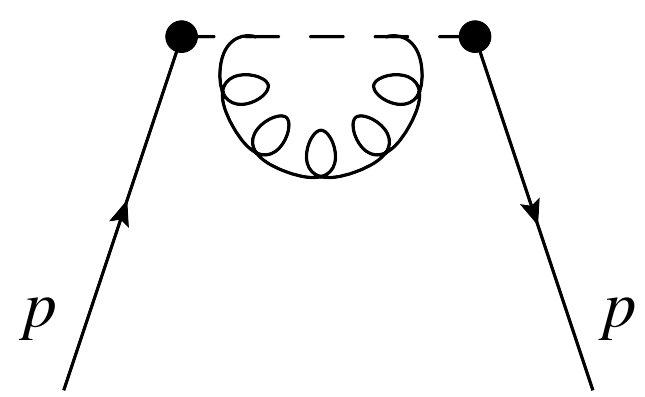

(c)

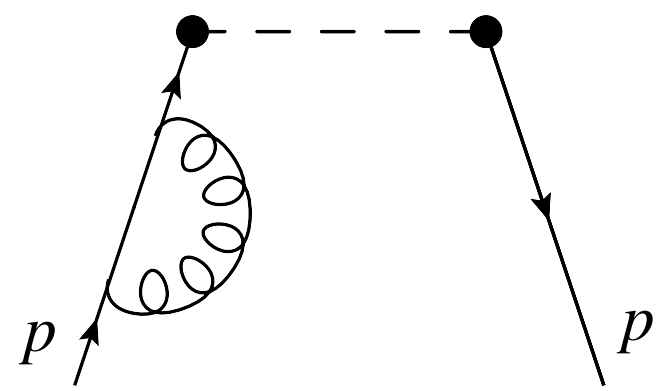

(b)

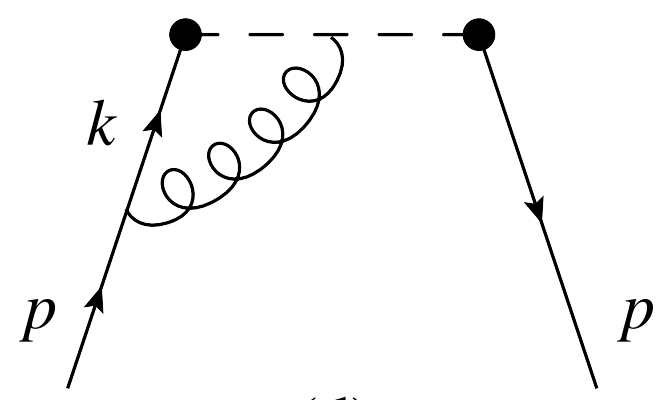

(d)

Figure 3.5: The one-loop corrections to the quark distribution function. Immediately, one notes that diagrams (c) and (d) can be gauged away by choosing light-front (or Axial in the case of the quasidistribution) gauge. Diagram (a) is the vertex correction. And diagram (b) is the wave-function renormalization.

With what needs to be calculated now clear, we move on to detail the perturbative and analytically theory which provides us the tools to do so: LPT.

First, we must Euclideanize the theory of QCD. In Euclidean space, we replace the Minkowskian metric $g_{\mu \nu}$, with signature $(1,-1,-1,-1)$, for the Euclidean metric $\delta_{\mu \nu}$, with signature $(1,1,1,1)$. Therefore, the Dirac matrices in Euclidean space, denoted $\gamma_{\mu}^{E}$, now satisfy our Euclidean anticommutation relation

$$
\left\{\gamma_{\mu}^{E}, \gamma_{\nu}^{E}\right\}=2 \delta_{\mu \nu}
$$

From the anticommutation relation, we identify

$$
\begin{aligned}
& \gamma_{0}^{E}=\gamma_{0}^{M} \\
& \gamma_{i}^{E}=-i \gamma_{i}^{M},
\end{aligned}
$$

where the subscript $i$ runs from one to three and $M$ denotes our Minkowskian gamma matrices which satisfy the standard $g_{\mu \nu}$ anticommutation relation. Note, throughout the rest of this chapter, we will write the Euclidean gamma matrices without their superscript $E$. 
Based on the Minkowskian QCD Lagrangian (see (1.68)), the Euclidean QCD Lagrangian (for one quark of mass $m$ ) is then

$$
\mathcal{L}_{\mathrm{QCD}}^{E}=\bar{\psi}(\not D+m) \psi(x)+\frac{1}{4} G_{\mu \nu}^{a} G_{\mu \nu}^{a}
$$

where we've pulled out an inconsequential overall minus sign and where repeated indices are summed over and contracted with the metric $\delta_{\mu \nu}$ (in Euclidean space, we will maintain the convention of keeping all repeated indices lowered).

The continuum Euclidean QCD action is

$$
S^{E}=\int d^{4} x \mathcal{L}_{\mathrm{QCD}}^{E}
$$

We wish to place this action onto a symmetric hypercube and discretize with lattice spacing $a$ and infinite volume (N.B. the lattice, as a computational method, places QCD on a finite-volume hypercube - this creates finite-volume-based artifacts, which can be corrected for, and which are outside the scope of this paper). Continuum-based integrals then become summations over lattice points $x$ where $x$ can take on only discrete values $n a$ where $n$ is an integer. In momentum space, the lattice spacing $a$ places an upper bound $\pi / a$ on the absolute value of a given particle's momentum. That is

$$
\begin{aligned}
& \int_{-\infty}^{\infty} d^{4} x \rightarrow a^{4} \sum_{x=a n} \\
& \int_{-\infty}^{\infty} d^{4} k \rightarrow \int_{-\pi / a}^{\pi / a} d^{4} k
\end{aligned}
$$

We are now ready discretize the Euclidean QCD action. There are a plethora of discretized actions which will reduce correctly to the Euclidean QCD action, in the continuum limit, as the lattice spacing $a$ is taken to zero. In this work, we will use the well-known, and relatively simple, Wilson action, so named after its inventor [73, 74]. Typical lattice calculations use the Wilson action, and so it becomes a convenient action to use LPT in order to match lattice observables with physical meaning.

The Wilson action, for one quark of mass $m$, is

$$
\begin{aligned}
S^{W}=a^{4} \sum_{x=a n}\{- & \frac{1}{2 a}\left[\bar{\psi}(x)\left(r-\gamma_{\mu}\right) U_{\mu}(x) \psi(x+a \hat{\mu})\right. \\
& \left.+\bar{\psi}(x+a \hat{\mu})\left(r+\gamma_{\mu}\right) U_{\mu}^{\dagger}(x) \psi(x)\right] \\
+ & \bar{\psi}(x)\left(m+\frac{4 r}{a}\right) \psi(x) \\
+ & \frac{1}{g_{s}^{2}}\left[3-\operatorname{Re} \operatorname{Tr}\left[U_{\mu}(x) U_{\nu}(x+a \hat{\mu}) U_{\mu}^{\dagger}(x+a \hat{\nu}) U_{\nu}^{\dagger}(x)\right]\right\},
\end{aligned}
$$


where, as before, repeated indices are summed over, and where the Wilson parameter $r$ is $0<r \leq 1$.

The first three terms in the Wilson action are the standard kinetic terms, with the derivatives of the quark fields are written out as a symmetric averaging of the quark fields between two lattice points, normalized by the lattice spacing. The lattice fields $U_{\mu}$ replace the continuum gluon gauge fields $G_{\mu}$ written down in 1.2 through a carefully chosen gauge with $\alpha^{a}(x)=-g_{s} a G_{\mu}^{a}(x)$. That is,

$$
U_{\mu}(x)=e^{i g_{s} a T^{a} G_{\mu}^{a}(x)} .
$$

This explicit insertion of the gauge fields which now depend on the lattice spacing $a$ guarantees the gauge invariance of the Wilson action for any $a$. The last term of the Wilson action we then identify as an analog of the typical SU(3) field-strength tensors to all orders in the lattice spacing. The zero'th order of the expansion is simply the trace of the identity matrix, which we cancel out through the explicit factor of 3 .

For a full discussion and review of the LPT and the Wilson Action see Capitani's thorough review [75].

Within the context of this chapter, we will worker at first-order in the lattice spacing $a$ such that

$$
U_{\mu}(x) \sim 1+i g_{s} a T^{a} G_{\mu}^{a}(x),
$$

where we are able to work with the standard continuum-like gauge fields $G_{\mu}^{a}$.

From (3.69) we derive the Feynman rules necessary to calculate (3.61), that is all the diagrams in 3.5 .

$$
\begin{aligned}
S(k) & =\frac{1}{i \sum_{\mu} \gamma_{\mu} \sin a k_{\mu}+a m_{0}+2 r \sum_{\mu} \sin ^{2}\left(a k_{\mu} / 2\right)} \\
G_{\rho \lambda}(l) & =\frac{1}{\hat{l}_{\mu}^{2}}\left(\delta_{\rho \lambda}-\frac{\hat{l}_{\rho} \hat{n}_{\lambda}+\hat{n}_{\rho} \hat{l}_{\lambda}}{\hat{n} \cdot \hat{l}}+\frac{\hat{l}_{\rho} \hat{l}_{\lambda}}{(\hat{n} \cdot \hat{l})^{2}}\right) \\
\hat{l}_{\rho} & =\frac{2}{a} \sin \left(a l_{\rho} / 2\right), \\
\hat{n}_{\rho} & =n_{\mu} \cos \left(a l_{\rho} / 2\right), \\
V_{\rho}(p, k) & =i \gamma_{\rho} \cos \left(a(p+k)_{\rho} / 2\right)+r \sin \left(a(p+k)_{\rho} / 2\right)
\end{aligned}
$$

In $(3.72) S(k)$ is the Fermion propagator with momentum $k, G_{\rho \lambda}(l)$ is the photon propagator with momentum $l$, and $V_{\rho}(p, k)$ is the fermion-fermion-photon vertex where $p$ and $k$ are the incoming momenta. Discretization effects arise from the non-zero 
lattice spacing $a$. Additional LPT artifacts may occur from the Wilson parameter $r$ which accounts for fermion doubling on the lattice (see [75] for a full review). A quick check shows that one derives the continuum limit by taking $a$ to zero.

For a given process, one can use LPT to analytically calculate the UV behavior through standard Feynman diagram techniques. In this way, we analytically correct for the UV divergences in $\tilde{q}(x)_{\text {Lat. }}$ at a given order in $\alpha_{s}$.

We now have all the tools necessary to match the UV regime of the quark quasidistribution on the lattice to the UV regime of the quark distribution on the continuum.

\subsection{The One-Loop Corrections}

In the perturbative regime, at one-loop, there are four diagrams (Figure 3.5), denoted: (a), (b), (c), and (d), which contribute to the quark quasidistribution (3.60) vertex correction. In Axial gauge $A^{z}=0$, the gauge link $W(z)$ becomes identity and diagrams (b) and (d) do not contribute. Furthermore, one can use quark number conservation

$$
\int_{-\infty}^{\infty} d x \tilde{q}(x)=\int_{0}^{1} d x q(x)=1
$$

to solve for diagram (d), the wave function renormalization $Z_{F}$, in terms of diagram (a), the vertex correction $q^{(1)}$. Thus only diagram (a), the vertex correction, requires an explicit analytic calculation at one-loop.

The one-loop vertex correction to the quark quasidistribution in continuum Minkowski space is

$$
\begin{aligned}
& \tilde{q}_{\mathrm{M}}^{(1)}\left(x, \Lambda, p^{z}\right)=\frac{\alpha_{s} C_{F}}{2 \pi} \\
& \quad \times\left\{\frac{1+x^{2}}{1-x} \ln \frac{4 x p_{z}^{2}}{(1-x) m^{2}}-\frac{4 x}{1-x}+1+\frac{\Lambda}{(1-x)^{2} p^{z}}\right\}
\end{aligned}
$$

for momentum fraction $x$ between 0 and $1 . \Lambda$ is a momentum cutoff and $p^{z}$ is the momentum of the quark such that $x=p^{z} / P^{z}$ where $P^{z}$ is the proton's momentum, taken to be large. $C_{F}=4 / 3$ is the standard color factor.

Immediately evident in (3.74) is the infrared divergence as the quark mass $m$ is taken to zero. This arises when the momenta of the virtual quark and photon become collinear (for a review see $[76,77]$ ). If one were to calculate the vertex correction for the physical quark distribution (3.74), the same form of the logarithmic infrared divergence appears and so one cannot assume this behavior to be an artifact of the large $P^{z}$ limit. N.B. a careful observer might note that the ultraviolet term does differ between the 
physical and quasi one-loop corrections - such terms can be corrected for perturbatively and thus do not contribute to a physical difference.

$$
\begin{aligned}
q^{(1)}(x, \Lambda) & =\frac{\alpha_{s} C_{F}}{2 \pi} \\
\times & \left\{\frac{1+x^{2}}{1-x} \ln \frac{\Lambda^{2}}{(1-x)^{2} m^{2}}-\frac{2 x}{1-x}-\delta_{\epsilon}(1-x)\right\}
\end{aligned}
$$

We are interested in the lattice (that is the LPT) form of (3.74). However, one immediatly encounters some difficulties. First and foremost, the LPT Feynman rules (3.72) are trigonometric. This creates difficulties in analytically evaluating the loop integrals encountered in Fig. 3.5. To achieve the desired analytic result, we will expand the LPT Feynman rules around $a=0$ as shown in (3.76). The leading order terms will be, of course, the continuum Euclidean result.

$$
\begin{aligned}
& S= \frac{\left(-i \not k+m+\frac{a}{2} k^{2}\right)}{k^{2}+m^{2}}\left(1-a m \frac{k^{2}}{k^{2}+m^{2}}\right) \\
& G_{\rho \lambda}= \frac{1}{(p-k)^{2}}\left(\delta_{\rho \lambda}+\frac{(p-k)_{\rho}(p-k)_{\lambda}}{\left(p_{z}-k_{z}\right)^{2}}\right. \\
&\left.\quad-\frac{\delta_{\rho z}(p-k)_{\lambda}+\delta_{\lambda z}(p-k)_{\rho}}{p_{z}-k_{z}}\right) \\
& V_{\rho}=i \gamma_{\rho}+\frac{a}{2}(p+k)_{\rho}
\end{aligned}
$$

The careful reader may now become dubious for there will be a point, soon to arrive, where we will integrate over the loop-momentum $k$. Part of the region of integration occurs when $k$ is larger than $a$, thus making the small $a$ expansion invalid. Conveniently, all terms resulting from this integration except those which contain an ultraviolet divergence, must be small as $k$ becomes large. Thus, we will stick to this leading order expansion to extract the analytic finite terms. For the ultraviolet term, we will have to be more careful.

One must also ascertain whether $a p^{z}$ is small. In principle, $p^{z}$ can be taken as large as $P^{z}$ in the large-momentum limit where $P^{z}$ is tending towards infinity. However, in practice, that is on the lattice, $P^{z}$ is bounded by the discretization of space-time. Spacetime being no longer a continuum bounds the Fourier transform momentum space into a hypercube of width $\pi / a$. Thus, the largest $p^{z}$ can be is $\pi / a$, making $a p^{z}$ no longer a small quantity. We are forced then, to require

$$
P^{z}<1 / a
$$


such that $a p^{z}$ remains small.

Unfortunately, this constraint also places a bound on the momentum cutoff regulator $\Lambda$ in the vertex correction $\tilde{q}^{(1)}\left(x, \Lambda, p^{z}\right)$. As seen in (3.60), $P^{z}>\Lambda$ - this is what we mean by large momentum. Yet, on the lattice, we do not have free choice over our momentum cutoff. Phase space is explicitly bounded by $\pi / a$ and so we expect $\Lambda$ to go like $1 / a$. The consequences of these two constraints, the small lattice spacing limit, and the large momentum limit, being in conflict have been discussed by Radyushkin in $[78,79,80]$.

At leading order in $a$, the LPT Feynman rules reduce down to the Euclidean continuum result. If we ignore the LPT ultraviolet regime, for now, and assume a standard $\Lambda$ momentum cutoff, this Euclidean vertex correction is

$$
\begin{aligned}
\tilde{q}_{\mathrm{E}}^{(1)}\left(x, \Lambda, p_{z}\right)=\frac{-i g_{0}^{2} C_{F}}{4 \pi} \int \frac{d^{3} k}{(2 \pi)^{3}} \\
\times \frac{\bar{u}(p) \gamma_{\mu}(-i \not k+m) \gamma_{z}(-i \not k+m) \gamma_{\nu} u(p) d_{\mu \nu}(p-k)}{\left(k^{2}+m^{2}\right)^{2}(p-k)^{2}},
\end{aligned}
$$

where

$$
d_{\mu \nu}(q)=\delta_{\mu \nu}-\frac{q_{\mu} n_{\nu}+n_{\mu} q_{\nu}}{n \cdot q}+\frac{q_{\mu} q_{\nu}}{(n \cdot q)^{2}}
$$

with $n$ being a unit vector in the $z$-direction. The integration measure $d^{3} k=d k_{1} d k_{2} d k_{4}$, and $k_{3}=k_{z}=x p_{z}$. Note the phase space integrals here are over an infinite region where, later, we will introduce a momentum cutoff to regulate the result when necessary.

After careful work, we arrive at the final result, at leading order in the quark mass $m$ and quoted for all $x$ :

$$
\tilde{q}_{\mathrm{E}}^{(1)}=\frac{\alpha_{s} C_{F}}{2 \pi} \begin{cases}\frac{1+x^{2}}{1-x} \ln 2-\frac{1}{(1-x)^{2}}+2+\frac{\Lambda}{p_{z}(1-x)^{2}}, & 0<x<2, \\ \frac{2 x}{1-x} \ln \frac{x-1}{x}-1+\frac{\Lambda}{p_{z}(1-x)^{2}}, & x<0, \\ \frac{2 x}{1-x} \ln \frac{x}{x-1}-1+\frac{\Lambda}{p_{z}(1-x)^{2}}, & x>2,\end{cases}
$$

N.B. In this work we have placed the incoming and outgoing quarks onto their Euclidean mass shell (i.e. $p^{2}=-m^{2}$ ). In Section 3.5 we will discuss this assumption more carefully. For now, it is safe to say that whether the quark is placed on shell with positive mass, or negative mass, makes no difference in the analytic behavior of (3.80).

Furthermore, an astute observer will note the discontinuity in the regions around $x=0$ and $x=2$ in (3.80). This results from the small quark mass $m$ expansion. To reiterate, we worked "at leading order in the quark mass $m$." However, when $x$ (or $x-2$ ) become small numbers, this expansion is not valid. One can work out the general 
result, for all $m$, though we do not quote this here as it doesn't provide any additional insight into the analytic behavior, nor is it necessary to correct for the UV regime which is our overall goal.

In arriving at (3.80) from (3.78), one encounters the following loop-momentum integrals evaluated at $k_{z}=x p_{z}$ and which we now quote for reference later on:

$$
\begin{aligned}
& I_{1}=\int \frac{d^{3} k}{(2 \pi)^{3}} \frac{1}{\left(k^{2}+m^{2}\right)(p-k)^{2}}=\frac{\ln 2}{8 \pi p_{z}}, \\
& I_{2}=\int \frac{d^{3} k}{(2 \pi)^{3}} \frac{1}{\left(k^{2}+m^{2}\right)^{2}}=\frac{1}{8 \pi x p_{z}}, \\
& I_{3}=\int \frac{d^{3} k}{(2 \pi)^{3}} \frac{1}{\left(k^{2}+m^{2}\right)^{2}(p-k)^{2}}=-\frac{1}{32 \pi p_{z}^{3}} \frac{1-x}{x}, \\
& I_{4}=\int \frac{d^{3} k}{(2 \pi)^{3}} \frac{p \cdot k}{\left(k^{2}+m^{2}\right)(p-k)^{2}}=-\frac{(1-x) p_{z}}{8 \pi}, \\
& I_{5}=\int \frac{d^{3} k}{(2 \pi)^{3}} \frac{1}{(p-k)^{2}}=\frac{1}{4 \pi}\left(\Lambda-p_{z}\right) .
\end{aligned}
$$

N.B. $\Lambda$ is a momentum cutoff in spherical coordinates. This will be relevant later on as the explicit lattice cutoff $\pi / a$ is defined in Cartesian coordinates. Of these integrals, most notable is $I_{1}$ for it is in $I_{1}$ that we expect a logarithmic infrared divergence, but instead we find we are left with a finite $\ln (2)$. More explicitly, consider the Minkowskian equivalent of $I_{1}$, denoted $I_{1 M}$ :

$$
\begin{aligned}
I_{1 M} & \equiv-i \int \frac{d^{3} k}{(2 \pi)^{3}} \frac{1}{\left(k^{2}-m^{2}+i \epsilon\right)\left((p-k)^{2}+i \epsilon\right)} \\
& =\frac{1}{8 \pi p^{z}} \ln \frac{4 x\left(p^{z}\right)^{2}}{(1-x) m^{2}} .
\end{aligned}
$$

Though we are only interested in correcting the UV regime, finding now a difference in the regime where we expected both results to be the same is a dilemma we cannot ignore. LPT is only valid in the high energy region, when $\alpha_{s}$ is small. Thus, we cannot correct for this infrared difference. Of course, one could argue that the difference is found through an LPT calculation, which itself is not valid in the region where the difference occurs, and thus one asks: is there really any worry? That is, is there really a fundamental difference in the numerical quark quasidistribution as it is calculated from the lattice when compared to the experimental equivalent? Colleagues [60] have addressed these questions explicitly and we will discuss their work in 3.5. For now, we continue our work in the UV regime, and set aside the discrepancies discovered above.

As previously mentioned, the small $a$ expansion used in (3.76) is not valid in the UV regime. Thus, if we are interested in the correspondence between $\Lambda$ on the continuum 
and 1/a in LPT, we must return to our unexpanded Feynman rules (3.72) for the UV divergent integral $I_{5}$ in (3.81). This integral in LPT is

$$
\begin{array}{r}
I_{5 L}=\int_{-\pi / a}^{\pi / a} \frac{d^{4} k}{(2 \pi)^{3}} \delta\left(k_{z}-x p_{z}\right) \frac{a^{2} / 4}{\sum_{\mu=1}^{4} \sin ^{2}\left(a\left(p_{\mu}-k_{\mu}\right) / 2\right)} \\
=\frac{1}{4 a} \int_{-\pi}^{\pi} \frac{d^{3} K}{(2 \pi)^{3}}\left[\sin ^{2}\left(\frac{a p_{4}-K_{4}}{2}\right)+\sin ^{2}\left(\frac{K_{1}}{2}\right)\right. \\
\left.+\sin ^{2}\left(\frac{K_{2}}{2}\right)+\sin ^{2}\left(\frac{a(1-x) p_{z}}{2}\right)\right]^{-1} .
\end{array}
$$

The first three terms within diverge as $1 / a$ as a goes to zero. The last term is finite and thus can be calculated to lowest order in $a$ analytically. The coefficient or slope of the ultraviolet divergence can be obtained numerically for finite $a$ by subtracting from $I_{5 L}$ its finite piece. With these steps, we find

$$
I_{5 L}=\left\{\begin{array}{l}
\frac{1}{4 \pi}\left(\frac{n}{a}-p_{z}\right)+\mathcal{O}\left(a p_{z}\right)+\mathcal{O}\left(m^{2} / p_{z}^{2}\right), \quad 0<x<2, \\
\frac{1}{4 \pi}\left(\frac{n}{a}-(1-x) p_{z}\right)+\mathcal{O}\left(a p_{z}\right)+\mathcal{O}\left(m^{2} / p_{z}^{2}\right), \quad x<0 \\
\frac{1}{4 \pi}\left(\frac{n}{a}-(1-x) p_{z}\right)+\mathcal{O}\left(a p_{z}\right)+\mathcal{O}\left(m^{2} / p_{z}^{2}\right), \quad x>2,
\end{array}\right.
$$

where $n / a$ is the radius of a circle which approximates the transverse momentum square (see Figure 3.6). For small $a$ this approximation is arbitrarily exact. Comparing $I_{5 L}$ to its continuum equivalent, we identify the desired correspondence

$$
\Lambda \leftrightarrow \frac{n}{a}
$$

By numerically calculating $I_{5 L}$ we find $n=3.17591$. Note that $n / a$ is larger than $\pi / a$, thus the circle inscribed by the transverse momentum square in $\left(k_{x}, k_{y}\right)$ space (see Figure 3.6) is just slightly larger than shown.

With the UV region correctly analyzed in LPT, we find $\tilde{q}^{(1)}$ in LPT is

$$
\tilde{q}_{\text {Lat. }}^{(1)}=\frac{\alpha_{s} C_{F}}{2 \pi} \begin{cases}\frac{1+x^{2}}{1-x} \ln 2-\frac{1}{(1-x)^{2}}+2+\frac{n}{a p_{z}(1-x)^{2}}, & 0<x<2, \\ \frac{2 x}{1-x} \ln \frac{x-1}{x}-1+\frac{n}{a p_{z}(1-x)^{2}}, & x<0, \\ \frac{2 x}{1-x} \ln \frac{x}{x-1}-1+\frac{n}{a p_{z}(1-x)^{2}}, & x>2 .\end{cases}
$$

The qualitative differences noted between the LPT and Minkowskian continuum one-loop vertex correction can be seen in Figure 3.7 for a given set of parameters chosen as to demonstrate the large effect of an infrared discrepancy.

Despite the success of our initial goal (to extract the form of the UV divergence as it appears in LPT; and thus as it appears on the lattice), the discovery of a discrepancy in the infrared region of the one-loop vertex correcting is troubling at the least. 


\section{$k_{y}$}

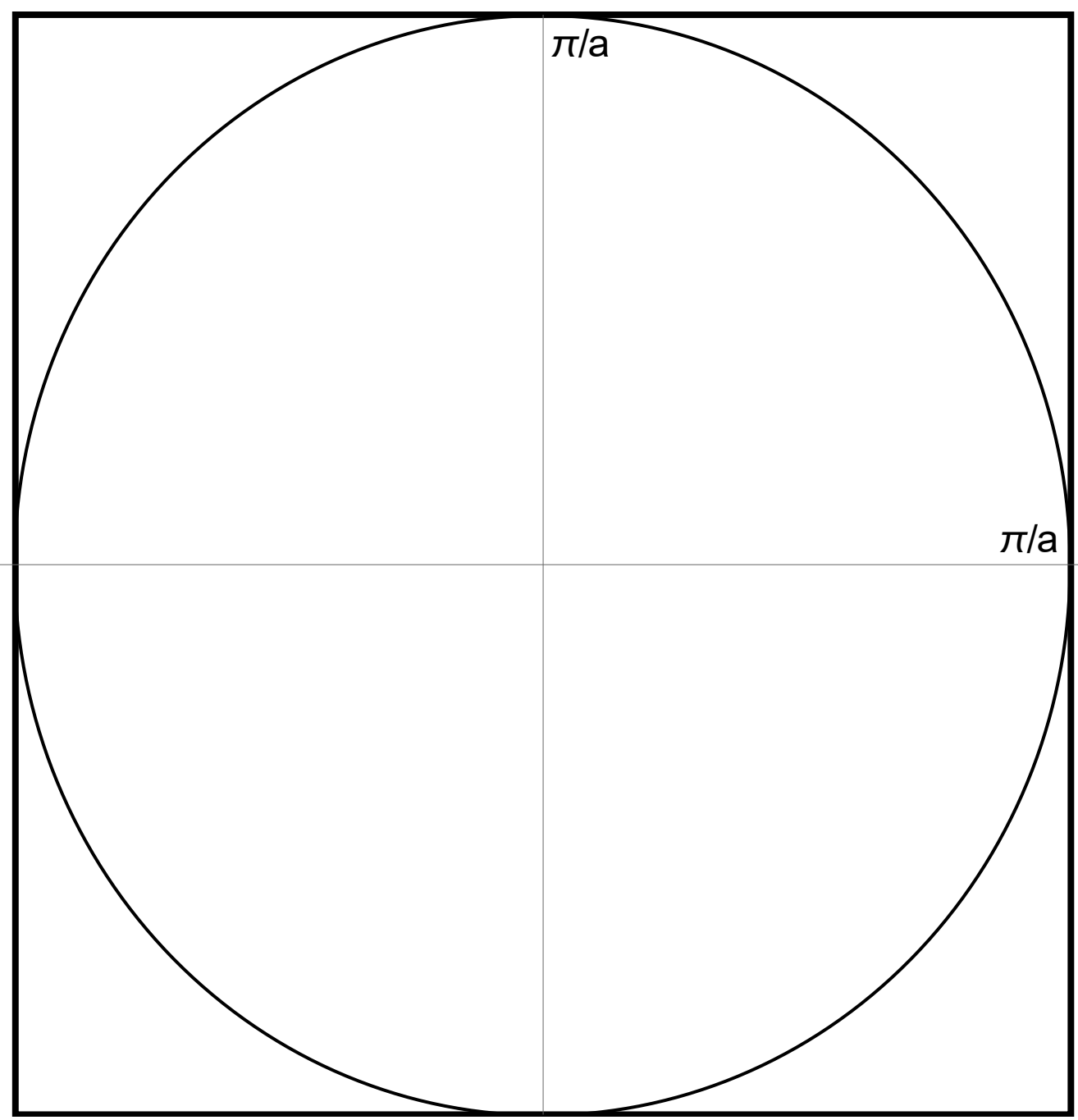

Figure 3.6: The transverse momentum integration region containing the UV divergence in the one-loop vertex correction on the lattice. We can extract the form of the UV divergence by integrating in polar coordinates. The lost terms from the corners will be small for small $a$. Note that the circle inscribed by the rectangle shown is not to scale. To capture the UV divergence correctly, one can show that the circle's radius is $n / a>\pi / a$ where $n=3.17591$ 


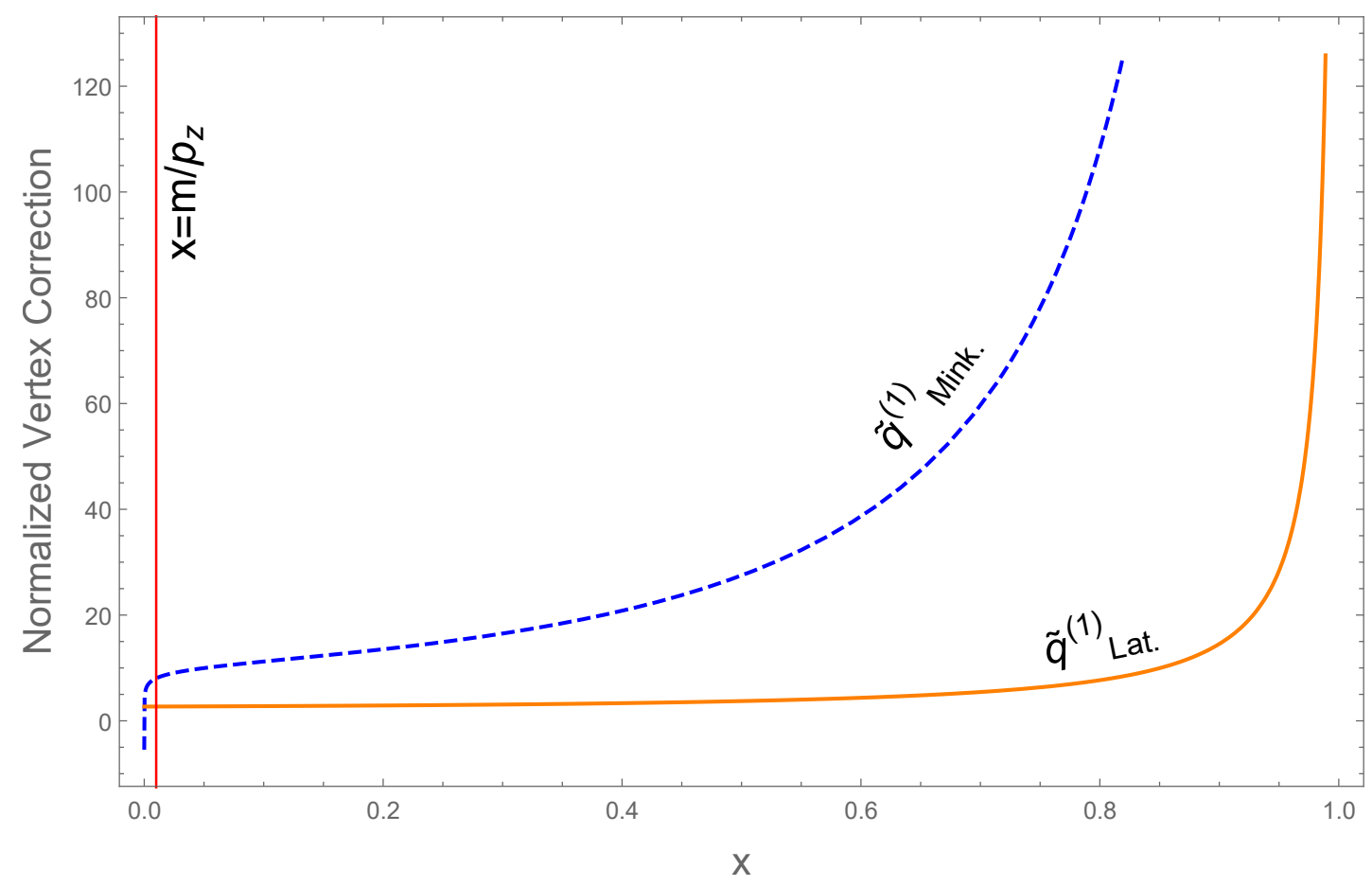

Figure 3.7: The vertex correction to the quark quasidistribution, normalized by $\alpha_{s} C_{F} /(2 \pi)$, calculated in Minkowski space (blue dashed line) versus the same calculated via LPT in Euclidean space (orange solid line) to leading order in $\mathrm{m}^{2} / p_{z}^{2}$ for momentum fraction $x$ between 0 and 1 . Here $p_{z}$ is $2 \mathrm{GeV}$, and the quark mass $m$ is $0.02 \mathrm{GeV} . \Lambda \leftrightarrow n / a$ is $2 \mathrm{GeV}$. When $\Lambda<p_{z}$ the sign of the $x \rightarrow 1$ pole flips for the LPT (orange solid line) result while the Minkoski result remains qualitatively unaffected. The red bar denotes the region where $x=m / p_{z}$. To the left of this line, the expansion in $m^{2} / p_{z}^{2}$ is not valid. 
Furthermore, this discrepancy is independent of the lattice spacing $a$ and thus must be an unexpected result of working in Euclidean space. Regardless of our calculation being perturbative (e.g. at one loop) and the discrepancy being in a region inaccessible to perturbation theory, a difference between Euclidean and Minkowski space within the context of an observable, points to physical differences between the lattice and experiment.

In 3.4, we analyze from where about the infrared discrepancy comes within the oneloop vertex correction integrals. We demonstrate a problem in smoothly rotating from Minkowski to Euclidean space for certain integrals. Taking this at face value, one might jump to the conclusion that the lattice has inherent and subtle issues not yet explored. Our colleagues [reference] have done this exploration. We discuss their enlightening and comforting results in 3.5: the take away, for now, being that the infrared subtlety noticed occurs within lattice perturbation theory and not within the lattice itself.

\subsection{Poles in the Euclidean Integrals}

In Section 3.3 we found a non-UV difference in behavior between the LPT and Minkowskian one-loop vertex correction ((3.86) vs (3.74) respectively). This difference arises from the integral $I_{1}$ of (3.81) encountered within the one-loop vertex correction calculation. Because the difference is not within the UV region, we cannot correct for it perturbatively.

Herein, we analyze in detail the origins of this infrared discrepancy. In doing so, we will find that the Euclidean and Minkowskian versions of $I_{1}$ cannot be Wick rotated into one another without crossing a pole. Thus, they are not the same. One may then immediately jump to the conclusion that the lattice has some rather troubling subtleties. In 3.5 we will discuss recent work from colleagues which point to these subtleties being an artifact of the lattice perturbation theory and not of the lattice itself.

The Minkowskian version of $I_{1}$ in (3.81) is

$$
I_{1 M}=\frac{-i}{(2 \pi)^{3}} \int \frac{d k^{0} d k^{1} d k^{2}}{\left(k^{2}-m^{2}+i \epsilon\right)\left((k-p)^{2}+i \epsilon\right)} .
$$

The Euclidean equivalent is obtained by Wick rotating the energy component of the $k$ 4-momentum,. That is

$$
k_{4}=i k^{0}
$$




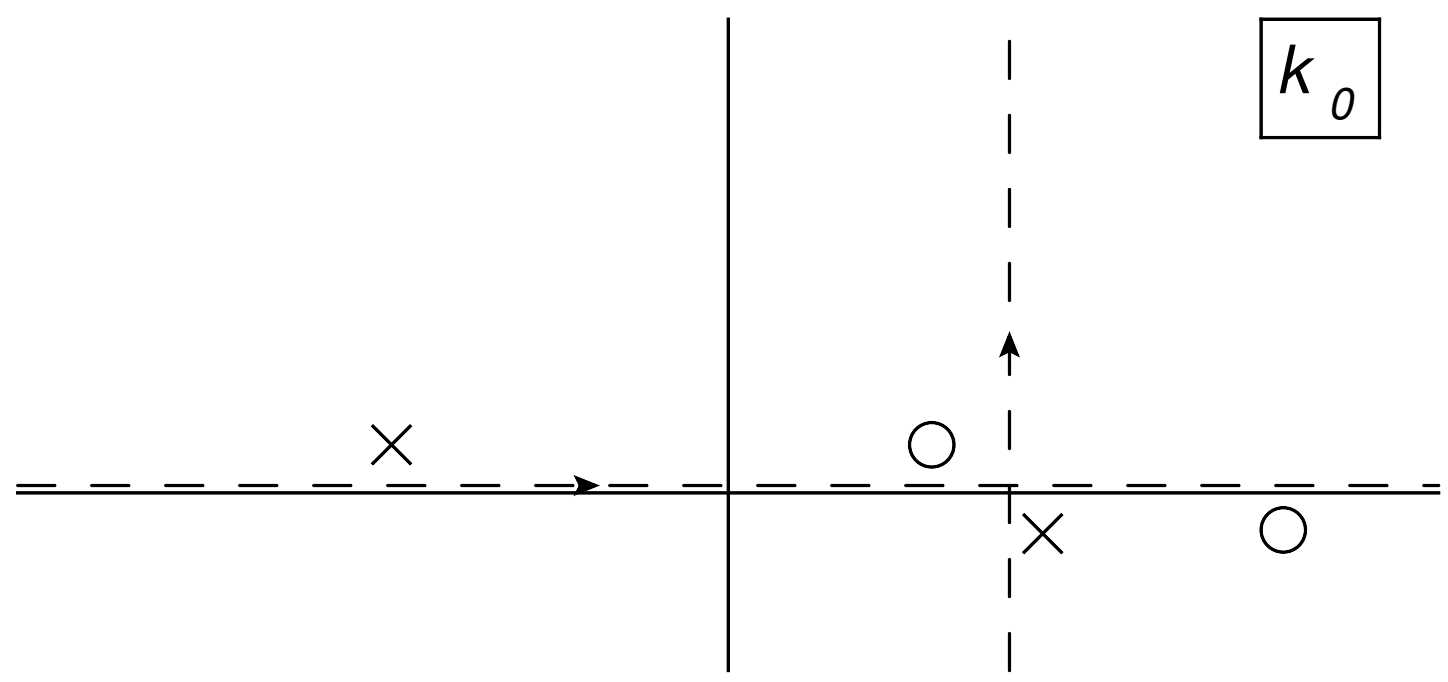

Figure 3.8: Locations of the $k^{0}$ poles within the Minkowskian integral $I_{1 M}$. Crosses represent the two poles arising from the quark propagator, and circles represent the two poles from the gluon propagator. The relative locations of each pole with respect to one another is of importance and is shown accurately within this plot. The vertical dashed line demonstrates a given location of the Wick rotation $k_{4}=i k^{0}$

This transformation is only valid if no poles within $I_{1 M}$ are crossed during the rotation. There are four poles total: two from the quark propagator $1 /\left(k^{2}-m^{2}+i \epsilon\right)$ (denoted $\left.k_{1,2}^{0}\right)$, and two from the gluon propagator $1 /\left((k-p)^{2}+i \epsilon\right)$ denoted $k_{3,4}^{0}$. The locations of these poles are

$$
\begin{aligned}
& k_{1,2}^{0}= \pm \sqrt{k_{\perp}^{2}+x^{2} p_{z}^{2}+m^{2}} \mp i \epsilon \\
& k_{3,4}^{0}=\sqrt{p_{z}^{2}+m^{2}} \pm \sqrt{k_{\perp}^{2}+(1-x)^{2} p_{z}^{2}} \mp i \epsilon .
\end{aligned}
$$

Of much importance is the location of each pole with respect to one another. These locations within the complex $k^{0}$ plane are accurately depicted in 3.8 where the two quark poles are represented by crosses and the two gluon poles by circles. The separation from the real axis is from the $i \epsilon$ imaginary term within the quark and gluon propagators.

One can verify from equation (3.89) within the lower half complex plane of $k^{0}$ the gluon pole is always to the right of the quark pole. And similarly, within the upper half plane, the gluon pole is always to the left of the quark pole. At any given $k_{\perp}=\left(k^{1}, k^{2}\right.$ there will always be particular value of $k^{0}$ for which one can rotate $k^{0}$ into $-i k^{4}$ without crossing a pole (see 3.8 for an example where $k^{0}$ is chosen at a particular real value, say $E_{x}$, between the gluon pole on the upper half plane and the quark pole on the lower half plane. After performing the rotation, we write 3.89 as 


$$
\begin{aligned}
& I_{1 M}=\frac{1}{(2 \pi)^{3}} \int d^{2} k_{\perp} \int_{-\infty+i E_{x}}^{\infty+i E_{x}} d k_{4} \quad \times \\
& \frac{1}{\left(k_{4}^{2}+k_{\perp}^{2}+x^{2} p_{z}^{2}+m^{2}\right)\left(\left(k_{4}-p_{4}\right)^{2}+k_{\perp}^{2}+(1-x)^{2} p_{z}^{2}\right)},
\end{aligned}
$$

where we have made the substitution $k_{z}=x p_{z}$.

This Minkowskian integral is now equivalent to its Euclidean brother $I_{1}$ seen previously in equation (3.81) with the exception of the transposition of the $k_{4}$ integral line by $i E_{x}$. One must simply shift this line onto the real axis to equate $I_{1}$ and $I_{1 M}$ exactly. Here we encounter a difficulty.

As seen in 3.8, the gluon pole in the upper half plane, which we we avoided by choosing $E_{x}$ to the right of it, now precisely lies between the real $k_{4}$ line and $i E_{x}$. We cannot shift $k_{4}$ without crossing this pole. We then ask, in what circumstances will there be a pole between a given $E_{x}$ and the real $k_{4}$ line? Looking at $k^{0}$ in (3.89), we see that the real part of gluon pole in the upper half plane is positive if

$$
k_{\perp}^{2}<p_{z}^{2}-(1-x)^{2} p_{z}^{2}+m^{2}=x(2-x) p_{z}^{2}+m^{2}
$$

This region of $k_{\perp}$ occurs within $I_{1 M}$ (for $0<x<1$, the case of interest here) and therefore we cannot shift $k_{4}$ to equate it to $I_{1}$. Hence, the discrepancy we see in 3.7.

This particular calculation demonstrates why one cannot always, in general, evaluate matrix elements in both Euclidean space and Minkowski space and expect to arive at the same result. Unfortunately, this would appear to suggest a dilemma: the lattice, by necessity, evaluates Euclidean matrix elements whereas we live in a Minkowskian reality. In the following Section 3.5, we discuss work by colleagues which shows how the lattice (though not necessarily LPT!) manages to sidestep and, by design, correct for the issues noted here.

\subsection{The Lattice vs. Lattice Perturbation Theory}

To reiterate, there exists non-trivial differences between the first-order vertex correction to the quark quasidistribution as calculated in Euclidean spacetime (see (3.80)) and in Minkowskian spacetime (see (3.74)). Furthermore, within lattice perturbation theory, a zero-order expansion in the lattice spacing $a$ of the quark quasidistribution will result in the same non-trivial differences, independent of $a$, found with continuum Euclidean result. That is, the behavior of the first-order (in $\alpha_{s}$ ) loop corrections to the quark quasidistribution in Euclidean spacetime is qualitatively different than the behavior of 
the same in Minkowskian spacetime and not an artifact of taking the continuum limit in LPT.

In particular, in Minkowskian spacetime, the first-order loop correction to the quark quasidistribution contains an logarithically infrared divergent term,

$$
\ln \frac{p^{z}}{m^{2}}
$$

which tends towards infinity as the quark mass $m$ is taken to zero. This term is absent in the equivalent Euclidean correction.

This infrared divergence is not a unique feature of our first-order correction to the quark quasidistribution. In fact, previously calculated loop corrections to the physical quark distribution (or general parton distribution functions) contain the same infrared divergent behavior [76, 77]. To be clear, this infrared divergence in not a physical feature of the quark distribution as the infrared regime is not accessible within perturbationbased techniques as $\alpha_{s}$ grows larger than one.

But, an analysis of how perturbation theory breaks down within this regime points to inconsistencies between disparate methods to calculate QCD observables. We should expect two independent methods of calculating a quantity to misbehave in the same manner as we push towards the limit at which the methods themselves breakdown.

If two independent methods did not behave similarly in the infrared limit, then we would have no known way to match the two independent results in a perturbative manner. That is, we would prefer differences which we can correct for to only exist in the non-infrared regime, such that we can apply our matching scheme in an order-byorder systematically through standard and well-known perturbation theory techniques.

Thus, the nonexistence of a logarithmically infrared divergent term in the first-order loop correction within Euclidean spacetime (and thus perhaps in LPT) is disquieting. At the very least, more careful thought would be required in order to understand how to match lattice-based calculations of the quark quasidistribution to the Minkowskian continuum of the same within not just the UV regime, but also, possibly, in the nonperturbative infrared.

In this section, we now discuss a solution to the dilemma outlined above. First, let us recall how, in the previous section 3.4, we carefully analyzed differing behavior between Eucludiean and Minkowskian loop-corrections and, in doing so, arrived at a pole-based argument for why one cannot Wick rotate the vertex-corrections from Euclidean back to Minkowskian spacetime.

There is also a physical argument for this difference in the infrared behaviors which 
provides additional illumination upon our dilemma. Consider, in the Minkowskian vertex correction, one of the encountered integrals over the quark momentum, shown previously in (3.87). One notes that the infrared divergence appears as the quark and gluon's momentum tend towards collinearity. That is, as the quark mass tends to zero, such that the quark and gluon momenta can become more and more parallel where

$$
(k-p)^{2} \rightarrow 0,
$$

leading to a additional divergence in the respective propagators.

But, in the Euclidean case, quark and gluon momenta cannot be oriented in a collinear configuration. Thus there is no such infrared divergence, even when the quark is placed on-shell with zero mass (as analyzed in section 3.3). Furthermore, in Euclidean space, the external quark must have all momenta real. This completely disallows then for the chance of a collinear singularity from the gluon momenta or mass singularity. Therefore, the origin of the infrared behavior in Minkowskian space, and the lack thereof in Euclidean space, is a feature not dependent on the specific choice of external fourmomentum.

With the above considerations in mind, we now take a step back and look at what we're analyzing, when we calculate the quark quasidistribution in Minkowskian and Euclidean spacetime. This line of thought will lead to a solution to the discrepancy first thought out by our collaborators at Jefferson Lab and Rutgers and analyzed further below.

The amplitude squared for the DIS process (3.3), from which we extracted the hadronic tensor (3.9), has the incoming and outgoing constituent quarks as intermediate states of the incoming proton and outgoing hadronic matter. The intermediate quark momenta is then integrated over.

In the late 1970s, Ellis, Georgi, Politzer et al. [81, 82] showed that the leading-order contributions to hadronic processes, codified within the hadronic tensor, occur when the intermediary quarks are on-shell. In fact, it is this observation which allows us to separate and identify the quark distribution function (3.40) from the hadronic tensor.

It may be that this factorization of the leading order contributions to the hadronic tensor depends on whether one is factorizing in Euclidean or Minkowskian spacetime. Furthermore, note that, in a numerical calculation within Euclidean space, the intermediary quarks with all momentum components real cannot be placed on-shell.

This would seem to place some doubt on whether one can apply standard Minkowskian QCD factorization to Euclidean hadronic calculations. Therefore, determining what re- 
places the leading-order contributions to the hadronic tensor may be relevant then in connecting the Euclidean and Minkowskian calculations of the quark (quasi)distribution.

Motivated by the observations above, Raúl Briceño, Chris Monahan, and Maxwell Hanson [60] carried out the Euclidean-equivalent analysis of leading-order contributions to QCD correlation functions. Therein, Briceño et al. found that one may overcome the dilemma within the infrared regime, presented above, by carefully extracting the leading-order contributions from the correlation function, in which the quark (quasi)distribution function exists, where the correlation function is that used by the lattice itself (rather than the standard one defined in Minkowskian QCD perturbation theory).

In particular, the lattice calculates correlation functions of matrix elements which contain initial and final states that are separated over large distances in time (i.e. steady state matrix elements). An example, Minkowskian correlation function of the quark quasidistribution, given in (3.60), is

$$
C_{M}=\int_{-\infty}^{\infty} \frac{d z}{4 \pi} e^{i x z P^{z}} \int d^{4} y \int d^{4} y e^{-i P^{\prime} y^{\prime}} e^{i P y}\left\langle 0\left|\psi\left(y^{\prime}\right) \bar{\psi}(z) \gamma^{z} \psi(0) \psi(y)\right| 0\right\rangle,
$$

where $P$ is the momentum of the initial quark state and $P^{\prime}$ is the momentum if the final quark state. N.B. we've chosen to work in axial gauge where $W(z)=1$. Our steady state condition requires that

$$
\tau^{\prime}-\tau \gg \omega
$$

where $\omega$ represents the energy of any particular interacting particle and where $\tau$ and $\tau^{\prime}$ are the time components of the incoming and outgoing state 4-vectors $y$ and $y^{\prime}$ respectively.

In Minkowskian spacetime, one can use the well-known Lehmann-Symanzik-Zimmermann (LSZ) reduction formula to write the correlation function above, in momentum space, as the quasidistribution multiplied by the on-shell poles of the incoming and outgoing quark states. That is,

$$
C_{M}=\frac{i}{P^{\prime 2}-m^{2}} \int_{-\infty}^{\infty} \frac{d z}{4 \pi} e^{i x z P^{z}}\left\langle P^{\prime}\left|\bar{\psi}(z) \gamma^{z} \psi(0)\right| P\right\rangle \frac{i}{P^{2}-m^{2}},
$$

where $m$ is the quark mass.

And similarly, for the Euclidean spacetime equivalent of our correlation function, we have

$$
C_{E} \sim \frac{1}{P^{\prime 2}+m^{2}} \int_{-\infty}^{\infty} \frac{d z}{4 \pi} e^{i x z P^{z}}\left\langle P^{\prime}\left|\bar{\psi}(z) \gamma^{z} \psi(0)\right| P\right\rangle \frac{1}{P^{2}+m^{2}}
$$


where, now, all 4-vectors are written in Euclidean spacetime. We write the $\sim$ here to denote that the on-shell poles of our Euclidean $P^{2}$ and $P^{\prime 2}$ momentum-squared scalars must be analytically continued from their Minkowskian versions in order to reproduce the pole conditions $P^{2} \rightarrow m^{2}$ and $P^{\prime 2} \rightarrow m^{2}$.

This analytic continuation of the Minkowskian on-shell condition exists as a section of the integrals over incoming and outgoing final state spacetime positions given with the initial definition of the correlation function above. Fortunately, the large-temporal separation condition, which is a necessary condition for correlation functions calculated on the lattice, corresponds with the region of the spacetime integrals which provides the analytic continuation necessary to take $C_{E}$ to $C_{M}$.

That is, the lattice, which calculates $C_{E}$ for large temporal separations, calculates the exact region in which $C_{E} \rightarrow C_{M}$. Thus, though the Minkowskian and Euclidean one-loop contributions to the quark quasidistribution qualitatively differ, their respective correlation functions, for incoming and outgoing quark states separated by long distances in time, do not. Said in another way, the lattice version of the Minkowskian LSZ reduction formula, provides the prescription which analytically continues the Euclidean correlation functions around the troublesome poles encountered in 3.4 and, in doing so, reproduces the Minkowskian correlation function.

We have then a satisfactory answer to the puzzle encountered in 3.3. And the answer is thus: in order to match lattice calculations of distributions perturbatively (as done, for example, in [65]), such as corrections to the quark quasidistribution, one must instead match the correlation functions of the respective distributions over large temporal separations as it is these correlation functions which are directly probed on the lattice. These correlation functions, on the lattice compared to the Minkowskian continuum equivalent, will differ in their scheme-dependence (i.e. ultra-violet regularization), lattice-spacing effects, and finite-volume effects, but not by qualitative discrepancies in the infrared regime. Therefore, at least in the present case analyzed, the one-loop vertex corrections to the quark quasidistribution at zero'th-order in the lattice spcaing $a$ remain the same as those captured by the perturbative UV divergences found in (3.86). 


\section{Chapter 4}

\section{Concluding discussions}

E proceeding work was undergone with two objectives. One, analyze how renormalizibility, or the lack there-of, and high-energy constraints become

intertwined and necessary considerations for BSM additions to the standard model which alleviate the proton radius and muon anomalous magnetic moment discrepancies between theory and experiment. Two, provide the ultraviolet connection between lattice perturbation theory (necessarily in Euclidean spacetime) and experiment (necessarily in Minkowskian spacetime) within the context of one-loop corrections to the distribution of quark momenta within a given hadron. In doing so, we further set to analyze how the Euclidean and Minkowskian spacetime versions of the quark distribution unexpectedly differ in the non-UV region. Motivated by this infrared discrepancy, we also discuss an interesting lattice-based resolution, provided by collaborators - namely, that the lattice itself calculates correlation functions, separated by large distances in time, of these distributions rather than the distributions themselves. And, the correlation functions are demonstratively equivalent whether calculated in Euclidean or Minkowskian spacetime. In Chapter 1, we provided the context for these two objectives by offering a brief two-part respective review of the standard model.

In Section 1.1 of the introduction, we outlined the electroweak sector of the standard model. We included, within this general review, a discussion on the mass-generating Higgs mechanism via spontaneous electroweak symmetry breaking. We additionally provided an example of the well-behaved nature of the theory by considering the famous Gell-Mann Goldberger Kroll Low (GGKL) calculation of the $\nu \bar{\nu} \rightarrow W^{+} W^{-}$process (see Fig. 1.2). The GGKL process, and Weinberg's response to it, provide illumination for the inner workings of renormalizable Yang-Mills theories, which became useful for understanding the context of our discussion on extensions to the electroweak sector in Chapter 2. 
We continued our overview of the standard model with a discussion of the strong sector in Section 1.2. The theory of the strong sector, quantum chromodynamics (QCD), contains a peculiar property known as as asymptotic freedom. That is, the interacting matter of QCD becomes nearly non-interacting and almost free when probed at high energies (above $200 \mathrm{MeV}$ ). Though asymptotic freedom at short distances means QCD is inherently renormalizable and well behaved, the theory at low energies becomes inconvenient for theorists to calculate. Namely, for low energies and long distances, the QCD equivalent of the fine-structure constant grows larger than one and the theory is no longer accessible via standard quantum-field-theory perturbative techniques.

This non-perturbative nature of QCD at low-energies requires then some new theoretical methods to calculate observables. We thus ended Section 1.2 with a brief discussion of a numerical-based technique, Lattice QCD (LQCD). The "lattice" is a computational discretization of Minkowskian spacetime onto a granular Euclidean grid within four dimensions, placed within a finite-volume hypercube. This discretization allows powerful computers to calculate the correlation functions of QCD matrix elements in a non-perturbative manner. However, one must understand how to account and correct for potential artifacts encountered due to this discretization and Euclidean-space rotation. It's this "matching" between the LQCD and experiment which motivated our analysis in Chapter 3.

\subsection{Discussions on the proton radius puzzle}

Within the last 8 , or so, years, experimentalists have consistently found a discrepancy between the proton's electric charge radius (denoted $R_{E}$ and defined in (2.17)) measured by electron-proton interactions and muon-proton interactions. Although some to-bepublished experimental results may place new light upon the proton radius discrepancy, it remains the case that muon-based experiments find the proton's radius to be about $4 \%$ smaller than electron-based results with a 5.6 standard deviation disparity. This high-level of confidence within the analysis results arises from the increased precision of muonic-hydrogen-based experiments due to the increase in relative size of the proton as viewed by the muon (compared to the electronic-hydrogen equivalent analysis). The muon, being orders of magnitude heavier than the electron, orbits the proton orders of magnitude closer than the electron, and thus can comparatively resolve the proton's size at a higher resolution.

Considering that the $5.6 \sigma$ discrepancy is real, in Chapter 2 we set forth to find 
suitable beyond-standard-model theories which would explain the apparent non-zero $\delta R_{E}$ of (2.4). Furthermore, the theory we study can simultaneously to solve the $3.5 \sigma$ similarly ongoing discrepancy between the anomalous magnetic moment for the muon as extracted from experiment and as predicted from theory (see equations (2.5) and (2.6) respectively).

A straightforward method of extending the standard model to solve both the proton radius puzzle and the muon anomalous magnetic moment discrepancy is to add a new neutral vector boson mediator, often denoted $\phi$, to the theory which interacts with the muon differently than it interacts with the electron. This violates so-far well-accepted lepton universality and is heavily restricted by a plethora of constraints [24, 25, 26]. We thus developed our BSM extension to fall within the allowed region of currently known lepton-universality constraints.

Of particular interest to the BSM theories considered was the work of Karshenboim et al., wherein the necessity to consider additional constraints from radiative corrections to the $W$ decay width was shown to be paramount [26]. These radiative corrections, as we point out, are related to the renormalizbility of the theory. Similar to the necessity of including a neutral $Z$ boson to cancel out the high-energy behavior of the Gell-Mann Goldberger Kroll Low (GGKL) process (see (1.58)), we therefore included additional charged-vector bosons, named the "shadow $W$ " and denoted $W_{s}$, along with extra triple-boson Yang-mills interactions, wherein the $\phi$ is a treated similar to the $Z$ boson in standard electroweak theory, that fix the high-energy behavior of BSM solutions to the proton radius puzzle noted in Karshenboim's work.

The BSM addition considered, containing vector and axial vector mediators (denoted $\phi_{V}$ and $\phi_{A}$ respectively with respective coupling strengths denoted $C_{V}$ and $C_{A}$ ) to the standard-model Lagrangian is seen in (2.22). The new interactions change the 2S-2P Lamb shift, as shown in (2.29). A change of $310 \mu \mathrm{eV}$ is necessary to solve the proton radius puzzle. This then fixes the parameters of our coupling strengths. Furthermore, the additional $\phi$ interactions change the muon's anomalous magnetic moment by (2.40); this further fixes the allowed parameters of the discussed BSM theory.

A particularly interesting result of the work detailed in 2 occurs within the changes, compared to a theory with no shadow $W$ s, to the radiative corrections to the $W$ decay 
width (seen in (2.48) and repeated explicitly here),

$$
\begin{aligned}
\Gamma_{W}(W \rightarrow \mu \nu \phi) & =\frac{G_{F} m_{W}^{3}\left[\left(C_{V}^{\mu}\right)^{2}+\left(C_{A}^{\mu}\right)^{2}\right]}{96 \sqrt{2} \pi^{3}} \\
& \times\left\{\log ^{2} \frac{m_{W}^{2}}{m_{\phi}^{2}}-5 \log \frac{m_{W}^{2}}{m_{\phi}^{2}}+\frac{37}{3}-\frac{\pi^{2}}{3}\right\} .
\end{aligned}
$$

Due to the careful addition of the "shadow W", the decay width is now well-behaved in the high-energy limit (i.e. as the mass of $\phi$ is taken to zero). Note, though there is still a BSM constraint, coming from $W$ decay, the constraint no longer impacts the parameter space of our coupling constants necessary to solve the proton radius and muon anomalous magnetic moment discrepancies.

Including also additional lepton-universality constraints from energy splittings within muonic Magnesium and Silicon, we plot the allowed parameter space of our solution in Figure 2.3. As shown, there exists a notable range of values for the coupling constants $C_{V}$ and $C_{A}$ and for the mass of our BSM vector-mediator $m_{V}$ which may alleviate both the proton radius puzzle and the muoon anamlous magnetic moment discrepancy without running into the known constraints from experiment.

We also consider the scalar plus pseudoscalar analogue of our vector plus axial vector theory. We show that this analogue is similarly well-behaved and can also be a solution to the dilemma previously set-forth. We plot the parameter space of the scalar theory, along with its constraints, in Figure 2.9.

Thus, we have shown that carefully-crafted BSM solutions to the proton radius and muon anomalous magnetic moment puzzles remain viable despite non-trivial constraints from lepton-universality and the relatively new constraints from heavy-boson radiative corrections.

\subsection{Discussions on lattice quark quasidistribution}

The rapid development of lattice quantum chromodynamics, a computational algorithm for QCD involving the discretization of Minkowskian spacetime on a Euclideanhypercube grid, within the last decade has brought about notable success at analyzing previously intractable low-energy regimes of standard QCD. In order to make sense of the calculations on the lattice, we must understand the procedure which takes the lattice observables back to their continuum Minkowskian analogues. One segment of this procedure was provided in Chapter 3 wherein we analyzed the necessary matching conditions for a specific quantity, namely the distribution of a given hadron's momentum among its constituent quarks. 
Chapter 3 began with a background on deep inelastic scattering (DIS), a process which allows us to extract internal hadronic information from the scattering of a highenergy electron off a target hadron. From the crosssection of this process, one can define the quark distribution function (see (3.40)), which is the distribution of the respective hadron's momentum among its internal quarks. Unfortunately, for lattice work, a fromfirst-principles calculation of the quark distribution function includes integrations over the time-components of momenta. These integrations prove difficult to analyze directly , within a numerical context, as the time-components are Wick rotated to the imaginary axis in the complex plane, and thus are not numerically sensible.

One solution offered by X. Ji and collaborators (for example [64]) is to take a largemomentum limit of the quark distribution function. At leading order in this limit, one finds an approximately equivalent distribution, named the quark quasidistribution (see (3.60)), wherein only the spatial components of momenta contribute such that the quasidistribution is safely calculable on the lattice. We then set forth to understand how to match the lattice version of the quark quasidistribution at a given order in the lattice spacing $a$.

The lattice spacing $a$ operates as an all-orders ultraviolet regulator. In order to match a lattice result back to its physical equivalent, we must remove the lattice-based UV regulator and replace it with a standard continuum-based renormalization scheme (such as MS bar) as used within experiment. Furthermore, we must assure that the final lattice results, post-fixing of the UV regulation, match the continuum Minkowskian equivalent up to small discretization and finite-volume hypercube effects.

Though quantities such as the quark momentum distribution function are fundamentally non-perturbative (hence the need for the lattice), their behavior in the ultraviolet regime is perturbative. Thus we can use perturbation theory on the lattice action (in this case the Wilson action defined in (3.69)) in order to understand how to correct for the UV regime.

We found the first-order vertex correction, which contains the UV-divergence we'd like to match, for the lattice to be

$$
\tilde{q}_{\text {Lat. }}^{(1)}=\frac{\alpha_{S} C_{F}}{2 \pi} \begin{cases}\frac{1+x^{2}}{1-x} \ln 2-\frac{1}{(1-x)^{2}}+2+\frac{n}{a p_{z}(1-x)^{2}}, & 0<x<2, \\ \frac{2 x}{1-x} \ln \frac{x-1}{x}-1+\frac{n}{a p_{z}(1-x)^{2}}, & x<0, \\ \frac{2 x}{1-x} \ln \frac{x}{x-1}-1+\frac{n}{a p_{z}(1-x)^{2}}, & x>2 .\end{cases}
$$

where $n$ is a computational value, approximately 3.17591, result from integrating in spherical coordinates on a grid-discretized lattice (see Figure 3.6 for details). A full 
analysis of this result is provided around (3.86).

Compared to the similarly calculated continuum Minkowskian equivalent (see (3.74), there is one troubling lack of similarity. Namely, the two distributions appear qualitatively different in the infrared regime. The infrared regime cannot be corrected in a similar manner to the UV-regime for we cannot safely apply perturbative techniques as $\alpha_{s}$ increases. The continuum Euclidean analogue of the lattice result (in (3.80)) similarly contains this infrared discrepancy. A visualization of this problem is provided for in Figure 3.7.

We further analyzed the origin of this non-UV disturbance in Section 3.4. Therein we found the Euclidean and Minkowskian integrations within the quark quasidistribution differ due to the poles within a particular integral encountered (see (3.87)). The poles of this Minkowskian integral interfere with the Wick rotation necessary to take the Minkowskian integral to its Euclidean (and similarly lattice-based) equivalent. This feature is further illustrated in Figure 3.8.

The findings above incentivized work by others and was discussed in detail within Section 3.5. In particular, Briceño et al. [60] analyzed the specific correlation function (see (3.94)) of the quasidistribution as calculated by the lattice itself. The lattice calculates a lattice-based correlation function, which is not just the quark quasidistribution itself but also includes explicit integrations over the incoming and outgoing quark momenta, with large temporal separations between the incoming and outgoing states.

The additional integrations over the external momenta, separated over large distances in time, behave in such a way as to cancel the unique infrared behavior within the Euclidean correlation functions, and simultaneously restore the Minkowskian behavior within the same. Therefore, though the quasidistributions themselves differ in their behavior depending on whether they're calculated in Minkowskian versus Euclidean spacetime, the corresponding correlation functions over large temporal separations do not.

This is a very nice result indeed, in that, as long as one carefully applies lattice perturbation theory techniques to the correlation functions as they're probed on the lattice, the standard perturbative matching techniques setforth in Section 3.2 apply. Therefore, our UV matching provided in (3.86) remains the regularization-correction required to transform the lattice results to their continuum Minkowskian equivalent. 


\subsection{The End}

We've introduced the proton radius problem and the muon anomalous magnetic moment discrepancy as possible signs of lepton-universality violation. We explored this violation by introducing a suitable beyond-the-standard-model theory which simultaneously solves both experimental inconsistencies. And we showed that the parameters in the theory presented can be chosen so as to not contradict any of the considered experimental constraints.

We also introduced the process, deep inelastic scattering, through which experimentalists extract low-energy hadronic information such as the distribution of hadronic momenta across its constituent quarks. We further explored how one can calculate this distribution using non-perturbative techniques, namely lattice quantum chromodynamics. The lattice results contain artifacts due to their intrinsic numerical approximations of a continuum Minkowskian reality. We showed how one can correct for some of these artifacts, within a given context (the quark quasidistribution). In doing so, we explored the careful waters on which one must tread in order to correctly understand, and account for, what the lattice is calculating versus what experimentalists are analyzing.

Having shown the above in its entirety, this thesis is here concluded. 


\section{Bibliography}

[1] M. E. Peskin and D. V. Schroeder, An Introduction to Quantum Field Theory, Addison-Wesley, 1995.

[2] S. Weinberg, Phys. Rev. Lett. 19, 1264 (1967).

[3] S. Weinberg, Phys.Rev.Lett. 27, 1688 (1971).

[4] M. Gell-Mann et al., Phys.Rev. 179, 1518 (1969).

[5] C. H. Llewellyn Smith, Phys.Lett. B46, 233 (1973).

[6] R. Pohl et al., Nature 466, 213 (2010).

[7] J. C. Bernauer, AIP Conf. Proc. 1257, 641 (2010).

[8] A. Antognini et al., Science 339, 417 (2013).

[9] K. Hagiwara et al., J.Phys. G38, 085003 (2011).

[10] F. Jegerlehner and R. Szafron, Eur.Phys.J. C71, 1632 (2011).

[11] M. Davier et al., Eur.Phys.J. C71, 1515 (2011).

[12] T. Aoyama et al., Phys.Rev.Lett. 109, 111808 (2012).

[13] P. J. Mohr, B. N. Taylor, and D. B. Newell, Rev.Mod.Phys. 84, 1527 (2012).

[14] P. J. Mohr, D. B. Newell, and B. N. Taylor, Rev. Mod. Phys. 88, 035009 (2016).

[15] M. Tanabashi et al., Phys. Rev. D98, 030001 (2018).

[16] A. De Rujula, Phys. Lett. B693, 555 (2010).

[17] A. Antognini et al., Annals Phys. 331, 127 (2013).

[18] J. Bailey et al., Nucl. Phys. B150, 1 (1979). 
[19] G. W. Bennett et al., Phys. Rev. Lett. 89, 101804 (2002), [Erratum: Phys. Rev. Lett.89,129903(2002)].

[20] G. W. Bennett et al., Phys. Rev. Lett. 92, 161802 (2004).

[21] G. W. Bennett et al., Phys. Rev. D73, 072003 (2006).

[22] B. L. Roberts, Chin. Phys. C34, 741 (2010).

[23] C. E. Carlson, Prog.Part.Nucl.Phys. 82, 59 (2015).

[24] V. Barger et al., Phys.Rev.Lett. 106, 153001 (2011).

[25] V. Barger et al., Phys.Rev.Lett. 108, 081802 (2012).

[26] S. G. Karshenboim, D. McKeen, and M. Pospelov, Phys.Rev. D90, 073004 (2014).

[27] B. Batell, D. McKeen, and M. Pospelov, Phys.Rev.Lett. 107, 011803 (2011).

[28] D. Tucker-Smith and I. Yavin, Phys.Rev. D83, 101702 (2011).

[29] A. C. Zemach, Phys. Rev. 104, 1771 (1956).

[30] C. E. Carlson and B. C. Rislow, Phys.Rev. D86, 035013 (2012).

[31] M. N. Rosenbluth, Phys. Rev. 79, 615 (1950).

[32] R. Karplus, A. Klein, and J. Schwinger, Phys. Rev. 86, 288 (1952).

[33] M. I. Eides, H. Grotch, and V. A. Shelyuto, Phys. Rept. 342, 63 (2001).

[34] U. D. Jentschura, Eur. Phys. J. D61, 7 (2011).

[35] J. P. Leveille, Nucl. Phys. B137, 63 (1978).

[36] D. McKeen, Annals Phys. 326, 1501 (2011).

[37] J. Beringer et al., Phys. Rev. D86, 010001 (2012).

[38] T. Aaltonen et al., Phys. Rev. Lett. 100, 071801 (2008).

[39] V. M. Abazov et al., Phys. Rev. Lett. 103, 231802 (2009).

[40] I. Beltrami et al., Nucl.Phys. A451, 679 (1986).

[41] M. E. Peskin and T. Takeuchi, Phys.Rev.Lett. 65, 964 (1990).

[42] M. E. Peskin and T. Takeuchi, Phys.Rev. D46, 381 (1992). 
[43] J. Hewett, The Standard model and why we believe it, TASI lectures, 1997.

[44] D. Treille, Review of the standard model, in Electroweak interactions and unified theories. Proceedings, Leptonic Session of the 27th Rencontres de Moriond, Les Arcs, France, March 15-22, 1992, pages 131-172, 1992.

[45] G. Gabrielse et al., Phys. Rev. Lett. 97, 030802 (2006), [Erratum: Phys. Rev. Lett.99,039902(2007)].

[46] G. Aad et al., Phys. Lett. B716, 1 (2012).

[47] S. Chatrchyan et al., Phys. Lett. B716, 30 (2012).

[48] P. W. Higgs, Phys. Rev. Lett. 13, 508 (1964), [,160(1964)].

[49] F. Englert and R. Brout, Phys. Rev. Lett. 13, 321 (1964), [,157(1964)].

[50] A. H. Mueller, editor, PERTURBATIVE QUANTUM CHROMODYNAMICS, volume 5, WSP, Singapore, 1989.

[51] N. Isgur and M. B. Wise, Adv. Ser. Direct. High Energy Phys. 10, 234 (1992).

[52] B. Grinstein, Ann. Rev. Nucl. Part. Sci. 42, 101 (1992).

[53] G. T. Bodwin, E. Braaten, and G. P. Lepage, Rigorous QCD predictions for decays of p wave quarkonia, in The Fermilab Meeting DPF 92. Proceedings, 7th Meeting of the American Physical Society, Division of Particles and Fields, Batavia, USA, November 10-14, 1992. Vol. 1, 2, pages 1063-1066, 1992.

[54] C. W. Bauer et al., Phys. Rev. D63, 114020 (2001).

[55] R. Gupta, Introduction to lattice QCD: Course, in Probing the standard model of particle interactions. Proceedings, Summer School in Theoretical Physics, NATO Advanced Study Institute, 68th session, Les Houches, France, July 28-September 5, 1997. Pt. 1, 2, pages 83-219, 1997.

[56] S. Durr et al., Science 322, 1224 (2008).

[57] C. Aubin et al., Phys. Rev. D70, 114501 (2004).

[58] S. Collins et al., Phys. Rev. D84, 074507 (2011).

[59] R. P. Feynman, Rev. Mod. Phys. 20, 367 (1948). 
[60] R. A. Briceño, M. T. Hansen, and C. J. Monahan, (2017).

[61] J. Collins, Cambridge Monographs on Particle Physics, Nuclear Physics and Cosmology 32 (2011).

[62] R. Hofstadter, F. Bumiller, and M. R. Yearian, Rev. Mod. Phys. 30, 482 (1958).

[63] R. P. Feynman, Phys. Rev. Lett. 23, 1415 (1969).

[64] X. Ji, Phys. Rev. Lett. 110, 262002 (2013).

[65] X. Xiong et al., Phys. Rev. D90, 014051 (2014).

[66] X. Ji, J.-H. Zhang, and Y. Zhao, Phys. Rev. Lett. 111, 112002 (2013).

[67] X. Ji, Sci. China Phys. Mech. Astron. 57, 1407 (2014).

[68] X. Ji and J.-H. Zhang, Phys. Rev. D92, 034006 (2015).

[69] X. Ji et al., Phys. Rev. D92, 014039 (2015).

[70] J.-W. Chen, X. Ji, and J.-H. Zhang, Nucl. Phys. B915, 1 (2017).

[71] X. Ji, J.-H. Zhang, and Y. Zhao, Int. J. Mod. Phys. Conf. Ser. 40, 1660053 (2016).

[72] T. Ishikawa et al., (2016).

[73] K. G. Wilson and J. B. Kogut, Phys. Rept. 12, 75 (1974).

[74] K. G. Wilson, Quantum Chromodynamics on a Lattice, in Cargese Summer Institute: New Developments in Quantum Field Theory and Statistical Mechanics Cargese, France, July 12-31, 1976, page 0143, 1977.

[75] S. Capitani, Phys. Rept. 382, 113 (2003).

[76] D. Amati, R. Petronzio, and G. Veneziano, Nucl. Phys. B146, 29 (1978).

[77] H. D. Politzer, Phys. Lett. B70, 430 (1977).

[78] A. Radyushkin, (2016).

[79] A. V. Radyushkin, (2017).

[80] A. Radyushkin, (2017).

[81] R. K. Ellis et al., Nucl. Phys. B152, 285 (1979).

[82] R. K. Ellis et al., Phys. Lett. B78, 281 (1978). 


\section{VITA}

Michael Freid entered the world in San Francisco on the first of November, 1989. He spoke his first word, good, while eating ice cream. Ice cream remains his favorite desert. Preferring math \& science to spelling, Michael gained an unceasing curiosity for the natural world at an early age. Though Michael flirted with physics throughout high school, he initially set his eyes on computer science when he entered Rensselaer Polytechnic Instute in 2008. After his first semester, he picked up physics as an additional major. In 2011, Michael graduated. The following year, Michael was awarded Jefferson Lab's Theory Research Scholarship and began his $\mathrm{PhD}$ at the College of William \& Mary. Michael started his work under Carl Carlson in 2014 to solve the proton radius puzzle. From 2015 through 2017, he researched how to match lattice calculations to experiment. After receiving his $\mathrm{PhD}$, Michael will continue his role with the startup Scopeworker to transform the way enterprises and their suppliers collaborate. 